\title{
Aligned and Coaxial Nanofibers for Tissue Regeneration
}

Frank E. Block III

\author{
A Dissertation submitted \\ In Partial Fulfillment of the \\ Requirements for the Degree of \\ Doctor of Philosophy in Electrical Engineering
}

University of Virginia

May 2012 
(C) Copyright by

Frank E. Block III

All rights reserved

May 2012 
Abstract

Nanofiber scaffolds have been well-documented for use in tissue engineering. These scaffolds are unique because they have a high surface area to volume ratio and can be specifically designed to mimic the naturally-occurring extracellular matrix, through both nanofiber diameter and alignment. Nanofiber scaffolds can be synthesized to mimic this structure by aligning the fibers through mechanical and electrostatic means. This dissertation is focused on controlling the alignment, fiber diameter, composition, and controlled drug release characteristics of nanofiber scaffolds for optimal tissue regeneration.

Aim 1 - Optimum alignment of nanofiber scaffolds for fibroblast response. Synthetic nanofiber scaffolds could be used to mimic the nanoscale extracellular matrix to speed up post-injury recovery time in nervous, bone, tendon, ligament, muscle, and arterial tissues. Aligned nanofiber scaffolds provide more directional cues for cellular response than random fibers, and are nanostructured similarly to nervous, ligament, muscle, and tendon extracellular matrices. Aligned nanofiber scaffolds can be readily synthesized by electrospinning onto a rotating mandrel. These experiments have yielded fibers with angular deviations that range from 360 degrees and diameters that range from $60 \mathrm{~nm}$ to a few microns. Our first aim is to determine how to produce the nanofiber scaffold that would have the best characteristics for tissue regeneration applications. These characteristics include polymer composition, diameter, and alignment. In studying the production of nanofiber scaffolds, we observed that as nanofiber diameters decrease the fibers become more difficult to align using a mandrel. We will measure the alignment of nanofibers of different diameters and of different polymers. Alignment will be examined for decreasing fiber diameters as well as different types of polymer, such as PLGA, PCL, or PPHOS (see glossary). We will also determine what nanofiber diameter and alignment elicits the best PC12 and Schwann cell growth and alignment.

Aim 2- Characterizing release kinetics for coaxially electrospun fibers. Coaxially electrospun fibers with different polymers in the core and sheath are unique materials, not only because of their high surface area to volume ratio, but also because of their ability to elute encapsulated drugs - for instance growth factors - as the fiber degrades. This drug release, in combination with the micro- and nanotopographic cues provided by electrospun fibers can provide additional signaling factors to alter the proliferation and morphology of cells. The core and 
sheath can elute different drugs, so that one drug can be introduced from the scaffold in the first phase of healing and another drug can be released in a later phase to trigger an optimum cell response.

This improved understanding of the influence of alignment, fiber diameter, composition, and controlled release characteristics of nanofiber scaffolds on model cell systems, such as Schwann and PC12 cells, will enable the optimization of nanofiber scaffolds for tissue engineering applications. 


\section{APPROVAL SHEET}

The dissertation is submitted in partial fulfillment of the

requirements for the degree of

Doctor of Philosophy in Electrical Engineering

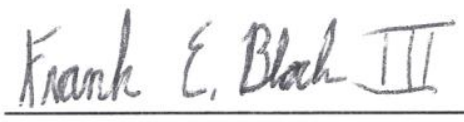

Frank E. Block III, Author

This dissertation has been read and approved by the examining committee:

$$
\text { N. fwomi }
$$

Dr. Nathan S. Swami, Dissertation Advisor
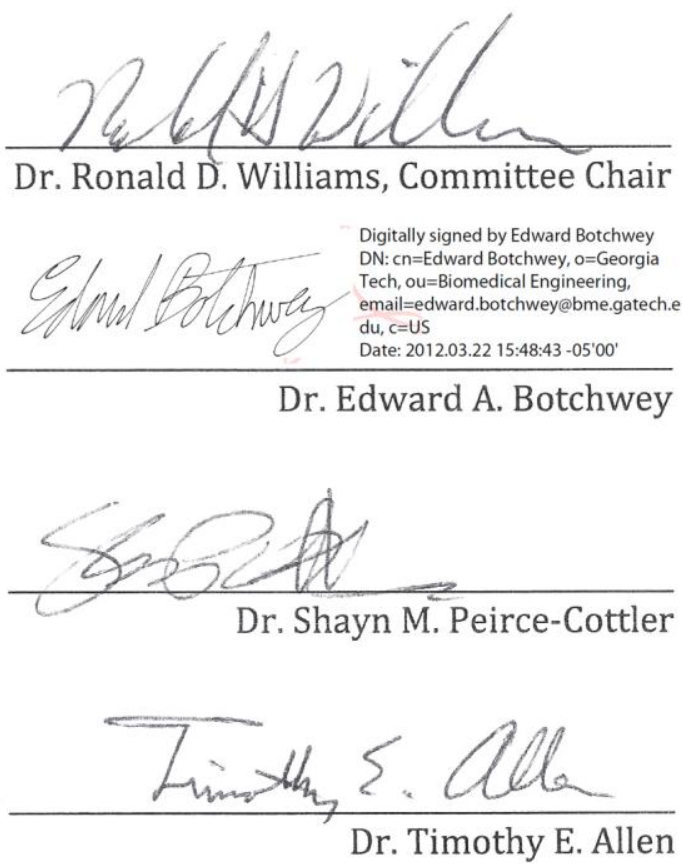

Accepted for the School of Engineering and Applied Science:

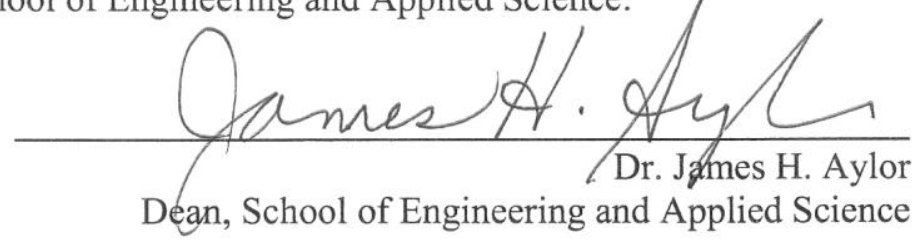

May 2012 
Table of contents

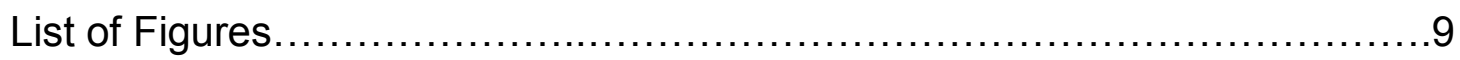

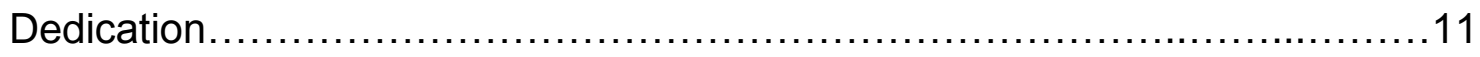

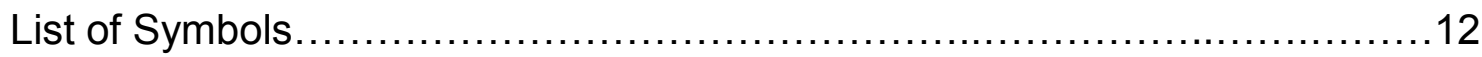

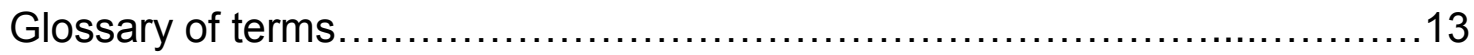

Chapter 1: Introduction...................................................... 16

1.1 Tissue Engineering .................................................

1.2 in vivo aligned tissues............................................ 17

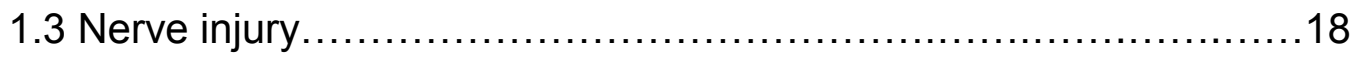

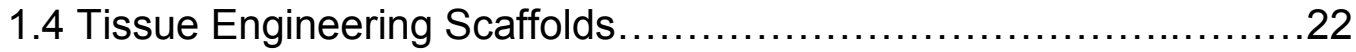

1.5 Scaffold synthesis through electrospinning …....................23

1.6 Unique nanofiber surface area and volume characteristics.........24

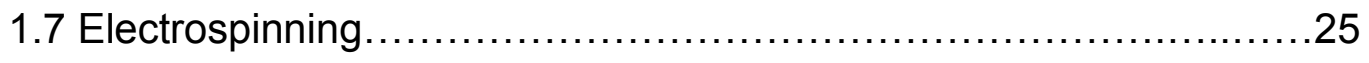

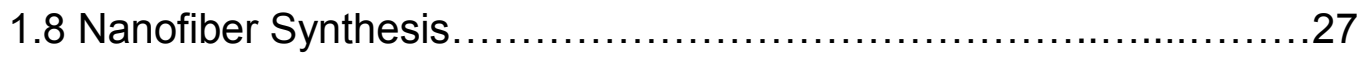

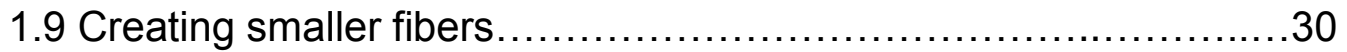

1.10 Cellular interaction with nanofibers................................32

1.11 Current state of the art in small, aligned nanofibers................33

1.12 Conclusions.................................................................

Chapter 2: Aligning small and large nanofibers............................... 35

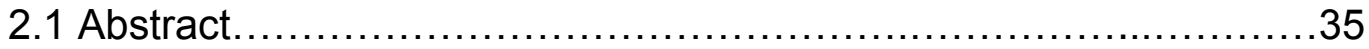

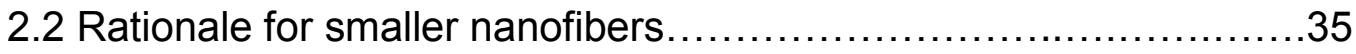

2.3 Creating small aligned nanofibers .................................... 36

2.4 Polymer selection................................................... 41

2.5 Materials and methods for creating successively smaller nanofibers44

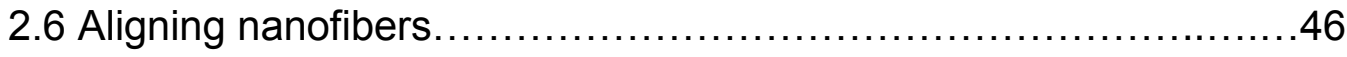

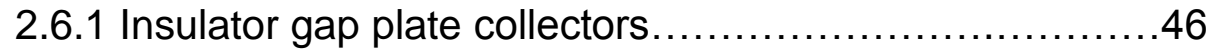

2.6.2 Mandrel collection..........................................48 
2.6.3 Patterned mandrel collection...............................49

2.6.4 Dielectric block collection...................................50

2.7 Varying the residual and stretching electric fields...................51

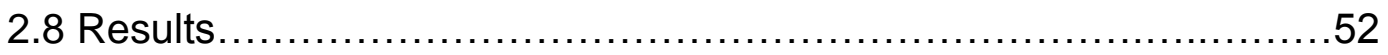

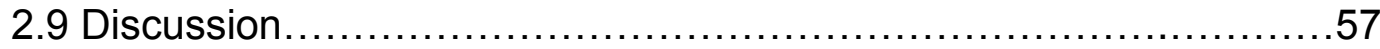

2.9.1 Altering fiber diameter.................................... 57

2.9 .2 Insulator plate gap ........................................ 61

2.9.3 Mandrel and patterned mandrel............................63

2.9.4 Dielectric block and residual charge .........................64

2.10 Effect of magnetic field on nanofibers............................66

2.11 Dielectric alignment of small nanofibers........................67

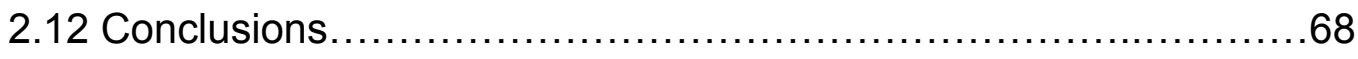

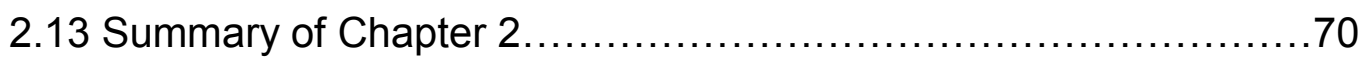

Chapter 3: polymer charge and ease of alignment...........................72

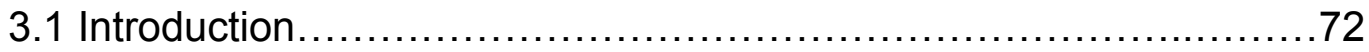

3.2 Materials and Methods ….........................................

3.3 Results and Discussion............................................. 75

3.4 Conclusions.......................................................... 78

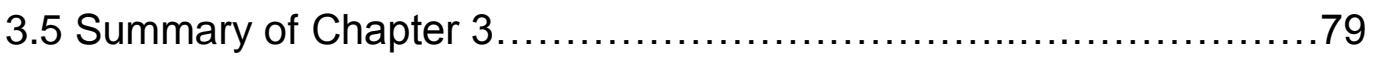

Chapter 4: Enhancing cell adhesion and alignment through the use of aligned PLGA nanofibers ............................................................. 80

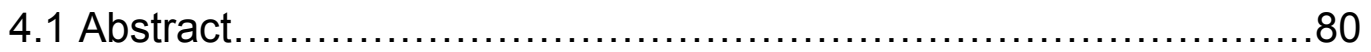

4.2 Fibroblasts on molecularly oriented nanofibers ......................80

4.3 Schwann cell interaction with highly aligned, molecularly oriented

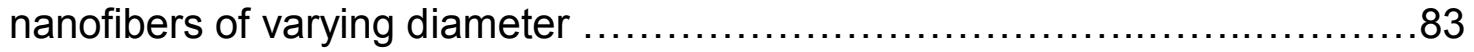

4.3.1 Schwann cell Materials and Methods.......................83

4.3.2 Schwann cell Results and Discussion......................85 
4.4 PC12 cell response to highly aligned sets of PLGA nanofibers.

4.4.1 PC12 cell Materials and Methods. .94

4.4.2 PC12 cell Results and Discussion. .95

4.5 Conclusions. 97

4.6 Summary of Chapter 4 .98

Chapter 5: Coaxial fibers. .99

5.1 Introduction to coaxial fibers. .99

5.1.1 Coaxial electrospinning ...................................100

5.1.2 Characterizing coaxial nanofibers.........................101

5.1.3 Drug delivery with coaxial nanofibers......................103

5.2 Materials and Methods............................................... 105

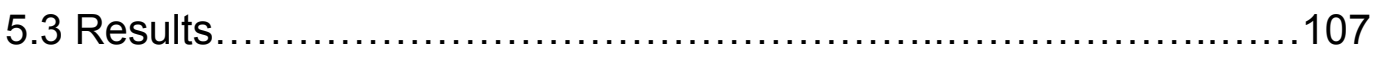

5.4 Discussion.......................................................... 110

5.5 Conclusion....................................................... 111

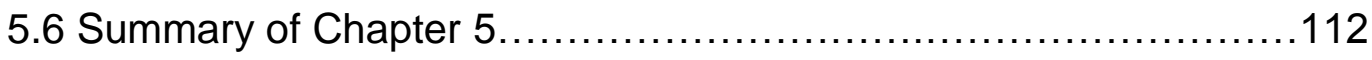

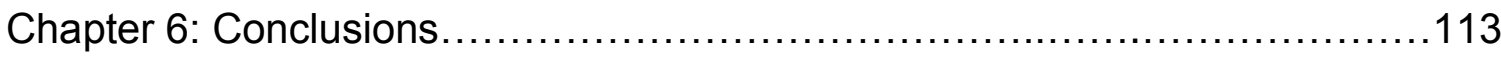

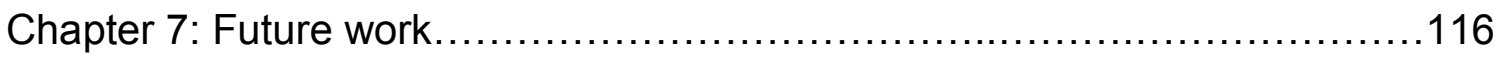

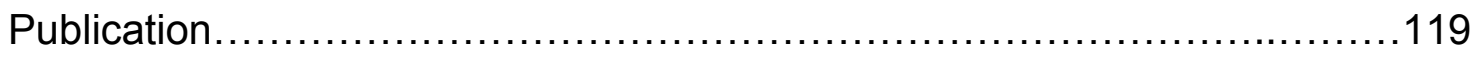

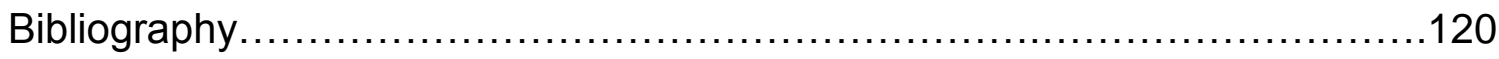

Appendix A: Guide to Polymers and Solvents..............................127

Appendix B: Further information on electrospinning techniques...............130

Section B.1 Nanofiber areas of best alignment........................130

Section B.2 Fiber collection density .................................132

Appendix C: Schwann cell culture, seeding, and staining procedures..........134

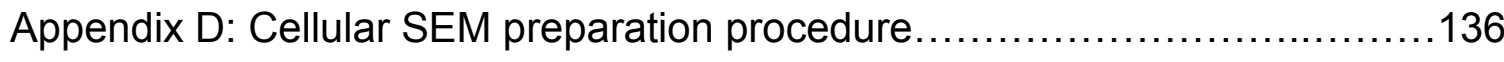

Appendix E: PC12 cell maintenance and seeding procedures................137 


\section{List of Figures}

Figure 1.1: Depiction of axon injury in nervous tissue..........................19

Figure 1.2 The mesoneurium and its components. ...........................20

Figure 1.3: Sunderland's classification of nerve injury. .......................21

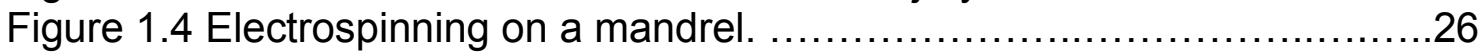

Figure 1.5: examples of aligned and misaligned nanofibers....................30

Figure 2.1: Lack of highly aligned, small nanofibers in the literature............37

Figure 2.2: Electrospinning on a patterned collector...........................38

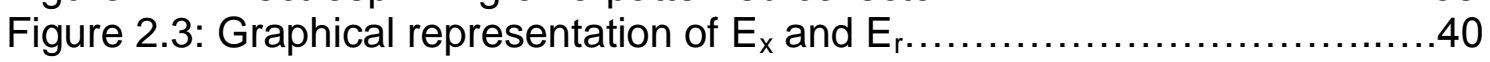

Figure 2.4: poly-glycolic acid (PGA) and poly-lactic acid (PLA) ...............42

Figure 2.5: fiber diameter vs angular deviation for PLGA nanofibers............43

Figure 2.6: A patterned Mylar ${ }^{\mathrm{TM}}$ plate collector..................................47

Figure 2.7: Patterned mylar for patterned mandrel electrospinning ..............50

Table 2.1: Electrospun fiber diameter and angular deviation for mandrel

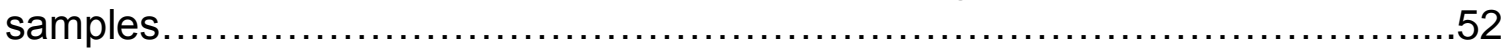

Figure 2.8: Nanofibers grown on gap collectors.............................53

Figure 2.9 Graph of nanofibers' diameter vs. angular deviation................54

Figure 2.10: Nanofibers of varying size grown on dielectric materials.............55

Figure 2.11: Adding TEAC to decrease fiber diameter..........................56

Figure 2.12: Residual charge arches in nanofibers...........................56

Figure 2.13: Relationship between fiber diameter and solution dielectric

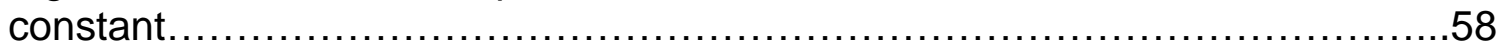

Figure 2.14: PLGA 85:15 fiber diameter vs. solution dielectric strength..........59

Figure 2.15: Comparison between PLGA and blended PN GEG PhPh

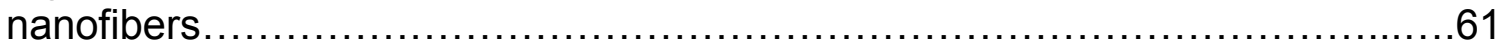

Figure 2.16: Nanofibers on different gap widths.............................62

Figure 2.17: Comparison of fibers on dielectric blocks.......................67

Figure 2.18: Highly aligned nanofibers on dielectric blocks....................68

Figure 2.19: Overview of alignment technique required to align a given size

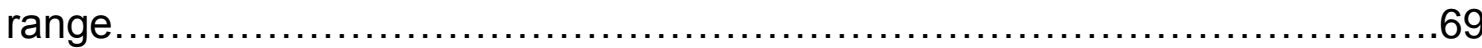

Figure 3.1: PN GEG PhPh, PLGA, and PCL electron fibers on dielectric block collectors.....................................................................

Figure 3.2: Change in residual charges for polymers of different type...........76

Figure 3.3: Nanofibers of different charge on patterned Mylar ${ }^{\mathrm{TM}}$ collectors.......76

Figure 4.1: SEM of fibroblast on large nanofibers..............................82

Figure 4.2: SEM of fibroblast on small nanofibers ............................ 82

Figure 4.3 Schwann cell area on uncoated nanofibers.......................... 85

Figure 4.4: Schwann cell area on polylysine coated nanofibers.................86

Figure 4.5: Schwann cells arranged as bands of Büngner......................87

Figure 4.6: Schwann cells on large mandrel aligned nanofibers.................88

Figure 4.7: Schwann cells on large polylysine coated

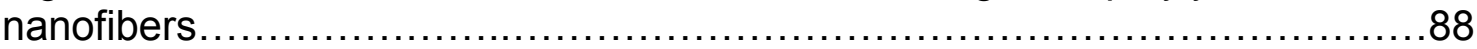


Figure 4.8: 20X magnification image of Schwann cells on nanofibers...........90

Figure 4.9: Bands of Büngner................................................91

Figure 4.10: 40X magnification image of Schwann cells on nanofibers...........92

Figure 4.11: PC12 angular deviation on aligned nanofibers.....................95

Figure 4.12: SEM of PC12 cells on aligned nanofibers..........................96

Figure 4.13: SEM of PC12 cells on small aligned nanofibers...................96

Figure 5.1: Coaxial electrospinning setup ................................100

Figure 5.2: Voltage effect on Taylor cone in coaxial electrospinning............101

Figure 5.3: fluorescent particles in PCL core/sheath coaxial fibers..............102

Figure 5.4: Coaxial release profile of two drugs...............................103

Figure 5.5: PLGA 85:15 coaxial electrospinning parameters...................105

Figure 5.6: PLGA 50:50 coaxial electrospinning parameters..................105

Figure 5.5: Coaxial electrospinning parameters for dual drug fibers...........106

Figure 5.8: PLGA 85:15 core and PLGA 50:50 sheath coaxial fibers...........107

Figure 5.9: PLGA 50:50 coaxial core and sheath fibers.......................108

Figure 5.10: PLGA :15 Inulin-FITC core fibers and release profile..............109

Figure 5.11: Dual drug release profile from coaxial fibers ......................109

Figure 6.1: Advances in the state of the art................................115

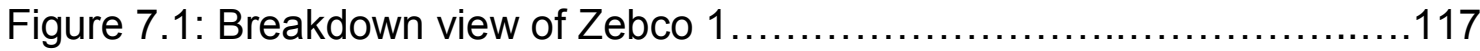




\section{Dedication}

This dissertation is dedicated to my wife, Stephanie, who tolerates my geeky behavior. 


\section{List of symbols}

$d$ - diameter

$E_{r}$ - repulsive alignment field from aligned nanofibers on patterned collectors

$E_{x}-$ stretching electric field experienced by a nanofiber in flight

$\varepsilon_{r}$ - dielectric constant experienced by materials in response to a constant electric field.

I - length

$r$ - radius

SA - surface area

V-volume 


\section{Glossary of terms}

Angular Deviation (AD) - the degree of alignment or misalignment in a nanofiber sample. An angular deviation of zero is a set of perfectly aligned fibers. An angular deviation of $30^{\circ}$ or more is considered poorly aligned fibers. Angular deviation is determined first by calculating the angle of travel of each nanofiber. Then the standard deviation of all of the angles is tabulated.

Axon - a long projection of a nerve cell. Carries nerve signals as action potentials throughout the body.

Biocompatible - a material which is non-toxic and which does not trigger an immune response in the human body.

Biodegradable - a material which breaks down when exposed to conditions found in the body.

Confluence - a term used to measure the density of cells in a Petri dish. At $100 \%$ confluence the cells occupy $100 \%$ of the surface area of the Petri dish.

di-methyl-formamide (DMF) - A common, polar organic solvent which contains nitrogen and two methyl groups.

Dil - a common labeling dye which absorbs light in the green spectra and fluoresces in the orange.

DiO - a common labeling dye which absorbs light in the blue spectra and fluoresces in the green.

Dulbecco's Modified Eagle Medium (DMEM) - a common cell growth medium that contains vitamins and amino acids as well as glucose and essential salts.

Evaporation - (1) a common method of depositing thin films on a material under vacuum by evaporating a solid metal into a gas and allowing the metal to deposit on the target. (2) the process by which a liquid turns into a gas.

f-actin - an important component of a cell's cytoskeleton 
Fetal Bovine Serum (FBS) - a very common cell growth culture additive which contains various proteins and growth factors.

Hexafluoro-2-propanol (HFIP) - an organic, polar, water soluble alcohol commonly used in electrospinning.

High-performance Liquid Chromatography (HPLC) - a chromatographic technique which can determine concentrations of a material in solution.

Hydrolysis - The breakdown of a molecule by the addition of a water molecule. One part of the chemical gains a hydrogen ion $\left(\mathrm{H}^{+}\right)$and the other part gains the hydroxide ion $\left(\mathrm{OH}^{-}\right)$. Hydrolysis is a common means of breakdown of polymers. Hydrolysis can be promoted by acidic or alkaline media, by enzymes, etc.

Laminin - a cross-shaped $(\dagger)$ protein found in the extracellular matrix in almost all tissue.

Mask - an opaque plate which patterns incident light.

Nanofiber (NF) - any fiber with dimensions less than 1000 nanometers.

Nanometer (nm) - 1/ 1000000000 or one billionth of a meter, or one thousandth of a micrometer

Nanofiber coverage - percentage of the area of a sample covered by nanofibers. Higher coverage indicates more nanofibers in the sample, similar to confluence.

Neurite - a projection from a nerve cell body, typically growing into an axon or dendrite.

Pen-strep - a solution of penicillin and streptomycin, common antibiotics for fighting Gram positive and negative bacteria.

Scanning Electron Microscopy (SEM) - a microcopy technique which uses an electron beam to determine characteristics of the surface of a material. These images typically have a magnification of $10000 \mathrm{X}$ or greater.

Schwann cell - a type of cell in the peripheral nervous system responsible for creating myelin, an insulating substance, along the axon of a peripheral nerve cell. 
Sputter coating - a common technique for coating a material with a very thin layer of metal, typically to make it conducting as required for SEM characterization.

Surfactant - a compound which lowers the surface tension of a liquid.

tetra-ethyl-ammonium chloride (TEAC) - an organic, hygroscopic salt.

tetra-hydro-furan (THF) - a common, ring shaped, water-miscible organic solvent.

Tissue Culture Polystyrene (TCPS) - a type of plastic commonly used in Petri dishes.

Transmission Electron Microscopy (TEM) - a microscopy technique which passes an electron beam through a very thin sample to determine its structure. 


\section{Chapter 1: Introduction}

\subsection{Tissue Engineering}

As Arthur C. Clark stated in 1962, "Any sufficiently advanced technology is indistinguishable from magic." [1]. The ultimate goal of Tissue Engineering is to quickly repair damaged tissues in the human body. Tissue Engineering was first reported in the book of Genesis: "So the LORD God caused the man to fall into a deep sleep, and while he was asleep, he took part of the man's rib, and closed up the place with flesh..." [2,3]. Tissue engineering is necessarily a multidisciplinary field that incorporates biology, medicine, materials science, and engineering. The term "Tissue Engineering" was not coined until the mid 1980's when Y. C. Fung proposed to establish a center for living tissues [4]. Researchers around that time had just published a case in which a synthetic polymer was used as a corneal replacement [5].

Thus, Tissue Engineering grew out of the field of biomaterials into its own widely studied discipline [6]. The discipline aims to directly assist damaged body tissues with the regeneration process. When a tissue is damaged, after the initial clotting cascade takes place, vasoconstriction (narrowing of blood vessels with concomitant decreased blood flow) followed by vasodilation takes place. Platelets release growth factors and attract neutrophils and macrophages to clear the site of any foreign debris. Soon thereafter, fibroblasts enter the site and begin producing collagen type III and triggering angiogenesis (the growth of new blood 
vessels). Collagen type III is replaced by collagen type I as the wound healing process concludes [7]. Collagen type III is immature collagen that can be manufactured quickly by young fibroblast cells. Collagen type I is the normal, mature collagen present throughout the body. The collagen type I scaffold forms the basis of the Extra-Cellular Matrix (ECM) which is needed for tissue regeneration. If the wound is too large, surgeons can use skin grafts and other closure techniques to serve as an artificial ECM. Certain tissues regenerate very slowly, or cannot regenerate at all after birth.

\section{2 in vivo aligned tissues}

Certain types of tissue in the human body, such as spinal cord, peripheral nervous, and ligamentous tissues, have highly ordered, aligned ECMs. These tissues are more fragile than others because when they are damaged they either regenerate very slowly or not at all. Ligamentous tissue is used by the body to provide support between bones. It is composed primarily of collagen fibers. Ligament tissue is aligned to help distribute a load along both its length and the connected bones. Too much load at a single point can result in a torn ligament. In the case of ligament tissue in the leg, aligned collagen allows the load from walking to be distributed along the length of the fiber. After a ligament is torn, scar tissue forms and disrupts the overall alignment of the tissue. This newlyformed scar tissue is weaker than the surrounding aligned tissue. If the torn ligament were reattached with an aligned scaffold, the tissue would heal more 
quickly and have better mechanical properties [8]. Some torn ligaments must be sutured together in order for them to heal properly. This surgery can result in additional injury because of overstretching the two ends of the ligament. In such cases, an interposed aligned nanofiber structure would mimic the original tissue before it was injured. An aligned nanostructure will work better than a random nanofiber structure because of the better mechanical properties of aligned fibers along the axis of alignment. A random nanostructure would more closely mimic scar tissue. After an aligned nanostructure is implanted, ligament cells could then infiltrate the scaffold with the goal of reducing the amount of scar tissue in the healed ligament. Nervous tissue also heals better when provided with an aligned scaffold [9-11]. It has also been shown that many other cell types send out processes and otherwise grow and divide in such a way as to align themselves with aligned nanofibers [9].

\subsection{Nerve injury}

Peripheral nerves are a prime example of a tissue that cannot regenerate properly without outside aid. When the axon of a peripheral nerve cell is crushed or severed, the axon and Myelin sheath begin to degrade towards the terminus of the axon. This phenomenon, known as Wallerian degeneration, is depicted in Figure 1.1. Injuries such as this typically require surgical repair. 


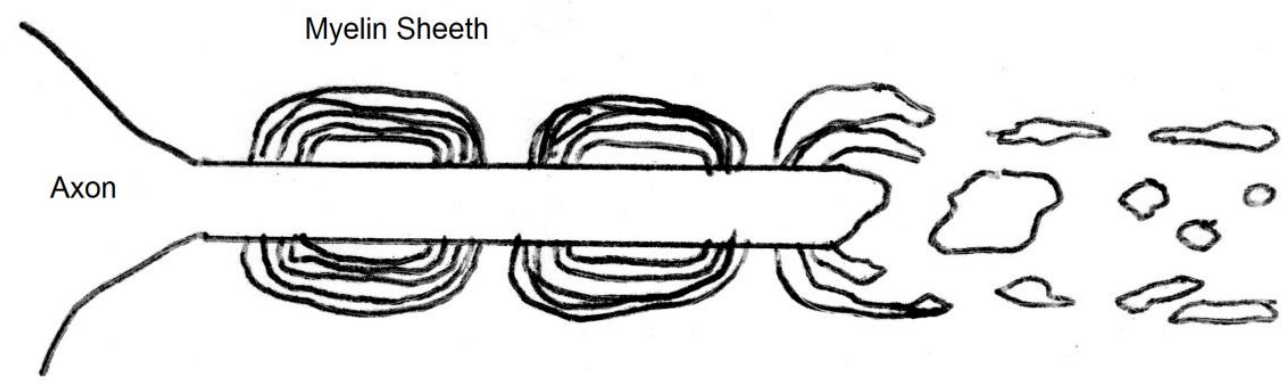

Figure 1.1 depiction of axon injury in nervous tissue. Note the crush injury indicated and breakdown of Schwann cells to the right of the injury. The diameter of the axon is approximately 10 microns [12].

Axons and their basal lamina, the thin outermost layer of a cell, are grouped together in bunches in the perineurium, several of which compose the mesoneurium, a large group of motor and sensory neurons. The perineurium is a cylindrical enclosure composed protects the endoneurium and myelinated axons. The endoneurium is made primarily of collagen I and III strands of $30-65 \mathrm{~nm}$ in diameter and serves as a cushion to protect the myelinated axons [13-15]. The anatomical structure of the mesoneurium and related tissues is depicted in Figure 1.2. 


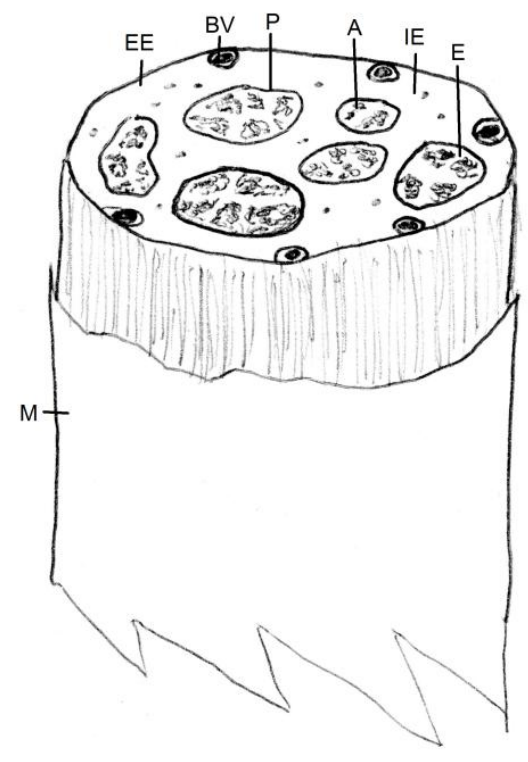

\begin{tabular}{|l|l|}
\hline$A$ & axon \\
\hline BV & blood vessel \\
\hline$E$ & endoneurium \\
\hline EE & external epineurium \\
\hline IE & internal epineurium \\
\hline M & mesoneurium \\
\hline $\mathrm{P}$ & perineurium \\
\hline
\end{tabular}

Figure 1.2 The mesoneurium (M) and its interior components in the peripheral nervous system. A axon, BV blood vessel, E endoneurium, EE external epineurium, IE internal epineurium, M mesoneurium, $\mathrm{P}$ perineurium [15].

Axonal injury can be further classified by Sunderland's classification system, where depending on what part of the nervous tissue is intact (i.e. axon, endoneurium, perineurium, etc.) the spontaneous recovery time can be approximated, as shown in Figure 1.3. 


\begin{tabular}{|l|l|l|l|l|l|l|}
\hline Type & Axon & $\begin{array}{l}\text { Basal } \\
\text { lamina }\end{array}$ & Endoneurim & Perineruim & Epineurium & Recovery \\
\hline I (Neuropraxia) & Intact & Intact & Intact & Intact & Intact & hours \\
\hline II (Axonotemesis) & Disrupted & Intact & Intact & Intact & Intact & 3 months \\
\hline III & Disrupted & Disrupted & Disrupted & Intact & Intact & $\begin{array}{l}\text { Incomplete } \\
\sim 3 \text { months }\end{array}$ \\
\hline IV & Disrupted & Disrupted & Disrupted & Disrupted & Intact & None \\
\hline V (Neurotemesis) & Disrupted & Disrupted & Disrupted & Disrupted & Disrupted & None \\
\hline
\end{tabular}

Figure 1.3: Sunderland's classification of nerve injury [16].

From Figure 1.3 it is obvious intervention is required to promote recover, especially in injury types III - V where incomplete or no recovery occurs. Surgeons can currently address these recovery problems by one of several techniques: suturing together the two severed ends of the mesoneurium, implanting an allograft from cadaver tissue, implanting an autograft from a lessimportant patient nerve, or implanting a nerve guidance conduit. Each technique has an associated set of drawbacks. Suturing together the two ends of the mesoneurium is perhaps the simplest fix, but this may result in further nerve damage as the nerve is often stretched and must grow along the mesoneurium to reinnervate their target $[13,16]$. Allografts are not always available and are prone to infection. They also run the risk of being rejected by the recipient's immune system. Autografts can be less than ideal because a second incision is required and the patient may experience pain or numbness from the harvested nerve. Nerve guidance conduits can be expensive to manufacture, but recent 
developments in the field of tissue engineering could make these scaffolds the preferred form of treatment.

\subsection{Tissue engineering scaffolds}

The goals of a tissue engineered scaffold are multiple. The scaffold must provide structural support for cells to grow and repair the wound. To do so, the scaffold material must not only be highly biocompatible, but also porous enough to allow cells a few microns across to infiltrate the scaffold and thus live inside the scaffold. The scaffold itself must also have similar mechanical properties to the surrounding tissue to prevent further injury, and the size and shape of the scaffold should be adjustable and adaptable. The long-term properties of the scaffold must also be considered: whether the scaffold is to be permanent, such as a stent in an artery, or if the scaffold is to degrade, such as certain types of sutures. If the scaffold degrades, its degradation products must be non-toxic and non-bioactive so that the body can remove and excrete the degradation products [16-17]. The many scaffolds currently under development in tissue engineering are made of polymers. Polymers are an ideal material as many are resorbable, biocompatible, and have tailorable mechanical strength [18]. Additional information on different polymers that can be used in scaffolds is located in Appendix A. Polymer degradation is discussed in Chapters 2 and 5. 


\subsection{Scaffold synthesis through electrospinning}

Scaffolds can be created from many biocompatible polymers. Most scaffolds have a cross sectional area of interest of at least $1 \mathrm{~cm}^{2}$ and a variable height on the order of a few microns to hundreds of microns. A relatively high throughput is required of the fabrication technique to achieve the desired scaffold dimensional volume. Other factors to consider are the focal adhesion sites for the cells, a property closely related to how textured ("rough" or "smooth") a scaffold is, and the macroscopic material properties of the scaffold such as its rigidity and flexibility $[17,19]$. Cells prefer a rougher scaffold onto which protein can more easily adsorb. A cell's focal adhesion complexes are typically on the order of a square micron, and are used to anchor the cell to the ECM. To successfully synthesize a scaffold with appropriate dimensions for cellular integration, one may choose one of two approaches: top-down or bottom-up. A top-down approach involves taking a bulk material and patterning it on the nanoscale such as gas-foaming a material, etching a material with an electron beam, microprinting, or electrospinning. Gas foaming materials yields highly micro-textured, porous, rigid foam scaffolds [20]. Etching a material with an electron beam provides precise control when patterning a material on a nanoscale, but patterning large areas is cost- and time-prohibitive, and one can only write one line at a time with an electron beam, making it very difficult to construct elaborate 3D structures [19]. Micro-printing a material uses a polymer solution and can "print" dots of material down to a few microns, similar to the way that an ink-jet printer works on the macro scale. This micro-printing technique works only for 
certain materials, and provides poor control of 3D scaffolds. Electrospinning requires a polymer solution and can produce either nanofibers or nanobeads with very high throughput [21].

On the other hand, a bottom-up approach involves creating small molecules and allowing the molecules to self-assemble into a scaffold, for instance, the way that microtubules are formed. Bottom-up approaches are difficult because they rely on association kinetics in chemical reactions which are usually very low yield [22]. Of all these available techniques, only electrospinning yields a large (many square centimeter), thick mesh of nanofibers which closely resemble the collagen matrix of the ECM.

\subsection{Unique nanofiber surface area and volume characteristics}

As fiber diameter decreases, the volume decreases more quickly than the surface area. These can be approximated by the standard geometry formulae for volume and surface area of a cylinder where $\mathrm{V}=$ volume, $\mathrm{SA}=$ surface area, $r=$ radius, and $\mathrm{I}=$ length. The end caps of the cylinders are not important because nanofibers collect as unbroken, continuous fibers [18].

Eq. 1.1

$$
V=\pi r^{2} l
$$

Eq. 1.2

$S A=2 \pi r l$ 
For a characteristic length, $l$, as $r$ decreases the volume decreases more rapidly than the surface area. If $r$ decreases to $1 / 2$ of the initial value, then the surface area decreases to $1 / 2$, but the volume decreases $1 / 4$ of initial. Once the fibers reach the nanometer scale, they have very interesting properties because the ratio of the surface area to volume is very large.

\subsection{Electrospinning}

One way to create nanofibers is to use an electrostatic field to "spin" the fibers onto a collector, much in the way that one would spin thread from wool on a macro scale. Electrospinning is a process which yields many similar nanoscale fibers, requiring only a "spinnable" solution and a high voltage source. A spinnable polymer solution can be readily made from any of a number of polymers in an organic solvent such as chloroform or in an inorganic solvent such as water. Current theory about solvent action in polymers is that the solvent enters between the polymer chains to allow motion between the polymer molecules [23]. This interaction allows the polymers dissolve in the solution. This solution is then loaded into a syringe and the syringe is placed into a syringe pump. 


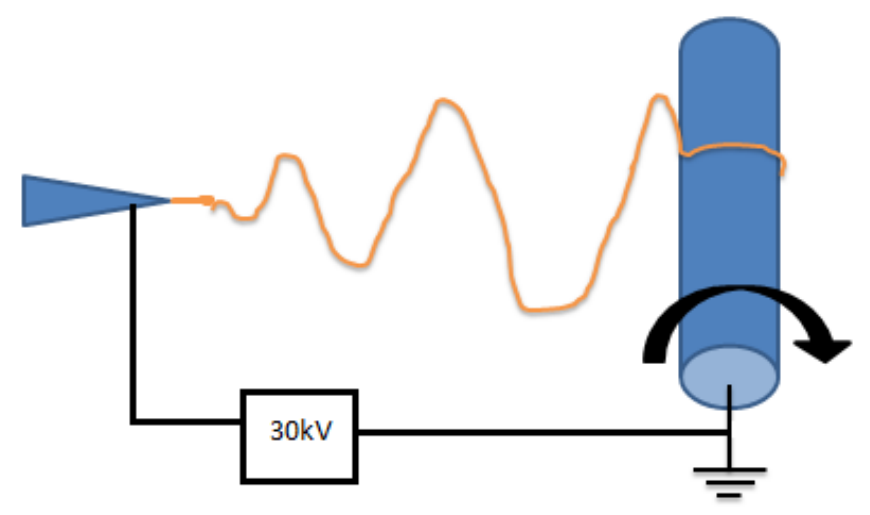

Figure 1.4: Electrospinning on a mandrel. Liquid is pumped out of the syringe (blue triangle) towards the rotating mandrel. Nanofibers, shown here as an orange line, form after undergoing a looping and whipping instability region before they collect on the rotating mandrel. Typically the mandrel is wrapped with aluminum foil so that it will conduct electricity.

A "spinnable" solution must be electrostatically polarizable and must have enough surface tension to form droplets when pumped out of the syringe. Electrostatically polarizable means that an applied electric field causes the solvent molecules in the solution to line up positive to negative, an event which allows the solution to stretch into a nanofiber during the time that an electric field is applied to the syringe. When the high voltage of this electric field is applied to the polymer solution ejected by the syringe, the solution is stretched from a coneshaped droplet at the end of a conducting needle. This stretched droplet is called a Taylor cone, named after Sir Geoffrey Ingram Taylor [24]. At the tip of the Taylor cone, the polymer undergoes stretching in an instability region, where the solution is greatly stretched as like charges repel each other and the solvent evaporates. This stretching allows the formation of a continuous micro- or nanofiber. Nanofiber "coverage" or number of fibers per unit area is very difficult 
to control. Only one publication aims to directly analyze fiber density, and found that increased fiber density correlates with increased cell attachment [25]. Fiber density is further discussed in Appendix B. Microspheres, also called microbeads, can be created in the electrospinning process when the voltage, flow rate, needle diameter, or solution viscosity parameters are not optimized [18]. Typically in these cases the polymer solution viscosity is too high.

\subsection{Nanofiber Synthesis}

A variety of polymers have been electrospun into nanofibers [26-28]. Some of the most commonly used fibers for electrospinning are PLGA, PCL, PVA, PAN, PPHOS, and PEO. Common polymers used in nanofiber synthesis are described in Appendix A. Using electrospinning, one can also create biologically active nanofibers by loading the solution with materials such as collagen (collagen alone is difficult to electrospin) [29-31], or by mixing unspinnable polymers with spinnable ones. Polymers are uniquely advantageous in creating biological scaffolds, because their biocompatibility as well as their degradation properties can be tailored to specific needs. For instance, rigid polymers such as PPHOS can be used in bone scaffolds [32], and less rigid polymers such as PCL can be used in tendon scaffolds. The degradation of a polymer can also be altered through blending a slowly degrading polymer with one that degrades more quickly. Another way of tailoring polymer degradation can be to change the molecular groups on the polymer backbone. 
Polymers with alternating units in the polymer chain, that is, a co-polymer, can be modified to give desired degradation times. These units can repeat in a pattern or randomly along the backbone of the polymer. The best example of a degradable copolymer is PLGA, in which the ratio of lactic acid to glycolic acid can be varied. Lactic acid in PLGA has an extra methyl-group which glycolic acid lacks, and thus the extra methyl group makes lactic acid more resistant to hydrolysis [33]. By increasing the amount of lactic acid relative to glycolic acid one can easily increase the degradation timeframe from a few weeks to several months. Other techniques with blending polymers can be used to tailor the porosity, for instance by dissolving away one polymer to leave behind a more porous polymer structure [34-36]. Blending a degrading polymer with drugs or growth factors can cause the polymer to elute drugs or growth factors as the polymer degrades. This technique can be used to modify cell behavior around the scaffold, and to promote or hinder blood vessel formation.

Electrospinning is also unique among the micro- and nano-fabrication techniques described because one can alter fiber alignment during the spinning process. When nanofibers are electrospun, they are typically collected on an aluminum foil substrate as a random mesh, much like a pile of yarn. There are two techniques for aligning the nanofibers. One technique is to align the fibers as they form with electrostatic forces and a patterned dielectric gap collector. This technique is commonly referred to as plate electrospinning. Another technique is to align the fibers mechanically with a rotating mandrel, like winding yarn on a spindle as we 
have seen in Figure 1.5 [18]. Fibers can be collected on aluminum foil wrapped around the mandrel. Electrostatically aligned fibers are advantageous because the functional groups on the backbones of the polymers are aligned with respect to the electric field, a phenomenon which does not occur during rotating mandrel alignment [37-38]. Fibers collected on a rotating mandrel can be synthesized on a very large scale, on the order of hundreds of square centimeters versus those made on gap or dielectric collectors which are typically less than ten square centimeters in area. Sheets with collected nanofibers can then be unwrapped and used in cell culture. If the nanofiber collection is great enough scaffolds can be carefully removed or circularly wrapped from the sheet on the mandrel [9$10,39]$. Alignment of the nanofibers can be measured a number of ways. For the purposes of this research we will use the technique of measuring fiber angular deviation $(A D)$, which is the standard deviation of the angles of the nanofibers, so that a lower number defines a better aligned mesh. Examples of aligned and misaligned nanofibers are shown in Figure 1.5. 


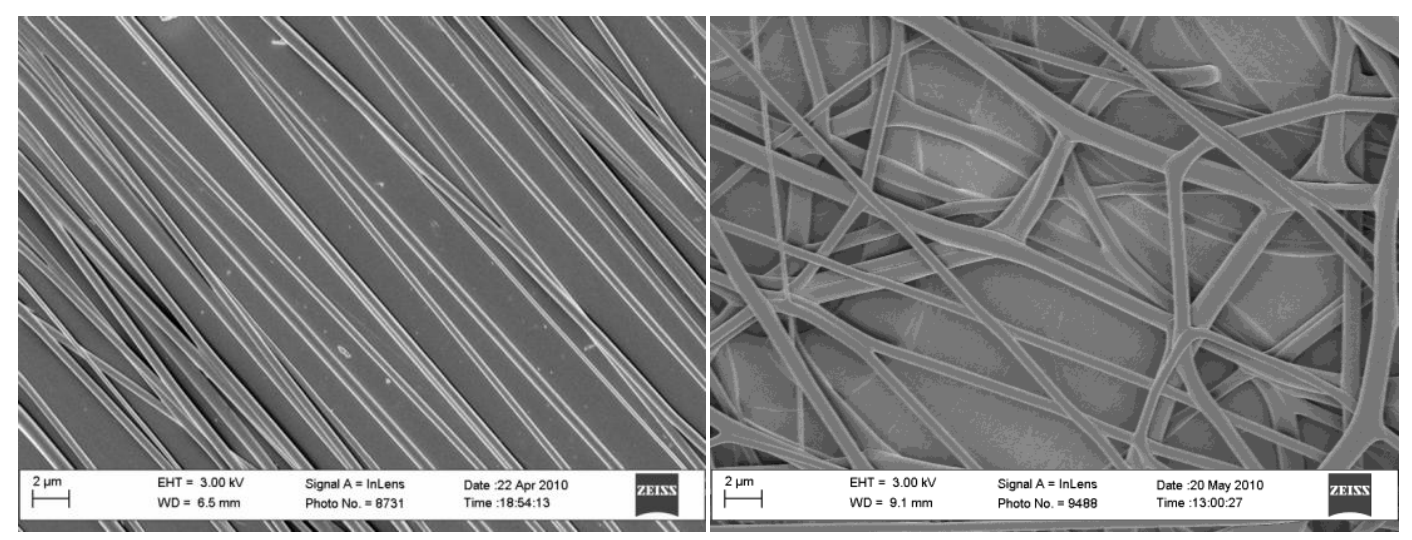

Figure 1.5: examples of aligned (right) and mis-aligned (left) nanofibers on a gold-coated Mylar ${ }^{\mathrm{TM}}$ substrate. The aligned fibers have an angular deviation of less than $5^{\circ}$.

\subsection{Creating smaller fibers}

Polymer nanofibers on the order of less than $200 \mathrm{~nm}$ have been successfully synthesized [27,40-41]. To reach these small scales, one or more of the following four techniques must be utilized: (1) decreasing the amount of polymer in solution, (2) increasing the charge in the solution, (3) decreasing the surface tension of the solution by the addition of a surfactant, or (4) increasing the dielectric properties of the solution. We shall discuss these in turn.

(1) The first is decreasing the amount of polymer in solution. This works in some cases, but when too low a concentration is reached, the polymer is no longer electrospinnable [18].

(2) Another option is increasing the charge of the polymer solution. This alteration can be done by the addition of a salt [42-43] or by increasing the charge density of the polymer, such as using a polymer with a net 
charge, such as polyphosphazene (PPHOS) with a charged nitrogen group, instead of a more typical neutrally charged polymer. Many salts have been studied, but the more notable ones are $\mathrm{Li}+\mathrm{Br}-, \mathrm{Na}+\mathrm{Cl}-$, and tetraethylammonium chloride (TEAC).

(3) Surfactants have also been studied to create smaller nanofibers. Surfactants can lower the surface tension of the polymer solution and thus lower its viscosity. Their surfactants allow the fiber to stretch more during flight (and thus become smaller in diameter) under the same electrostatic force.

(4) The final way of creating smaller fibers is to increase the overall dielectric constant of the polymer solution. This technique is most commonly performed by adding a small amount of a high dielectric solvent to a low dielectric solvent (i.e., by mixing two different solvents in the same solution). A solution with a higher dielectric coefficient stretches faster than a solvent with a low dielectric coefficient, because a greater internal electric field is formed in opposition to the applied electric field. Again, the greater stretching produces a narrower fiber. Changing the dielectric constant of the solution can be easily accomplished by adding di-methylformamide (DMF) to the electrospinning solution. DMF has a dielectric constant greater than 30 , and its addition will greatly increase a solution's typical dielectric constant of around 18. 


\subsection{Cellular interaction with nanofibers}

Cells in a growth medium will multiply through cell division. When a cell is introduced to a nanofiber substrate, its membrane expresses proteins so it can attach to the substrate. Recent work has shown that a cell will differentiate depending upon the substrate and nanostructured environment a cell encounters [44]. Fiber alignment is of great interest because cells tend to align themselves with respect to the direction of the fibers $[9,39,45]$. Aligned groups of cells are found in many ligament and nervous tissues. Research suggests if nanofibers are all aligned in a certain direction then the cells will orient, elongate, and align themselves with the nanofibers. Cells anchor themselves to the nanofiber meshes through anchoring proteins, such as vinculin. Vinculin is attached to actin and makes up the "endoskeleton" of the cell, providing it structure and support [46]. When a cell adheres to a nanofiber mesh, the cells will have different areas, different major axis/minor axis ratios (of an ellipse encompassing the cell), and different angular deviations. As the cells themselves multiply the new cells also align themselves with the nanofibers. The aligned fibers allow for a macroscopically aligned group of cells, and would be ideal for a ligament or

nervous tissue scaffold. Later chapters will explain how bi-polymer "coaxial" fibers can be synthesized and how these fibers may be used to deliver drugs. 


\subsection{Current state of the art in small, aligned nanofibers}

The best current researchers either have good control of alignment $[45,47-48]$ or nanofiber diameter [9,49]. Goldstein's group [9] was able to synthesize nanofibers of different diameters, but when their diameter decreased the angular deviation of the fibers increased to $30^{\circ}$. Other groups have synthesized small nanofibers $(60 \mathrm{~nm})$ with high alignment, but these techniques require the use of magnetic nanoparticles or other additives to the nanofibers, and these additives can be harmful to the cellular environment [50-51]. In addition, no one has synthesized small fibers of controllable diameter which also have good alignment. There is not currently reported in literature a detailed study on cellular response to the size effects of nanofibers of different sizes less than $1000 \mathrm{~nm}$ in diameter which maintains a high degree of fiber alignment $\left(<15^{\circ}\right)$ at this small size. 


\subsection{Conclusions}

In this chapter we introduced tissue engineering as a valuable approach to quicken the repair of damaged tissues. Electrospinning aligned nanofiber meshes to mimic the native ECM could be a valuable tissue engineering approach. There are several current methodologies to aligning nanofiber meshes, each with their respective advantages and disadvantages. Finally, the interaction of cells with their environments was discussed, as improved speed of cell growth and improved alignment of cell growth would likely lead to faster and better recovery. 


\section{Chapter 2: Aligning small and large nanofibers}

\subsection{Abstract}

In this chapter, we closely examine synthesis and alignment techniques of small (140nm) and large $(700 \mathrm{~nm})$ nanofibers. Current research indicates small, aligned nanofibers provide faster regeneration in tissue regeneration applications [52]. However, researchers have so far been unable to systematically synthesize nanofibers of selected smaller diameters $(<300 \mathrm{~nm})$ while maintain a high degree of alignment $\left(A D<15^{\circ}\right)$ without the use of nanoparticle additives to their electrospinning solutions [50-51]. In the following, we show how we solved these problems by creating a method by which one can tune nanofiber diameter to any desired size and overcome the challenges of aligning small nanofibers. We achieved this result through applications of our group's well-developed model of the stretching and repulsive electric fields present in electrospun nanofibers.

\subsection{Rationale for smaller nanofibers}

For the purposes of this research, nanofibers are considered fibers with a diameter of a few hundred nanometers. The natural extracellular matrix collagen and laminin - have some functional parts smaller than $100 \mathrm{~nm}$. In order to mimic the natural extracellular matrix $(E C M)$, nanofibers approximately 50 to $900 \mathrm{~nm}$ in diameter are needed [53]. Specifically, the small scale and surface area of these fibers are very similar to collagen I and laminin [9-10,53]. By 
replicating the surface characteristics of the ECM, researchers aim to quicken the tissue regeneration process.

Nanofibers on this scale can be produced readily by electrospinning polymers with specific solvents and conductivities, however, they are not well aligned $\left(A D \approx 20^{\circ}\right)[18,35]$. Solvents and conductivities must be carefully selected to create fibers on this scale. There is controversy in the current literature over whether nanotopographic cues from fibers less than $500 \mathrm{~nm}$ enhance $[39,45,52]$ or hinder cell adhesion $[9,35,54]$, with the majority of papers favoring fibers less than $500 \mathrm{~nm}$ stating it provides better nanotopography. The reason is that cues at the nanoscale enhance cellular adhesion [55]. One side of the debate suggests that the cells will anchor easily to the smaller fibers and align themselves accordingly. The other side states that as the fibers get smaller, in other words, the cells have fewer attachable focal adhesion sites and will therefore have more difficulties in aligning $[9,40]$.

\subsection{Creating small aligned nanofibers}

Current researchers are creating smaller aligned nanofibers with a variety of electrospinning alignment techniques: patterned plate collectors, a rotating mandrel, dielectric substrates, and nanoparticles in the nanofibers. Research groups are approaching the sub $60 \mathrm{~nm}$ size and good alignment with a variety of polymer types. There are, however, many difficulties associated with creating smaller, aligned nanofibers as evident in Figure 2.1. 


\section{Diameter vs AD for all aligned nanofibers}

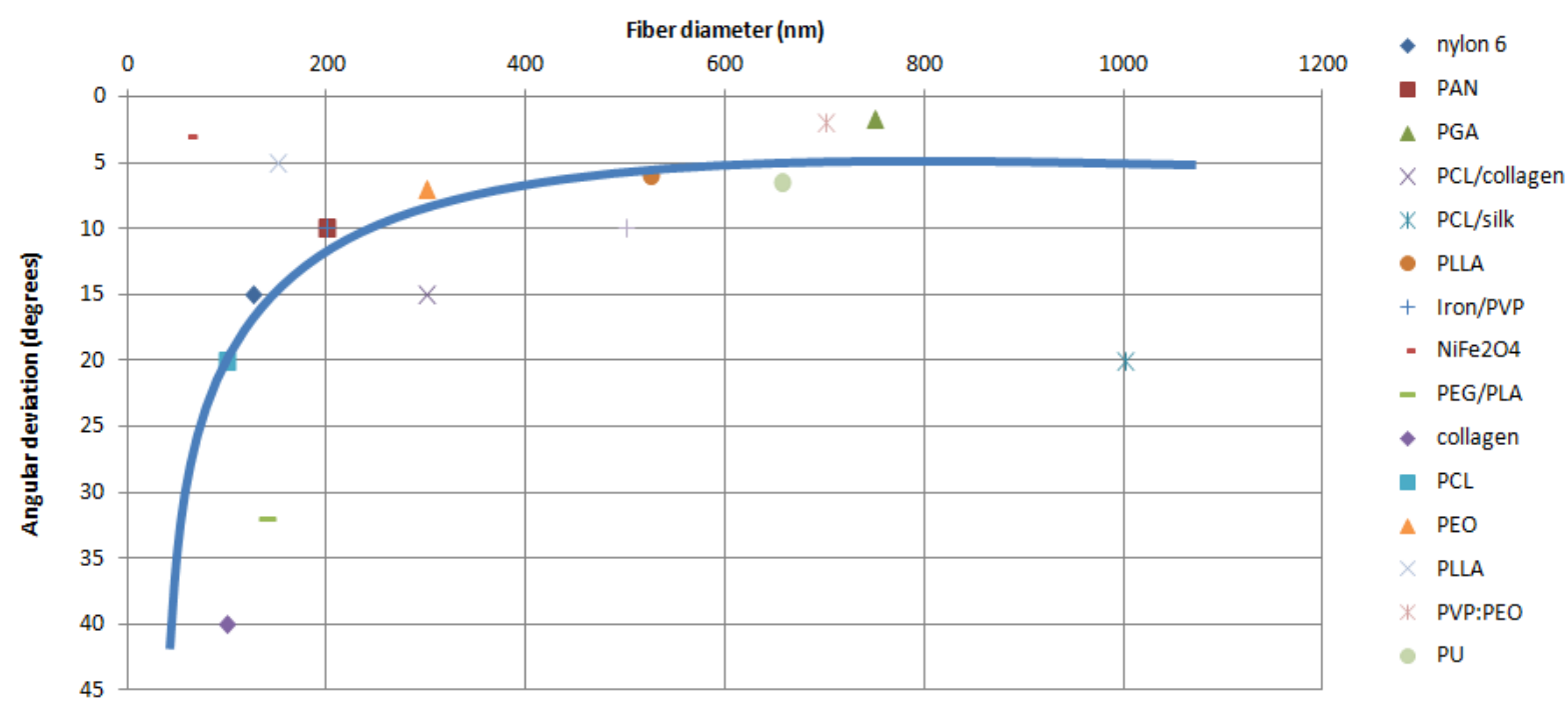

Figure 2.1: Lack of highly aligned $\left(A D<15^{\circ}\right)$, small $(300 \mathrm{~nm})$ nanofibers in the literature. Graph shows fiber diameter vs. angular deviation for a variety of polymer types. Standard deviation bars were omitted for cleanliness. Exceptionally well aligned, small fibers are created with magnetic nanoparticles. An approximate polynomial based curve has been fitted to the data points. Note how angular deviation increases for small (sub-300nm) nanofibers. Figure based on survey of literature [26-27,29-31,39,45,48-51,56-61].

One can see from Figure 2.1, that as nanofibers get smaller, they have a tendency to be misaligned. The exceptions in Figure 2.1 in the sub $200 \mathrm{~nm}$ yet highly aligned fiber regime utilize plate electrospinning with magnetic iron nanoparticles [27] and thermosetting by heating well aligned 1.3 micron fibers into smaller aligned nanofibers [26]. Other groups have used plate collectors to synthesize aligned nanofibers $\sim 60 \mathrm{~nm}$ in diameter with less than 8 degrees 
angular deviation; however, these techniques require the use of magnetic or other additives to the nanofibers, and these additives can be harmful to a cellular environment [50-51].

Small, highly aligned nanofibers have not been synthesized using mandrel alignment techniques. When small nanofibers are synthesized using this technique, they are typically quite misaligned [9]. The reasons for the difficulties in aligning smaller nanofibers are not well understood or explained in the literature. Current research has also shown that by using dielectric collectors with dielectric constants around $10\left(\varepsilon_{r} \approx 10\right)$ yield highly aligned nanofibers, but aligning smaller fibers with dielectric materials has not been thoroughly explored [62].

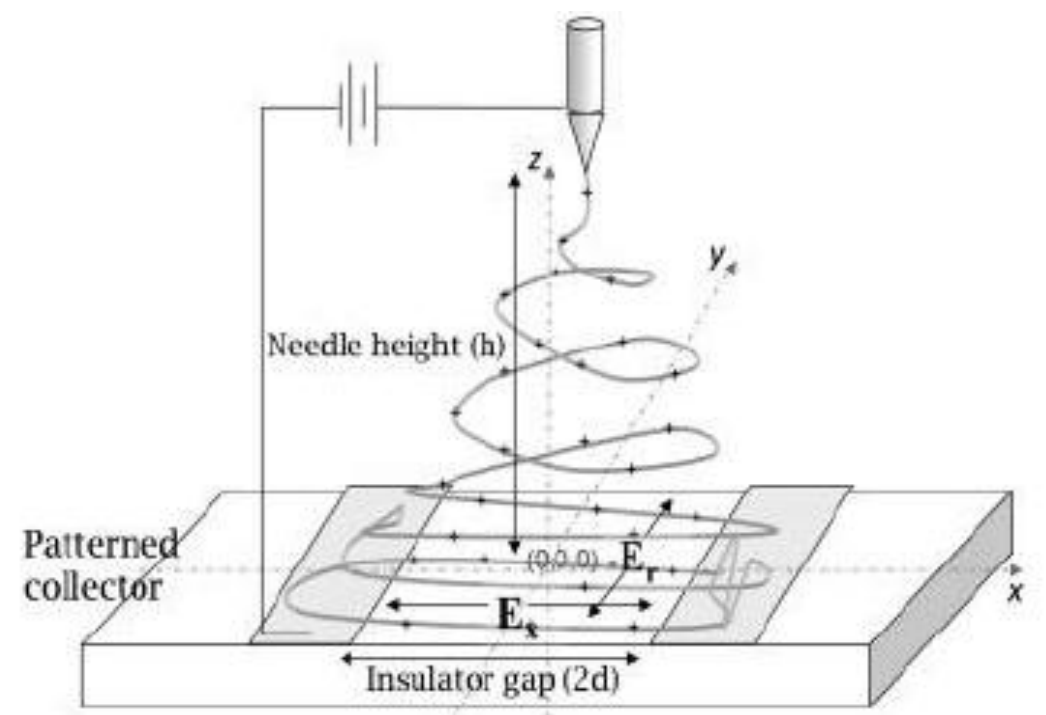

Figure 2.2: Electrospinning on a patterned collector. The electrospinning needle is located at the top, with the grounded patterned collector at the bottom of this image. Fibers are extruded from the needle, experience a high electric field in the form of a high voltage applied from the needle to the grounded collector. Note the stretching force on the fibers, $E_{x}$, and the repulsive force between the fibers, $E_{r}$ [63]. 
As described in Chapter 1, electrospinning has a large area of instability in which the polymer and solvent loop back and forth as nanofibers are formed. This instability region needs to be overcome with one of the electrospinning alignment techniques such as electrostatic plate gap, mandrel, patterned mandrel, or dielectric block. Since most polymers used are non-conducting, they retain their electrical charges and thus they form charged fibers on the collector. This charged layer interferes with the alignment of additional incoming nanofibers, because the incoming nanofibers have the same positive charge and they are repelled by the fibers already present [63].

In Figure 2.2, it is seen that there are two forces in the electrospinning process, $E_{x}$ and $E_{r} . E_{x}$ is the electric field which causes a stretching force on the electrostatically charged nanofiber towards the metallic edges of the grounded collector. This force is present only very near the edges of gap or dielectric collectors. $E_{r}$ is the repulsive force between undischarged deposited nanofibers, and is present in all electrospun nanofibers especially where the stretching electric field is at a minimum, in the center of the gap collector. Without careful adjustment of $E_{x}$ and $E_{r}$, nanofibers will collect unaligned. Intuitively, a smaller gap width will result in a stronger $E_{x}$ (large gap widths do not align nanofibers). The stretching electric field is depicted in Figure 2.3 


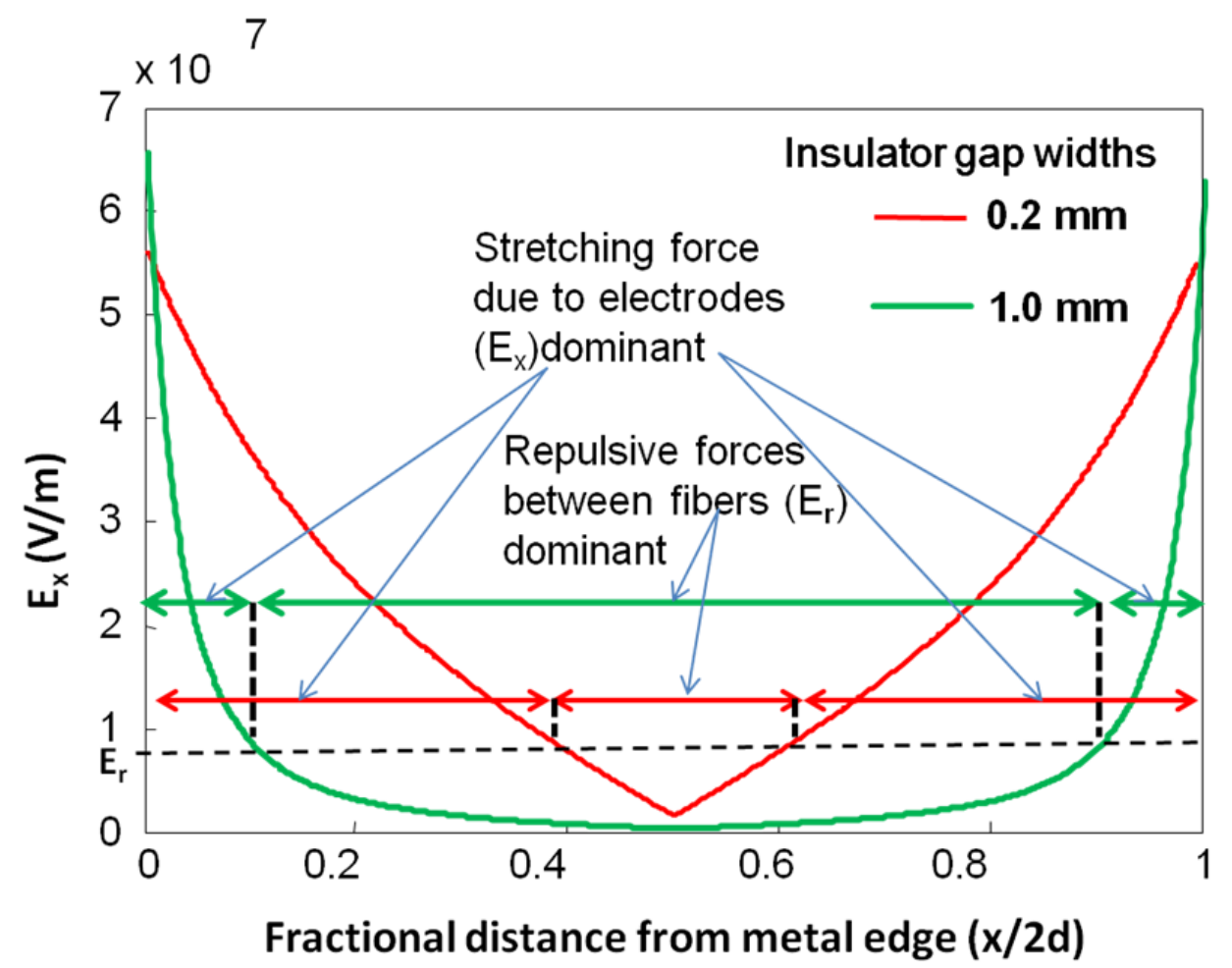

Figure 2.3: Graphical representation of $E_{x}$ and $E_{r}$. The stretching forces are dominant over a greater spatial extent for smaller gap widths [63].

From Figure 2.3, one can see that the stretching force is dependent on the insulator gap width. Larger gap widths provide less of a stretching electric field (lower $E_{x}$ ), while smaller gap widths provide a greater electric field (higher $E_{x}$ ). At the center of the gap width, the electric field is at a minimum (seen by the apex of the parabolas). Therefore, fibers at the center of the gap only experience the repulsive forces from deposited nanofibers. A nanofiber approaching the center of the gap would be repelled by the electric field due to the residual charge, $E_{r}$, and then pulled towards either edge by the stretching electric field, $E_{x}$. This figure also shows increasing $E_{x}$ through decreasing gap width is more effective than 
decreasing $E_{r}$ because of the larger spatial width over which the stretching field exists.

\subsection{Polymer selection}

A key factor in determining the biocompatibility of a nanofiber mesh is the inherent properties of the selected polymer. This is because the biocompatibility of the mesh structure is governed entirely by the interactions of cell anchoring proteins with the nanofiber mesh. Not all common polymers are biocompatible. The polymer used in creating nanofibers is the most important aspect of a nanostructure [64]. A biocompatible material elicits little to no immune response after implantation [17]. Common polymers, such as poly-vinyl-pyrrolidone (PVP), may be readily electrospun into aligned structures [51], but this polymer is not especially biodegradable and may require the addition of other compounds to its spinning solution to improve its biocompatibility and degradation properties [65$66]$.

Some polymers are stable forever in the tissue. Other polymers are biodegradable, that is, they are broken down in the tissues by hydrolysis or enzymes. These polymers can be tailored to degrade as the tissue self-repairs. One must also consider the potential toxicity of the degradation byproducts and their effects on the surrounding tissue, because it is important that the byproducts are non-toxic and non-inflammatory. For this work, we chose polylactic-co-glycolic acid (PLGA) because of its biocompatibility and the limited toxicity of its degradation byproducts: lactic and glycolic acids [33]. These acids 
occur naturally in the human body, and they are readily broken down in enzymatic reactions in the human body, and although they may slightly lower the local $\mathrm{pH}$, slightly increased concentrations of these weak organic acids are benign. PLGA is unique in the sense that one can tailor its degradation rate simply by altering the ratio of lactic to glycolic acid, as discussed Chapter 1 Part 7: nanofiber synthesis. Although lactic acid has a very similar chemical structure to glycolic acid, the additional methyl $\left(\mathrm{CH}_{3}\right)$ group makes lactic acid less susceptible to hydrolysis, the primary mechanism of degradation for PLGA in aqueous environments (i.e., the body).

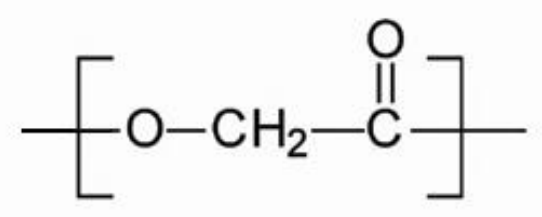

PGA

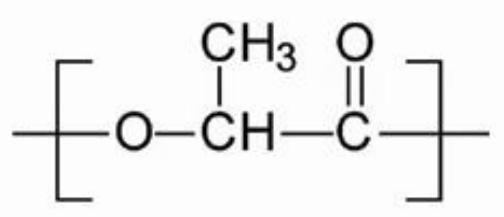

PLA

Figure 2.4: poly-glycolic acid (PGA) and poly-lactic acid (PLA). Note the extra methyl group $\left(\mathrm{CH}_{3}\right)$ present in PLA.

By increasing the amount of lactic acid in the polymer one can slow down the polymer's degradation in vivo [33]. PLGA can be denoted with a simple ratio that represents the percent lactic and glycolic acids. The formula is commonly written as:

Eq. 2.1

PLGA L:G 
where $L$ is the percent lactic acid and $G$ is the percent glycolic acid. For example PLGA 50:50, with 50\% lactic acid and 50\% glycolic acid, has a degradation timeframe of a few weeks, as opposed to PLGA 85:15 which degrades over several months [33,67-68]. PLGA nanofibers have been examined for their use in a variety of implants. Previous research has aligned PLGA using plate, mandrel, patterned mandrel, and dielectric block techniques [9,41,47,69-72].

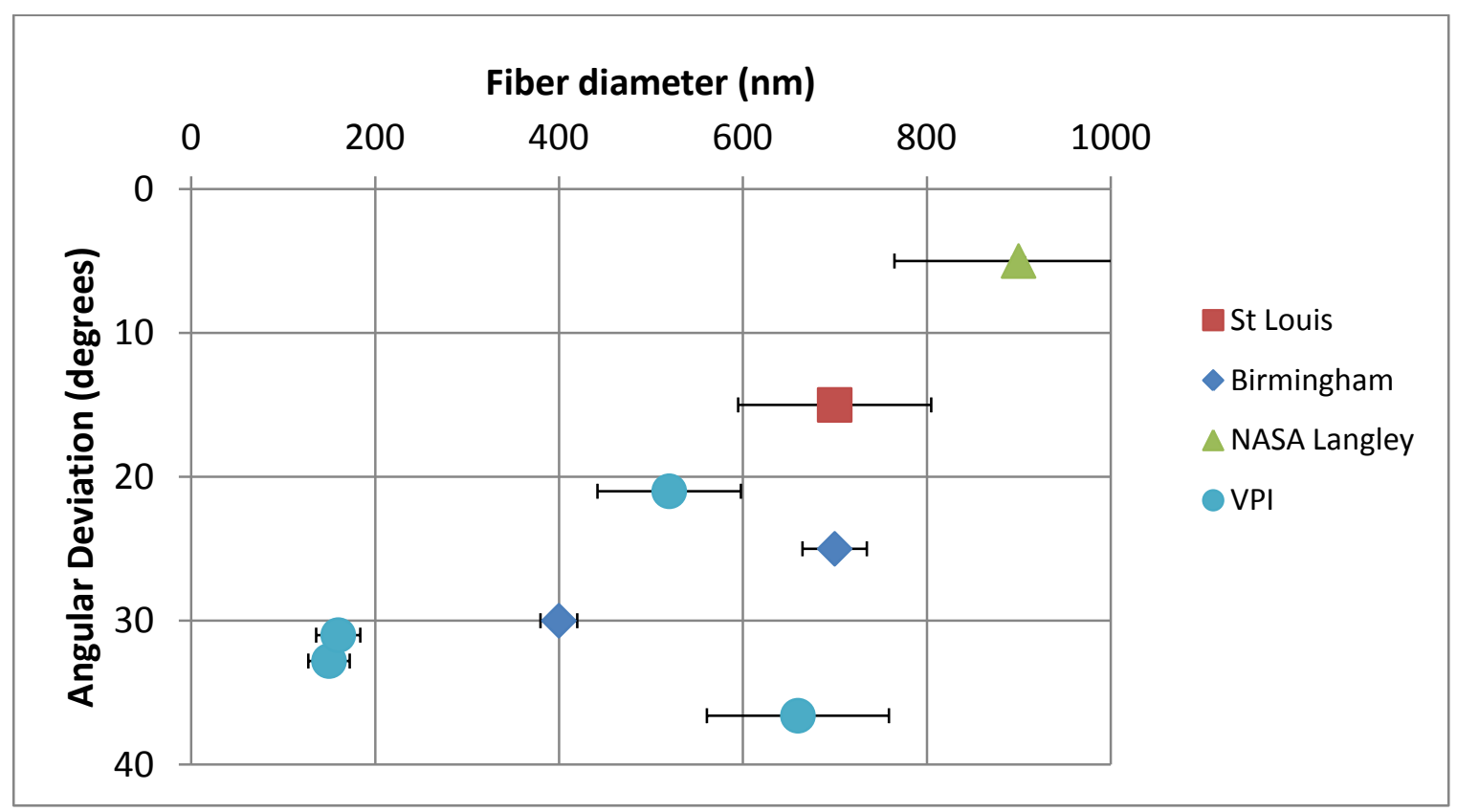

Figure 2.5: fiber diameter vs angular deviation for PLGA nanofibers aligned with both plate and mandrel techniques as reported in literature. Figure created from survey of literature $[9,41,56,70]$.

As one can see from Figure 2.5, PLGA nanofibers have been synthesized down to sub-200nm, but the angular deviation is around $30^{\circ}$. Currently, no small (less than $300 \mathrm{~nm}$ ), highly aligned $\left(\mathrm{AD}<15^{\circ}\right)$ PLGA nanofibers have been described in the literature. 
There is also no systematic way to vary fiber diameter described in literature. Most groups have polymer recipes which vary polymer concentration to yield different nanofiber diameters $[9,18,47,73]$, however, varying the polymer concentration does not always correlate to predictably smaller fiber diameters. It has been hypothesized that varying the dielectric constant of polymer solutions would yield different sized nanofibers [18,73-74], however it has yet to be systematically explored. Ideally, one would have a formula by which any desired diameter nanofiber could be readily synthesized.

In our experiments, we created highly aligned PLGA 85:15 nanofibers with different diameters and angular deviations through altering the dielectric constant of the polymer solution. We chose PLGA 85:15 because its longer degradation times make it more suitable for implantation. In this research, we created aligned nanofibers through the use of four different alignment techniques: electrostatic, mandrel, patterned mandrel, and dielectric block.

\subsection{Materials and methods for creating successively smaller nanofibers}

PLGA 85:15 was dissolved in varying mixtures of tetrahydrofuran (THF) and dimethylformamide (DMF). The ratio of THF:DMF was altered to change the dielectric constant of the polymer solution. Altering the ratio of THF:DMF acts to increase or decrease the effective dielectric constant of the electrospinning solution and therefore allows $E_{x}$ to have a greater or lesser effect. Tetraethylammonium chloride (TEAC) was added to the solution in a few 
milligram per $\mathrm{mL}$ quantities to further increase the solution's charge and decrease viscosity. It has been shown previously that adding TEAC helps not just to reduce beading, but also to decrease fiber diameter because TEAC acts as a surfactant to enable easier stretching of the nanofiber in flight [teac sources]. HFIP was also used as a solvent to synthesize nanofibers. Previous work $[47,56,75]$ has shown that HFIP gives very well aligned fibers. DMF and TEAC were added to polymer solutions with HFIP as the primary solvent to lower fiber diameter. After creating our model for varying diameter of PLGA nanofibers, we will compare PLGA nanofiber diameters to similarly electrospun GEG PhPh nanofiber diameters to observe if this method is applicable to different polymers.

After reviewing the literature, we decided to use Mylar ${ }^{\mathrm{TM}}$ as our collecting substrate because it would best provide mechanical support for our nanofiber meshes and not interfere with cellular adhesion. Mylar ${ }^{\mathrm{TM}}$ makes an ideal substrate because it is transparent, and thus good photomicrographic images can be taken during later cell culture experiments. The substrates used for fiber collection were either Mylar ${ }^{\mathrm{TM}}$, gold/titanium coated Mylar ${ }^{\mathrm{TM}}$, or aluminum foil. Gold/titanium coated Mylar ${ }^{\mathrm{TM}}$ is semi-transparent, as the coating is only $50 \mathrm{~nm}$ thick. Its conductivity allows the incoming nanofibers to discharge, and the loss of charge further assists nanofiber alignment. Dielectrically aligned nanofiber samples required no substrate as they were collected in air between the two dielectric blocks.

After their creation, the nanofibers were then dried under a $10^{-4}$ Torr vacuum at $22^{\circ} \mathrm{C}$ for at least 24 hours. Finally, samples approximately $1 \mathrm{~cm}^{2}$ were taken from 
these nanofibers for Scanning Electron Microscope (SEM) analysis and the cell studies presented in Chapter 4. The typical mesh thickness of the nanofiber samples is very small, on the order of a millimeter or a few thousand layers of nanofibers. Samples for SEM were sputter coated with a $12 \mathrm{~nm}$ thick film of gold/palladium to make the nanofibers electrically conductive, a requirement for good SEM images. The resulting images were analyzed using ImageJ [61] and Microsoft Excel for fiber diameter and angular deviation.

\subsection{Aligning nanofibers}

Nanofibers were aligned using one of four different alignment techniques: insulator gap plate collectors, rotating mandrel, rotating patterned mandrel, and dielectric block.

\subsubsection{Insulator gap plate collectors}

Insulator gap plate collectors were used in aligning many of the fibers in Figures 2.1 and 2.5. A plate collector is two conductive strips separated by an insulating gap. Additional research has shown that nanofibers will also align with an air gap and a plate collector made from material with a dielectric other than air [62]. Our research has led us to create plate collectors by patterning gold and titanium on Mylar ${ }^{\mathrm{TM}}$. 


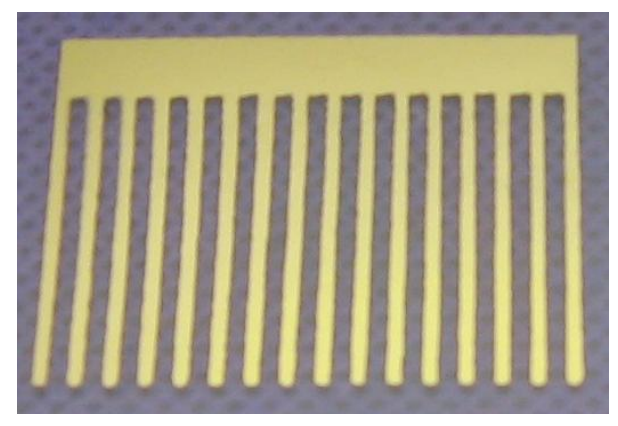

Figure 2.6: A patterned Mylar ${ }^{\mathrm{TM}}$ plate collector, made from gold/titanium used for plate electrospinning. The clear plastic film is not visible in this image. The gap width is $2 \mathrm{~mm}$.

To make a patterned Mylar ${ }^{\mathrm{TM}}$ collector, one must first use a laser cutter to pattern the fingers in another sheet of Mylar ${ }^{\mathrm{TM}}$ to use as a mask in evaporation. We used a $\mathrm{CO}_{2} 50$ watt laser, model number $x 660 \mathrm{M}$ to cut fingers in the layer of Mylar ${ }^{\mathrm{TM}}$. The Mylar ${ }^{\mathrm{TM}}$ was adhered to a moistened cardboard backing to help dissipate heat from the laser and cool the Mylar ${ }^{\mathrm{TM}}$. This laser patterned "mask" of Mylar ${ }^{\mathrm{TM}}$ is taped onto un-patterned clear acetone washed Mylar ${ }^{\mathrm{TM}}$. 10nm of titanium followed by $50 \mathrm{~nm}$ of gold are evaporated on both the patterned and un-patterned Mylar $^{\mathrm{TM}}$. This procedure is done under high vacuum with electron beam evaporation techniques. Finally, the laser patterned Mylar ${ }^{\mathrm{TM}}$ is removed, yielding the patterned Mylar ${ }^{\mathrm{TM}}$ seen in Figure 2.6. Each patterned Mylar ${ }^{\mathrm{TM}}$ sheet has several patterned collectors. The gap width is constant on each sheet, and it ranges from $2 \mathrm{~mm}$ to $5 \mathrm{~mm}$. This technique can also be used to create unpatterned gold-titanium Mylar ${ }^{\mathrm{TM}}$ for use with rotating mandrels. By lowering the gap width from $5 \mathrm{~mm}$ to $2 \mathrm{~mm}, \mathrm{E}_{\mathrm{x}}$ is increased. This approach is depicted in Figure 2.3. The aim is to increase alignment by increasing the stretching electric field on the incoming nanofibers. 
Fibers grown though plate and electrospinning have oriented polymer groups [37-38]. This orientation occurs because of the repulsion of like-charged groups on the polymer backbone [37-38,76-80]. Not only does the incoming fiber layer not only aligns itself to the previously deposited fiber layer, but charges within the backbone of the polymer, for instance, methyl or $\mathrm{C}=\mathrm{O}$ groups, orient themselves with respect to the applied electric field, to the most energetically favorable state. This gives molecular oriented "hot spots" throughout the fiber mesh, which enhance cellular adhesion [55]. This issue is further discussed in Chapter 4.

\subsubsection{Mandrel collection}

For this research, we used a repurposed Sherline 4400 lathe with a high speed adapter to serve as our rotating mandrel. This lathe is capable of rotating a $5 \mathrm{~cm}$ in diameter cylindrical rod up to $10000 \mathrm{rpm}$. The nanofibers were collected on this grounded mandrel at $6000 \mathrm{rpm}$. We used a variety of solid and hollow core mandrels. One type of mandrel used was a $5 \mathrm{~cm}$ in diameter hollow plastic tube. This mandrel was then wrapped with grounded aluminum foil. Clear, uncoated Mylar ${ }^{\mathrm{TM}}$ was then wrapped on the grounded aluminum foil to allow for easy transfer of the nanofibers to SEM and cell culture. In other experiments, we used a solid gold/titanium coated Mylar ${ }^{\mathrm{TM}}$ sheet to collect nanofibers without an insulating Mylar ${ }^{\mathrm{TM}}$ layer between the nanofibers and our ground. In mandrel electrospinning, there is no stretching force due to a patterned electric field. The fibers are only aligned from the shear force from the mandrel and by 
manipulating the residual charge. We varied residual charge on the deposited nanofibers through the ionic salt additive, TEAC, to determine how to synthesize optimally aligned, small nanofibers.

\subsubsection{Patterned mandrel collection}

Additionally, patterned conductive strips can be attached to the Mylar ${ }^{\mathrm{TM}}$ film to combine both the rotational alignment forces from mandrel collection with the electrostatic alignment forces. Sheets of Mylar $^{\mathrm{TM}}$ as seen in Figure 2.7 are wrapped around an insulating mandrel. Care is taken to ground each $1 \mathrm{~cm}$ wide conductive strip to ensure proper fiber collection. The chuck on the front end of the mandrel is grounded, and a resistance of less than 8 ohms from the metal strips to common ground was verified before each run. We tested patterned mandrels because in theory, they should align nanofibers better than solid mandrels. The reason is that fiber collection in this case relies on the combination of both electrostatic attraction of the nanofiber to the four different ground points and the shear force of the rotating mandrel [81]. 


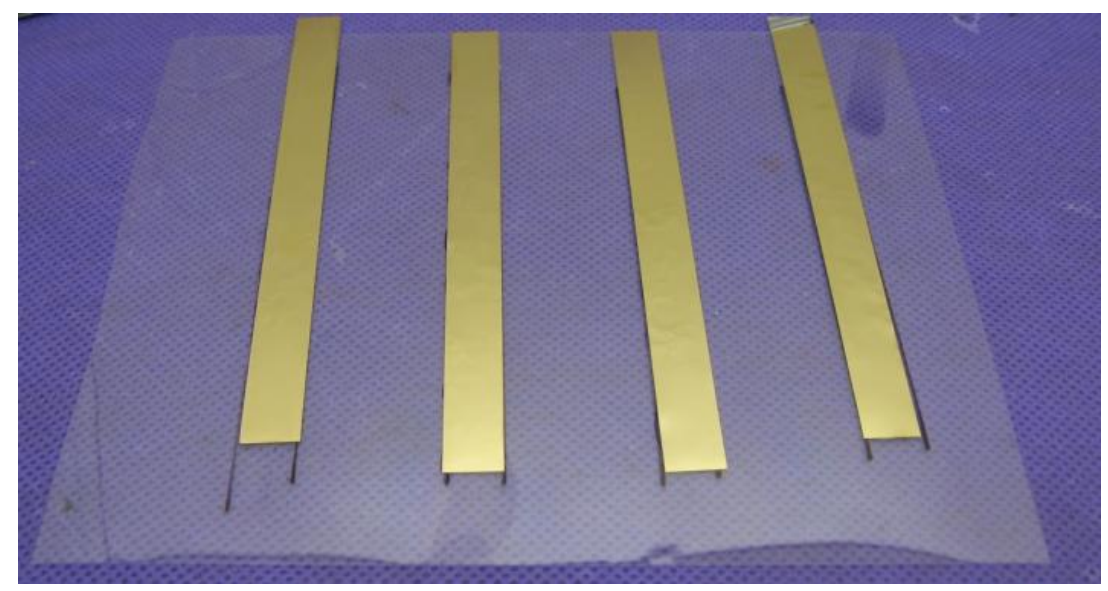

Figure 2.7: patterned Mylar. Gold/titanium coated Mylar is glued onto a sheet of Mylar. The conductive strips are $1 \mathrm{~cm}$ in width and are spaced with a $2.5 \mathrm{~cm}$ gap between strips. The sheet is wrapped widthwise around the mandrel such that the strips are parallel to the axis of rotation and conductive strips are grounded.

\subsubsection{Dielectric block collection}

For this research, we used materials exhibiting different dielectric constants. The collectors used were $2.5 \mathrm{~cm} \times 2.5 \mathrm{~cm} \times 10 \mathrm{~cm}$ rods of (1) wood $\left(\varepsilon_{r} \approx 2-8\right)$, (2) aluminum ( $\varepsilon_{1} \approx 8-10$, ignoring complex components), or (3) AINiCo magnets $\left(\varepsilon_{\tilde{r}} \approx 12.5-13\right)$. (Note that the dielectric constant of a material, $\varepsilon_{\mathrm{r}}$, is not the same as the electric field from charged, deposited nanofibers, $E_{r}$.) The materials were spaced between .7 and $3 \mathrm{~cm}$ apart to create a patterned dielectric air gap. The ceramic magnets were spaced with non-magnetic sticks and secured with strong tape, as the magnetic field was on the order of 2 Tesla [47]. By changing the dielectric constant of the material, $E_{x}$ can be increased (materials with higher dielectric strength) or decreased (materials with lower dielectric strength). $E_{x}$ can be further increased by decreasing the air gap between the dielectric materials. It was hypothesized in [47] that the magnetic field aids in alignment of nanofibers. 
Other collectors such as wood and aluminum have magnetic permeability but are considered non-magnetic. We examined nanofiber collection on each of these collectors to determine both if magnetic electrospinning is a valid hypothesis, and attempt to determine which dielectric provides optimal fiber alignment. We also attempted to align smaller nanofibers using dielectric block collectors.

\subsection{Varying the residual and stretching electric fields}

We varied $E_{r}$ by (1) increasing the charge in the polymer solution by adding the salt TEAC, and (2) utilizing a solvent with a higher vapor pressure.

To explore the effect of TEAC on the alignment of nanofibers on dielectric blocks, 3:1 THF:DMF nanofibers with concentrations of TEAC of 1 or $2 \mathrm{mg} / \mathrm{mL}$ were electrospun on dielectric wooden block collectors with varying gap width between $0.5 \mathrm{~cm}$ and $2 \mathrm{~cm}$. The effect of decreasing gap width increases the effect $E_{x}$ has on the incoming nanofiber by allowing greater stretching across the entire collector, as seen in Figure 2.3.

The vapor pressure of the polymer solvent was changed to allow the solvent to leave the nanofibers more quickly, thereby trapping residual charge in the deposited nanofiber layer. If there is solvent left in the nanofibers after electrospinning, it is possible that the solvent will allow the nanofibers to discharge to the ground, as the solvent is a conductive fluid and it will create a 
conduction pathway, thus the nanofibers would have a smaller residual charge[mol orient pbt NFs]. For this set of experiments, $20 \%$ weight/volume of PLGA was dissolved in $\operatorname{HFIP}\left(\varepsilon_{r}=16.75\right.$, vapor pressure $\left.=120 \mathrm{mmHg}\right), 2: 1$ THF:DMF ( $\varepsilon_{\mathrm{r}} \approx 17$, vapor pressure $\left.\approx 96 \mathrm{mmHg}\right)$, and 3:1 THF:DMF $\left(\varepsilon_{\mathrm{r}} \approx 15\right.$, vapor pressure $\approx 107 \mathrm{mmHg}$ ) to compare solvents with similar dielectric strength but different vapor pressures. Ambient temperature in our basement laboratory was constant at $69.5^{\circ} \mathrm{F}$. The working distance used was $10 \mathrm{~cm}$, voltage of $20 \mathrm{kV}, 21$ gauge needle, and flow rate of $0.3 \mathrm{~mL} / \mathrm{hr}$.

\subsection{Results}

First, a method to reliably reproduce nanofibers of different diameters was discovered by varying the ratio of THF:DMF in the polymer solvent with or without the presence of TEAC. Initially it was found that nanofibers of different diameters were produced in a straightforward fashion when the ratio of THF:DMF was varied, with or without TEAC. These results are listed in Table 2.1.

\begin{tabular}{|c|c|c|}
\hline THF:DMF ratio & TEAC $\mathbf{( m g} / \mathbf{m L})$ & Fiber diameter \pm S.D.(nm) \\
\hline $1: 1$ & 2 & $150 \pm 46$ \\
\hline $1: 1$ & 1 & $140 \pm 35$ \\
\hline $2: 1$ & 2 & $260 \pm 50$ \\
\hline $2: 1$ & 1 & $340 \pm 240$ \\
\hline $2: 1$ & 1 & $270 \pm 98$ \\
\hline $3: 1$ & 1 & $450 \pm 370$ \\
\hline $3: 1$ & 0 & $700 \pm 310$ \\
\hline
\end{tabular}

Table 2.1: Electrospun fiber diameter and angular deviation aligned with the mandrel. 
Nanofibers were electrospun with each of the four different alignment techniques to determine which technique provided the best results given the fiber diameter.

Figure 2.8 contains nanofibers electrospun on gold coated Mylar ${ }^{\mathrm{TM}}$ plate collectors with diameters which varied depending on the solvent ratio in the polymer solution. Note how smaller nanofibers typically have larger angular deviations except in certain cases.

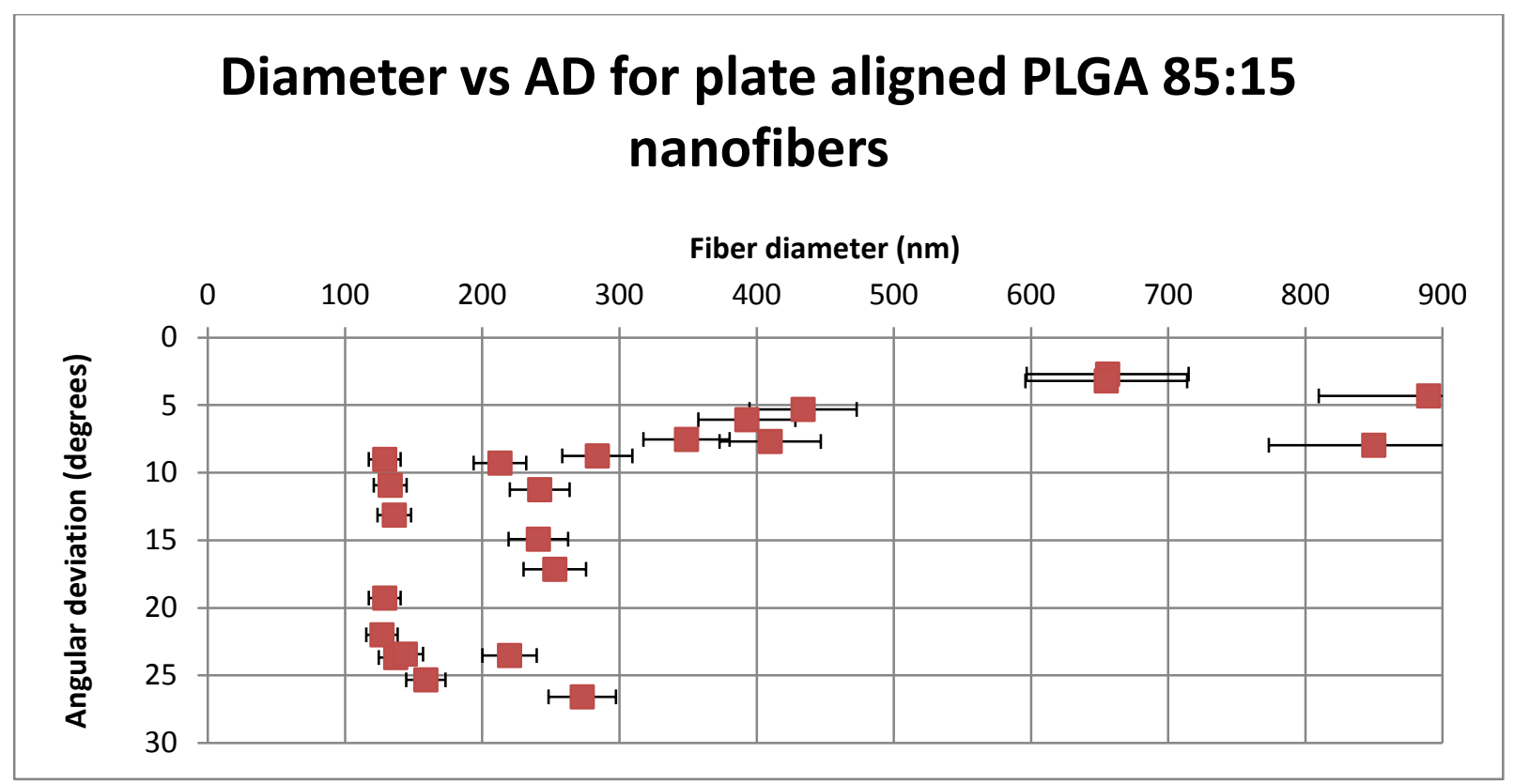

Figure 2.8: Nanofibers grown on gap collectors. Note fibers retain good alignment $\left(<10^{\circ} A D\right)$ even when fiber diameter dropped below 200nm. Each sample point is from a different electrospinning run carried out in recent experiments.

Nanofibers ranging from $140 \mathrm{~nm}$ to $700 \mathrm{~nm}$ were successfully created and aligned by the mandrel by varying the ratio of THF:DMF and adding TEAC. The diameter of the nanofibers decreased as more DMF was added to the solution. The 
smallest nanofibers were highly sensitive to gap width, collecting well only on the smallest collectors (2mm gap size). The medium-sized nanofibers collected well on the mid range ( $3 \mathrm{~mm}$ gap size). This is in agreement with our model: smaller nanofibers require a small gap width.

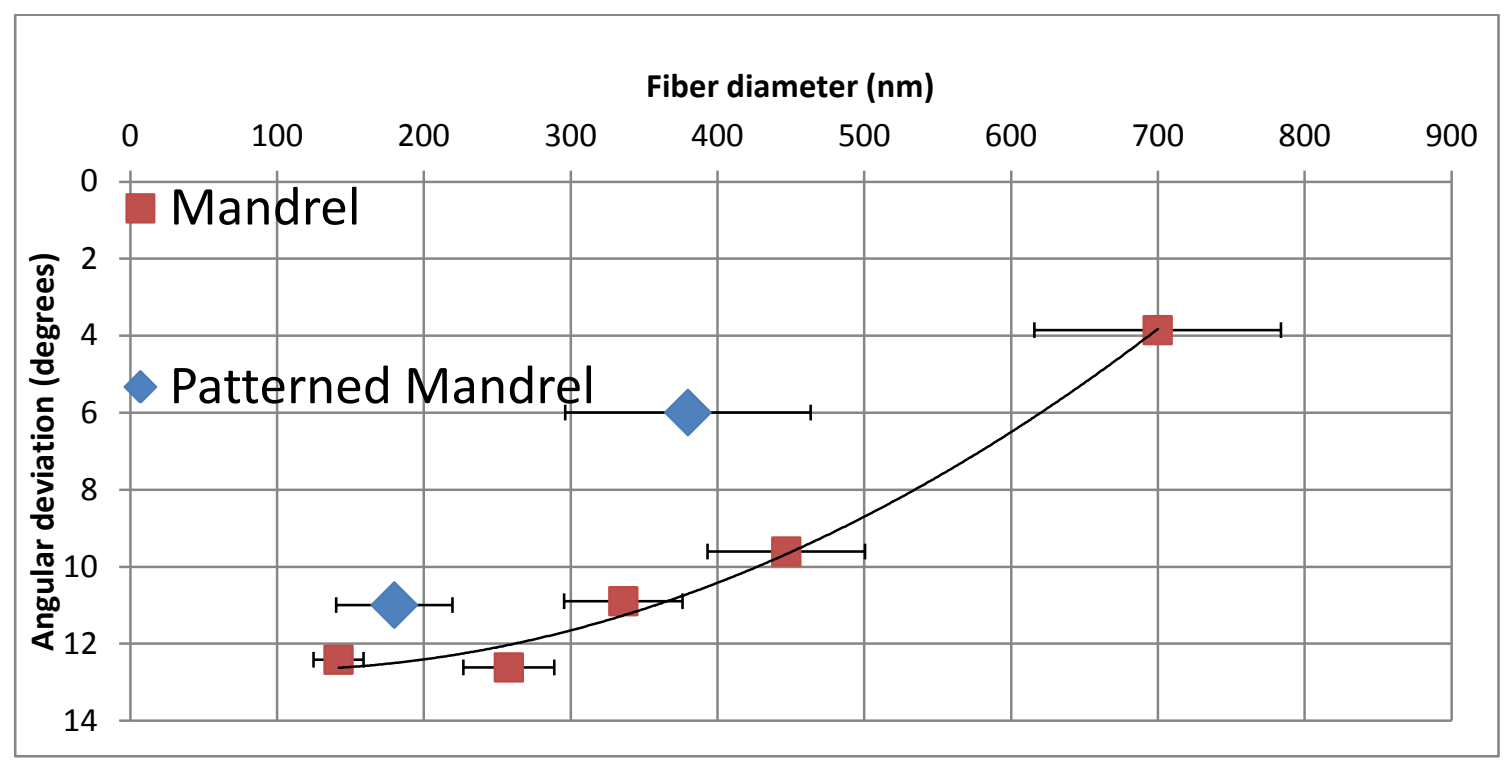

Figure 2.9: Graph of the electrospun nanofibers' diameter versus the nanofibers' angular deviation for nanofibers collected on a smooth, non-conducting surface on a conducting, rotating mandrel and patterned mandrel. Note the poorer alignment (increase in Angular Deviation beyond 10 degrees) when the nanofiber diameter falls below $400 \mathrm{~nm}$ and improved alignment for smaller size fibers on the patterned mandrel. 


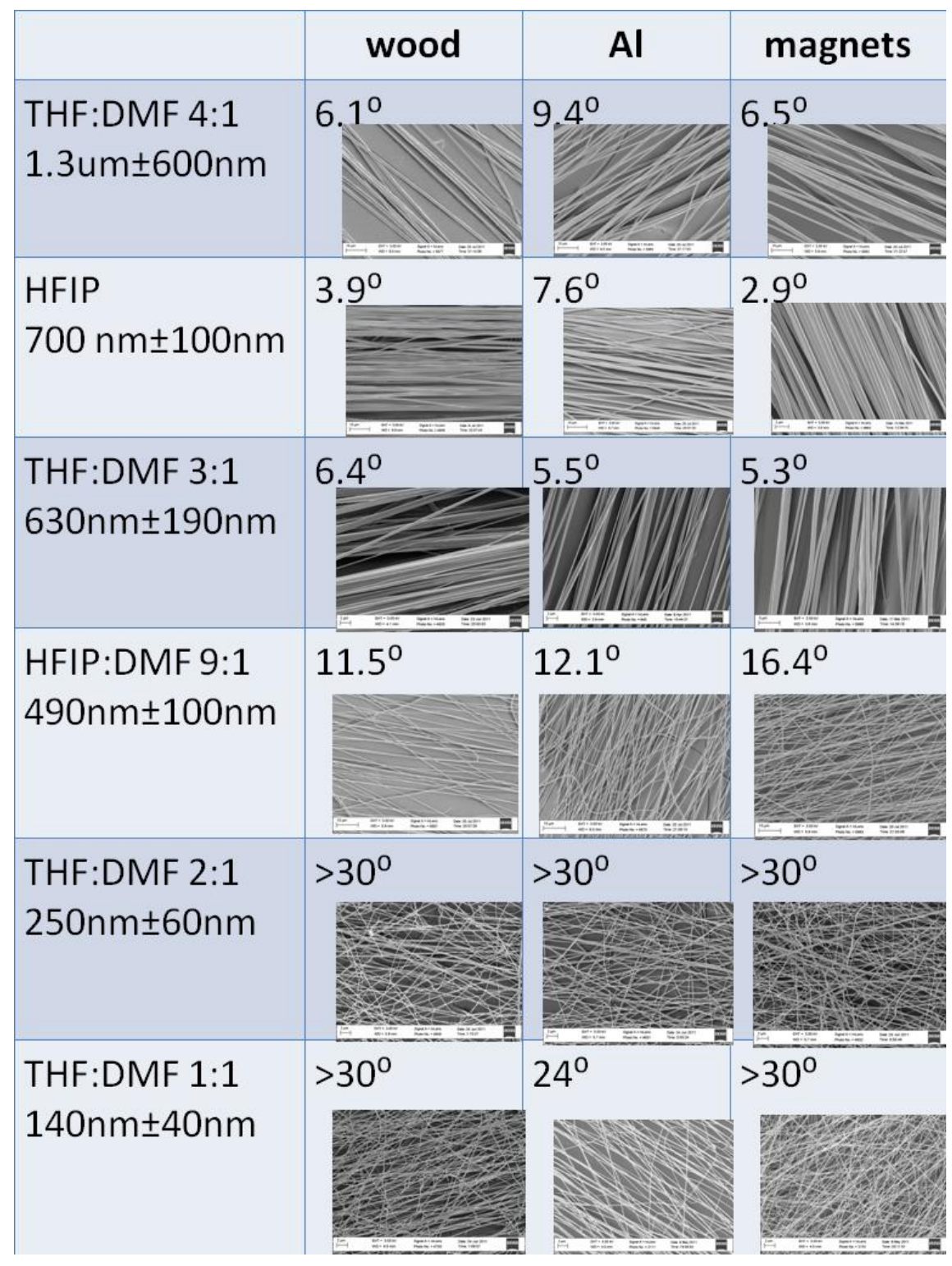

Figure 2.10: Nanofibers of varying size grown on different dielectric materials of increasing dielectric strength spaced at $1 \mathrm{~cm}$. 


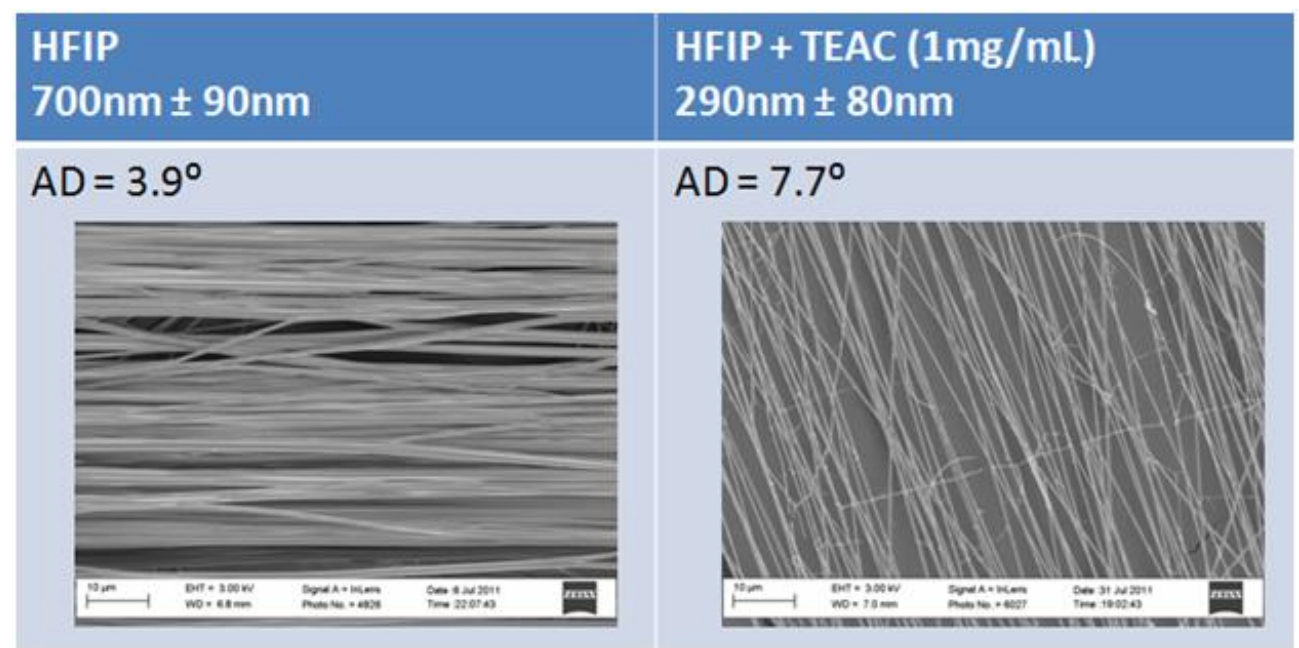

Figure 2.11: Adding TEAC to decrease fiber diameter and increase residual charge. These nanofibers were grown on dielectric wooden blocks at a width of $1 \mathrm{~cm}$. Note the decrease in diameter by the addition of TEAC alone. Also note the increase in Angular Deviation with the drop in fiber diameter.

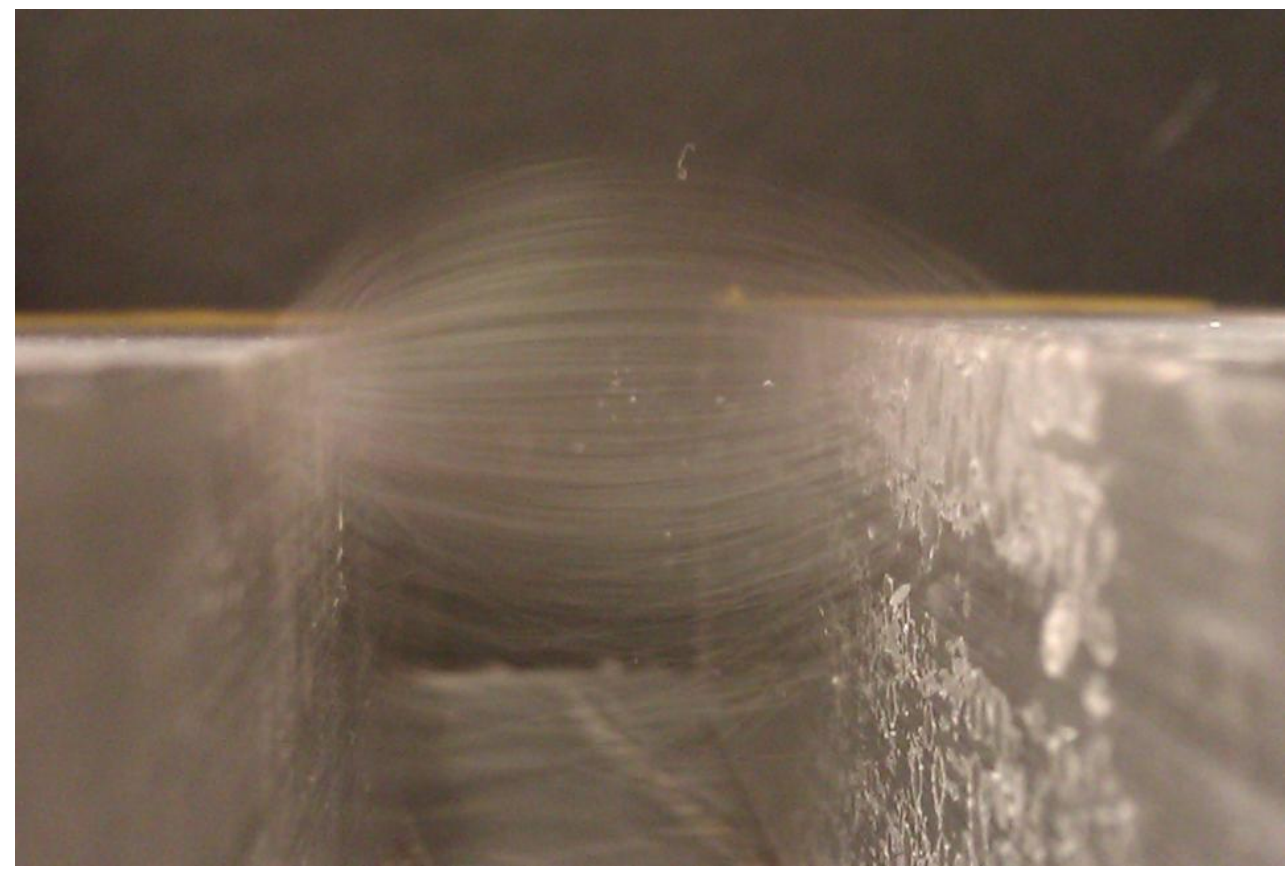

Figure 2.12: Residual charge indicated by arching of deposited nanofibers. These fibers were collected using pure HFIP as the solvent between two aluminum block collectors with a spacing of $2 \mathrm{~cm}$. Note the significant arching in the $Z$ direction. Fibers are being viewed along the $y$ axis as depicted in Figure 2.17. Nanofibers are aligned horizontally between the two aluminum dielectric blocks. Electrospinning needle is located above the sample. 


\subsection{Discussion}

These results yielded several advances over the current state of the art. Firstly, a method to synthesize nanofibers of any diameter was presented. Secondly, the importance of residual charge and stretching electric fields as presented in our group's previous research [63] was thoroughly verified. Thirdly, the required alignment techniques to produce well-aligned nanofibers of a selected size were categorized. Finally, by incorporating the dielectric strength of the PLGA solution, stretching electric fields, and residual electric fields small $<300 \mathrm{~nm}$ highly aligned $\left(A D<15^{\circ}\right)$ nanofiber meshes were synthesized using gap, mandrel, and patterned mandrel alignment techniques.

\subsubsection{Altering fiber diameter}

From these results, one can see that by changing the dielectric strength of the polymer solution we can create smaller nanofibers. The nanofiber diameter and approximate dielectric constant are located in Figure 2.13. As the amount of THF in the electrospinning solution increases, the size of the nanofibers increases, as shown in Table 2.1. A direct correlation between fiber diameter and dielectric constant can be extracted from Figure 2.13. 


\begin{tabular}{|c|c|c|c|c|}
\hline solvent & $\begin{array}{l}\text { Mean fiber } \\
\text { diameter }\end{array}$ & $\begin{array}{l}\text { Reported } \\
\text { dielectric } \\
\text { constant }\end{array}$ & $\begin{array}{l}\text { Dielectric } \\
\text { weighted } \\
\text { average }\end{array}$ & $\begin{array}{c}\text { Conductivity } \\
\left(\mu \mathrm{S} / \mathrm{cm}^{2}\right)\end{array}$ \\
\hline THF & & 7.5 & & \\
\hline DMF & & 38.3 & & \\
\hline 1:1.5 THF:DMF & $100 \mathrm{~nm}$ & & 26.0 & \\
\hline 1:1 THF:DMF & $140 \mathrm{~nm}$ & & 22.9 & 3.9 \\
\hline 2:1 THF:DMF & $300 \mathrm{~nm}$ & & 17.8 & 4.3 \\
\hline 9:1 HFIP:DMF & $500 \mathrm{~nm}$ & & 18.9 & \\
\hline HFIP & $700 \mathrm{~nm}$ & 16.7 & & \\
\hline 3:1 THF:DMF & $700 \mathrm{~nm}$ & & 15.2 & 5.9 \\
\hline 4:1 THF:DMF & $1300 \mathrm{~nm}$ & & 13.7 & \\
\hline \multicolumn{5}{|c|}{$\begin{array}{l}\text { Figure 2.13: Relationship between fiber diameter and dielectric constant of } \\
\text { polymer solution. THF and DMF alone are pure solvents and do not electrospin } \\
\text { fibers well. Concentration of polymer is kept constant at } 20 \% \text {. The estimated } \\
\text { dielectric constants can be seen in the } 4^{\text {th }} \text { column. These numbers are derived by } \\
\text { taking the weighted average of the dielectric constants of pure solvents [82]. }\end{array}$} \\
\hline
\end{tabular}

Increasing the dielectric strength of the polymer solution lowers nanofiber diameter. As more THF was added the resulting fibers increased in size. The vapor pressure of the associated solvents also plays a role in the electrospinning process. Solvents with lower vapor pressure remain in a nanofiber for longer which allows the nanofiber to stretch more. HFIP and THF have similar vapor pressures (120 and 143mmHg respectively), whereas DMF has a vapor pressure of only $2.2 \mathrm{mmHg}$. Therefore HFIP and THF evaporate quickly and DMF evaporates slowly (fibers are spun at room temperature and atmospheric 
pressure). As seen in Figure 2.13, both the higher dielectric strength and lower vapor pressure contribute to smaller nanofibers. Increasing the amount of DMF beyond a critical point will prevent the formation of bead free nanofibers. This is because the polymer remains in solution for too long a time during the electrospinning process. From Figure 2.14 we can correlate fiber diameter with dielectric strength of the solvent. Smaller nanofibers grown with 2:1 THF:DMF and 1:1 THF:DMF required $1 \mathrm{mg} / \mathrm{mL}$ of TEAC to electrospin properly. The exact vapor pressure of a mixture of solvents is very difficult to calculate, as the majority of the solvent THF leaves the fiber during flight because of its high vapor pressure. Further stretching of the nanofiber during electrospinning cannot take place once the solvent has evaporated, as the fiber breaks rather than stretches.

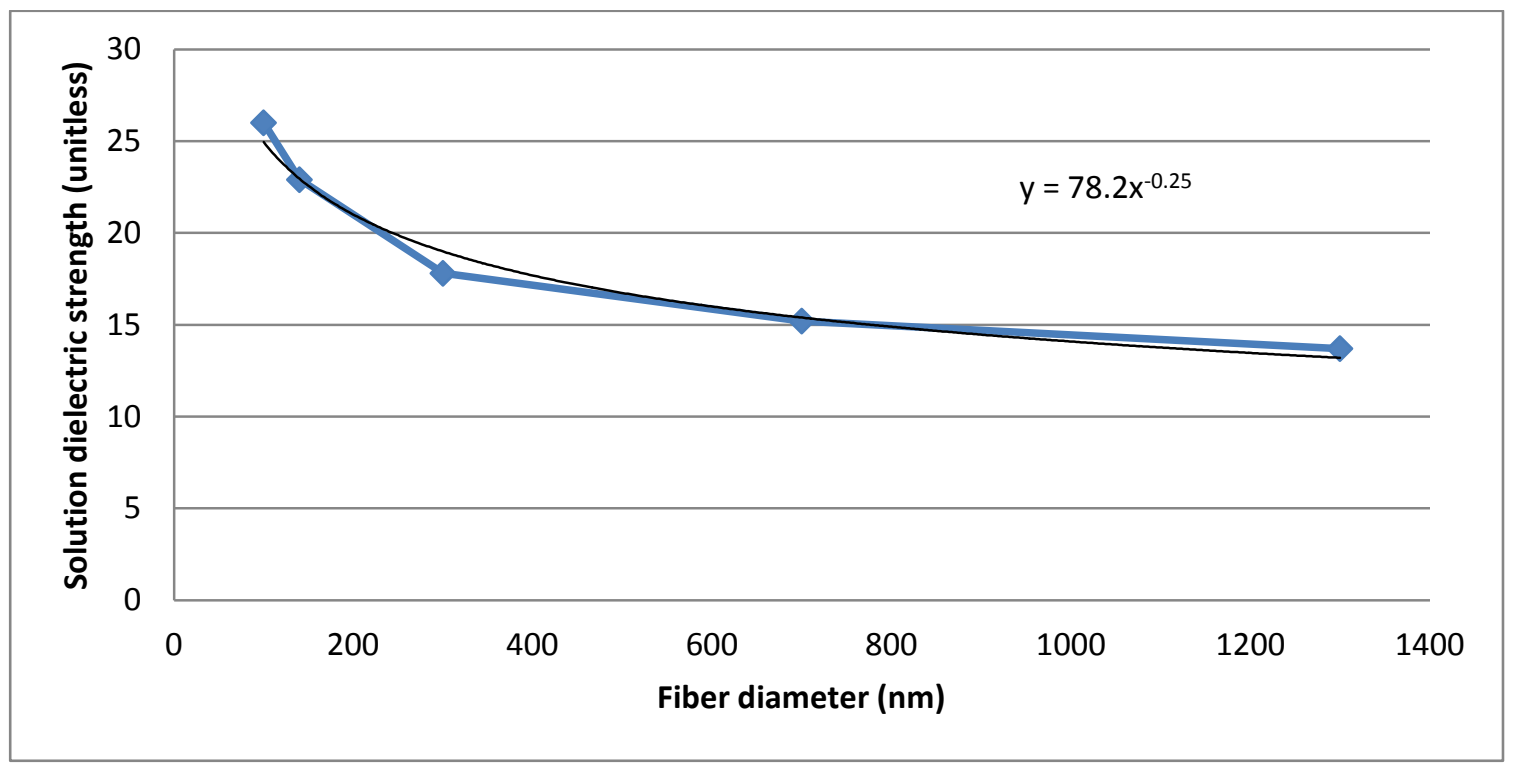

Figure 2.14: PLGA 85:15 fiber diameter vs. solution dielectric strength. A power function provided the best fit line. Graph created from data of electrospun fibers. 
Equation 2.4 can then be used to calculate the required dielectric constant $(\varepsilon)$ of the solvent to achieve the desired fiber diameter (d).

Eq. 2.4

$$
\varepsilon=78.2 * d^{-0.25}
$$

For example, if one wanted 550nm nanofibers the required dielectric strength of the solution is 16.35 . Next, the proper ratio of THF:DMF of the polymer solution with $1 \mathrm{mg} / \mathrm{mL}$ of TEAC is calculated using equation 2.5 .

Eq. 2.5

$$
T H F=-5.167 *\left(d^{0.25}-2.042\right) /\left(d^{0.25}-10.42\right)
$$

From equation 2.5, the required ratio of THF:DMF is 2.48:1. The diameter tailoring process can be applied to additional polymers, provided their viscosities are similar. This was verified by electrospinning 25\% PN GEG PhPh, a polyphosphazene, with 3:1 THF:DMF as seen in Figure 2.15. The higher concentration was used to give the polymer solution a lower viscosity for electrospinning. 


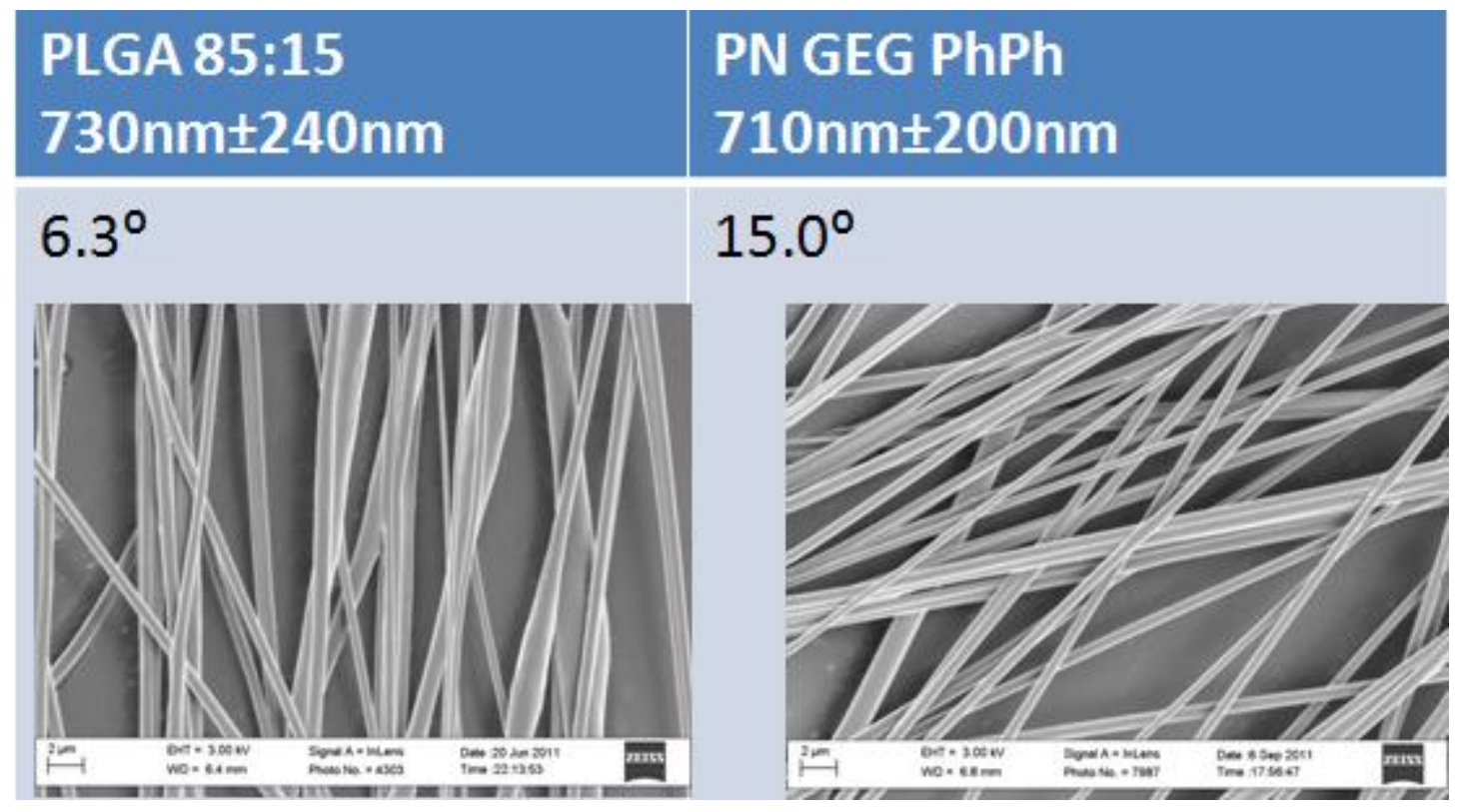

Figure 2.15: comparison between PLGA and blended PN GEG PhPh : PLGA nanofibers. Note the similarities in diameter.

Further discussion of PN GEG PhPh polymer electrospinning can be found in Chapter 3.

\subsubsection{Insulator plate gap}

The gold coated Mylar ${ }^{\mathrm{TM}}$ plate collectors worked well to align smaller nanofibers.

From interpreting our results in Table 2.1, one can see that the smallest nanofibers required a very small gap width to align well. This is directly depicted in Figure 2.16. 


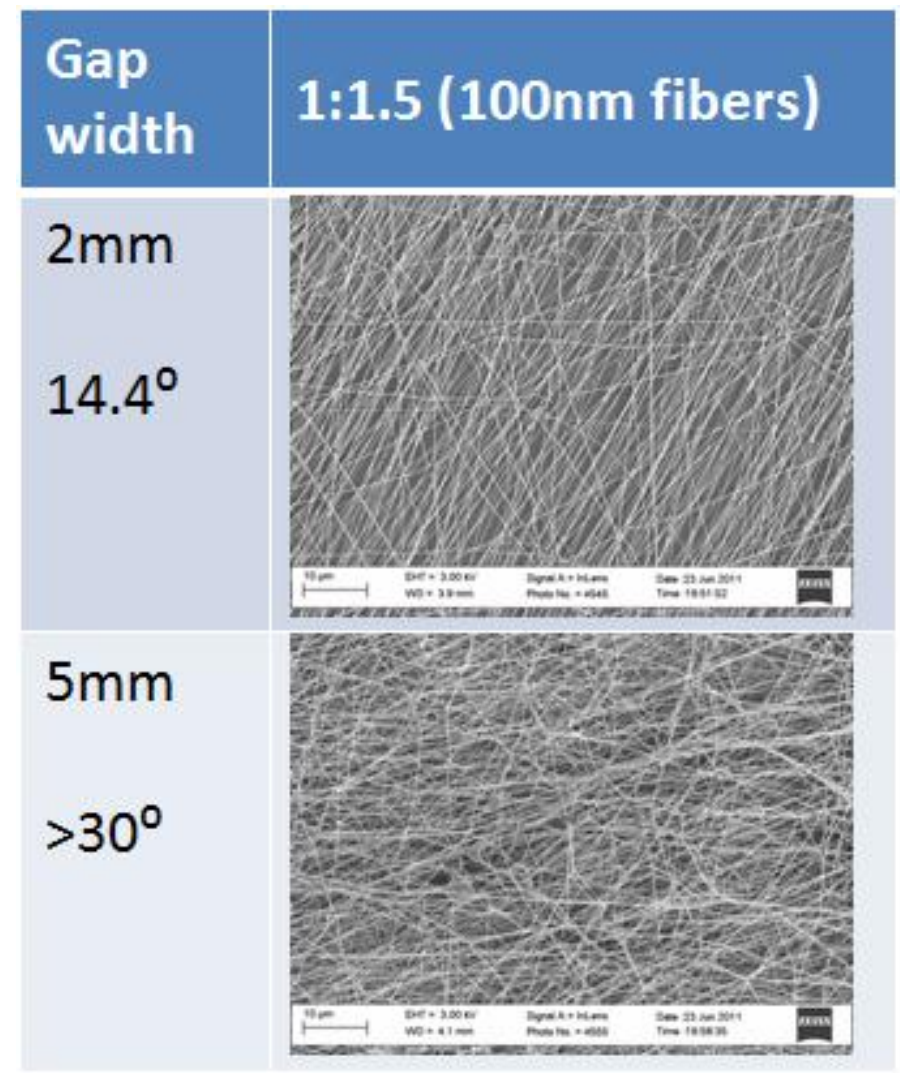

Figure 2.16: small nanofibers require a small gap. Note how distorted the nanofibers become when the insulator gap width increases from $2 \mathrm{~mm}$ to $5 \mathrm{~mm}$.

Understanding the alignment process requires a through discussion of the electric fields involved. Nanofibers stretch when they encounter a patterned dielectric material which influences the charges on the nanofiber. As described in Chapter 1 , when a nanofiber is stretched during the looping and whipping instability phase in electrospinning it is propelled by an attraction of opposite charges. The positively charged solution is attracted toward a source that will supply electrons, in this case the ground. Any patterned dielectric, either dielectric blocks or a metal-on-insulator (such as our patterned Mylar ${ }^{\mathrm{TM}}$ ) collector will apply a stretching force via a charged particle moving in an electric field on the nanofiber while it is in flight. The strength of the stretching electric field on the 
incoming nanofiber is depicted by $E_{x}$ in Figure 2.3. The stretching force is applied over a much greater spatial width for small collectors than for large collectors. This approach allows for more stretching of the fibers near the edges of plate collectors, and has a much stronger effect on nanofiber alignment.

\subsubsection{Mandrel and patterned mandrel}

From the electrospinning results depicted in Figure 2.9, one can see that by tailoring the residual charge on nanofibers, highly aligned $\left(A D<15^{\circ}\right)$ nanofibers of any size can be collected on the mandrel.

Larger $(>400 \mathrm{~nm})$ nanofibers align well on mandrel and patterned mandrel collectors; however, patterned mandrel collectors align nanofibers below 400nm especially well. The reason is that the combination of mechanical alignment through the mandrel as well as electrostatic alignment through a patterned collector provides an additive alignment force. This added attractive force helps "take up the slack" in a growing nanofiber strand, much in the fashion that eyelets along a fishing rod help to prevent the line from getting tangled. Using equations 2.1 and 2.2, the volume and surface area of a cylinder, we know that reducing the radius by $1 / 2$ results in reducing the surface area by $1 / 2$ and reducing the volume by $1 / 4$. If we assume that the mass remains the same (as would be the case with equal electrospinning flow rates), halving the diameter quadruples the length of a nanofiber. For instance, if you have a $200 \mathrm{~nm}$ wide $5 \mathrm{~cm}$ long nanofiber, when you reduce the diameter to $100 \mathrm{~nm}$ the length becomes $20 \mathrm{~cm}$. Physics tells us that force is proportional to both mass and charge, so the 
reduction in mass will not hinder alignment. But because the smaller fibers are so long there is a natural certainty they will get tangled. This continues the fishing rod analogy: a thin line is prone to tangling in large part because one cannot reel in the slack in an organized manner because the thin line will curl more and therefore tangle more. One can combat this phenomenon in electrospinning either by increasing the rotational speed of the mandrel or decreasing the flow rate. Other researchers have placed loops along the path of the nanofibers in hopes to keep the fibers from distorting as they are collected [83]. Growing smaller, aligned nanofibers on mandrel collectors required higher rotational speeds, added charge, and a lower flow rate. Further discussions of future research in electrospinning are presented in Appendix B.

\subsubsection{Dielectric block and residual charge}

As seen in Figure 2.11, the addition of TEAC causes a decrease in nanofiber diameter. Fibers grown with THF:DMF as their solvent with TEAC do not align well on dielectric collectors when the dielectric constant of the electrospinning solution is greater than 17 . The most likely cause is that the stretching force from $E_{x}$ becomes so great that the repulsive force from the deposited nanofibers cannot overpower it to provide alignment. As the THF evaporates and DMF remains, the effective dielectric constant of the solution increases. This loss of THF results in greater opposition to the applied electric field and more stretching. 
Additionally, THF can serve as an electron donor and act to reduce the residual charge left on the deposited nanofibers [84].

Very small fibers could also be distorted from the cooling effect of the THF evaporating during fiber synthesis. As discussed in Chapter 1, when fiber diameter decreases, the volume decreases more quickly than the surface area. Fibers with such small volume may shrivel and distort themselves in response to the massive amount of heat dissipated from THF evaporating from a comparatively large surface area. Further distortion may arise from conservation of kinetic energy: when a molecule of solvent evaporates and leaves to the left, then the fiber is pushed towards the right. Proof of this hypothesis would require a through observation and understanding of the physical chemistry occurring at the molecular level during the electrospinning process, and this domain is far beyond the scope of this dissertation. Nanofibers grown with THF:DMF on insulator gap collectors still align well with the addition of TEAC. All other nanofibers on dielectric blocks with THF:DMF as their primary solvent form misaligned with the addition of TEAC. The reason is that TEAC alone cannot add enough residual charge to align the fibers.

HFIP has none of these shortcomings, and aligns fibers well in all cases as indicated in Figure 2.10. Proof of the residual charge on nanofibers can be seen by the extreme stretching of the deposited fibers in the $Z$ direction in Figure 2.12. The stretching results from the fact that the fibers have retained so many of their charges. Fibers grown with THF:DMF still experience this stretching in the $Z$ direction, although the effect is not so prominent. 
Fibers with less THF and more DMF as their solvent have very high dielectric constants, and therefore experience a greater opposition to applied electric fields. In this case, $E_{x}$ prevents the repulsive electric field $E_{r}$ from effectively acting on the incoming nanofiber. This dominance does not occur with nanofibers with HFIP and TEAC because the HFIP evaporates very evenly which enables uniform nanofiber stretching during the electrospinning process. This leaves a very large $E_{r}$, as evidenced by Figure 2.12. Hence, the proper balance of $E_{x}$ and $E_{r}$ is required to form aligned nanofibers.

\subsection{Effect of magnetic field on nanofibers}

Figures 2.10 and 2.17 examine the effect of a magnetic field on incoming nanofibers. The fact that fibers grown on these two collectors have statistically indistinguishable angular deviations proves that the presence of a magnetic field has no effect on fiber alignment in this situation. 


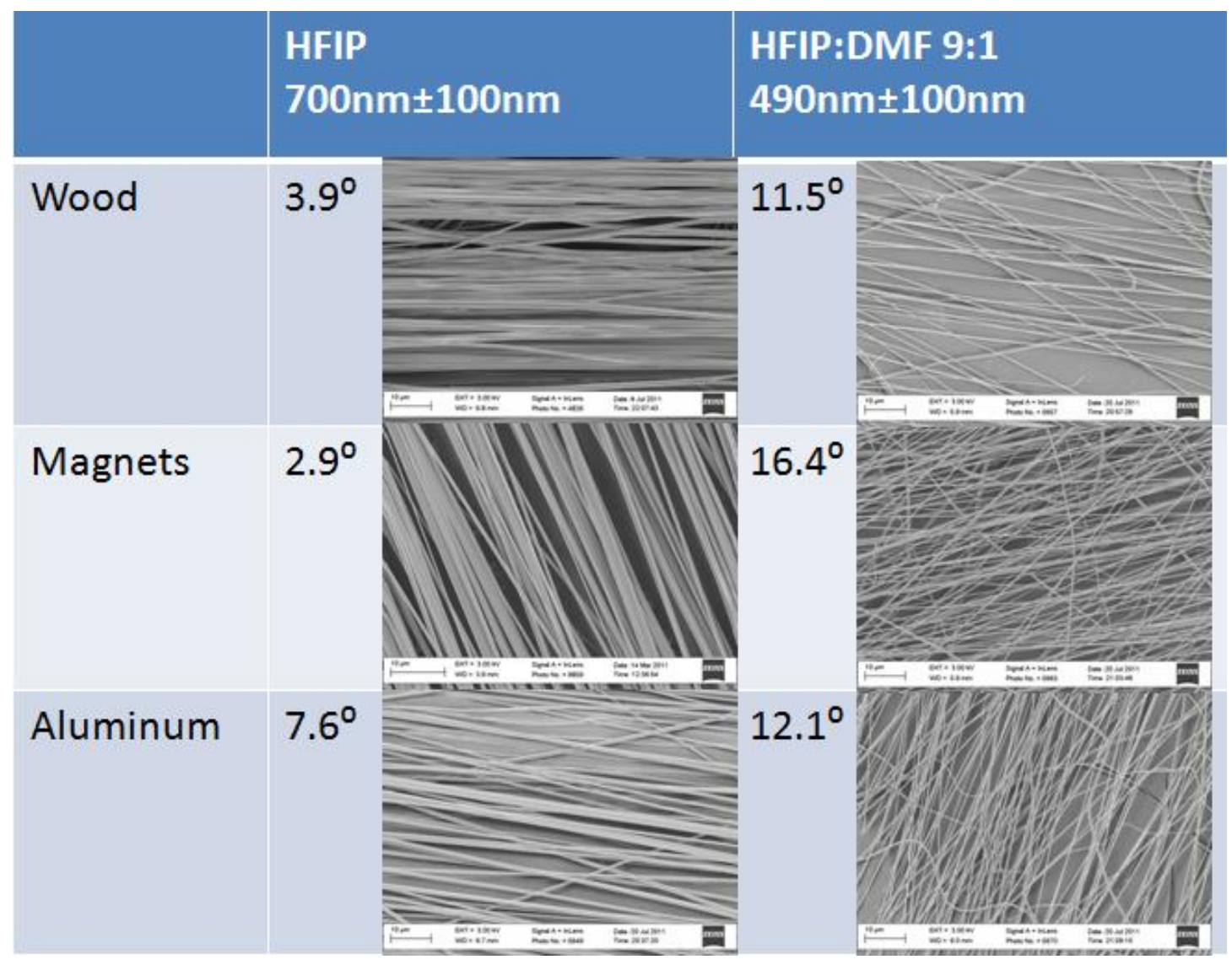

Figure 2.17: Comparison of fibers on dielectric blocks grown with HFIP and with HFIP:DMF 9:1. Note how the tiny addition of DMF drops the diameter yet worsens alignment. Angular deviation is reported in the top left corner. Scale bar is 10 microns.

\subsection{Dielectric alignment of small nanofibers}

To synthesize well-aligned small nanofibers through dielectric gaps one must carefully balance the residual and stretching electric fields. The smallest reproducible nanofibers we were able to synthesize had an angular deviation of $6.8^{\circ}$ and a diameter of $410 \pm 90 \mathrm{~nm}$ when aligned using wooden blocks. Fibers shown in Figure 2.18 


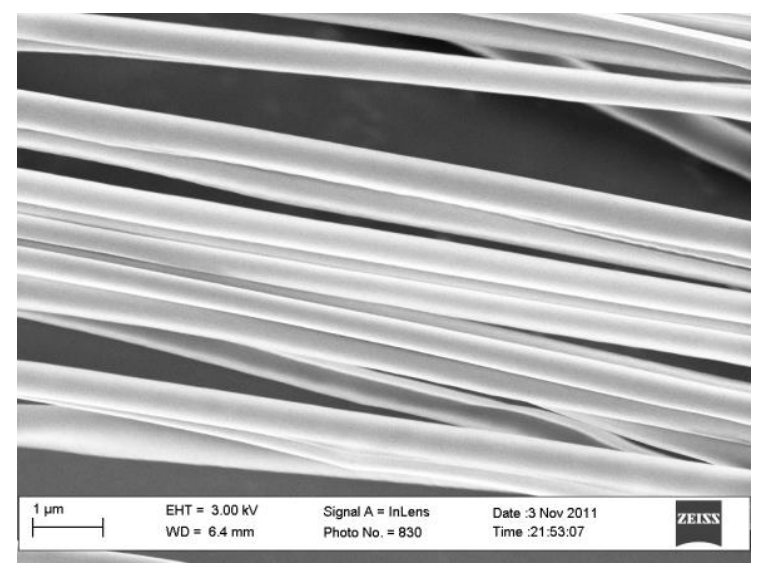

Figure 2.18: Highly aligned smaller nanofibers collected on dielectric wooden blocks with a spacing of $.7 \mathrm{~cm}$. The concentration of polymer in HFIP was lowered to $17 \%$.

\subsection{Conclusions}

From these results we can determine a number of facts about electrospinning. First we can tailor the dielectric strength of our polymer solution to yield the desired fiber diameter. We can also determine the most effective alignment technique for each fiber diameter. Smallest nanofibers aligned the best on plate collectors; medium-sized fibers aligned the best on patterned mandrel, while large fibers aligned the best on the unpatterned mandrel. Mandrel and patterned mandrel techniques can align fibers larger than $400 \mathrm{~nm}$ very well. Dielectric blocks align fibers greater than $400 \mathrm{~nm}$ very well. Fibers with the smallest diameter must be synthesized on plate collectors in order to achieve best alignment. These regimes are depicted in Figure 2.19. 


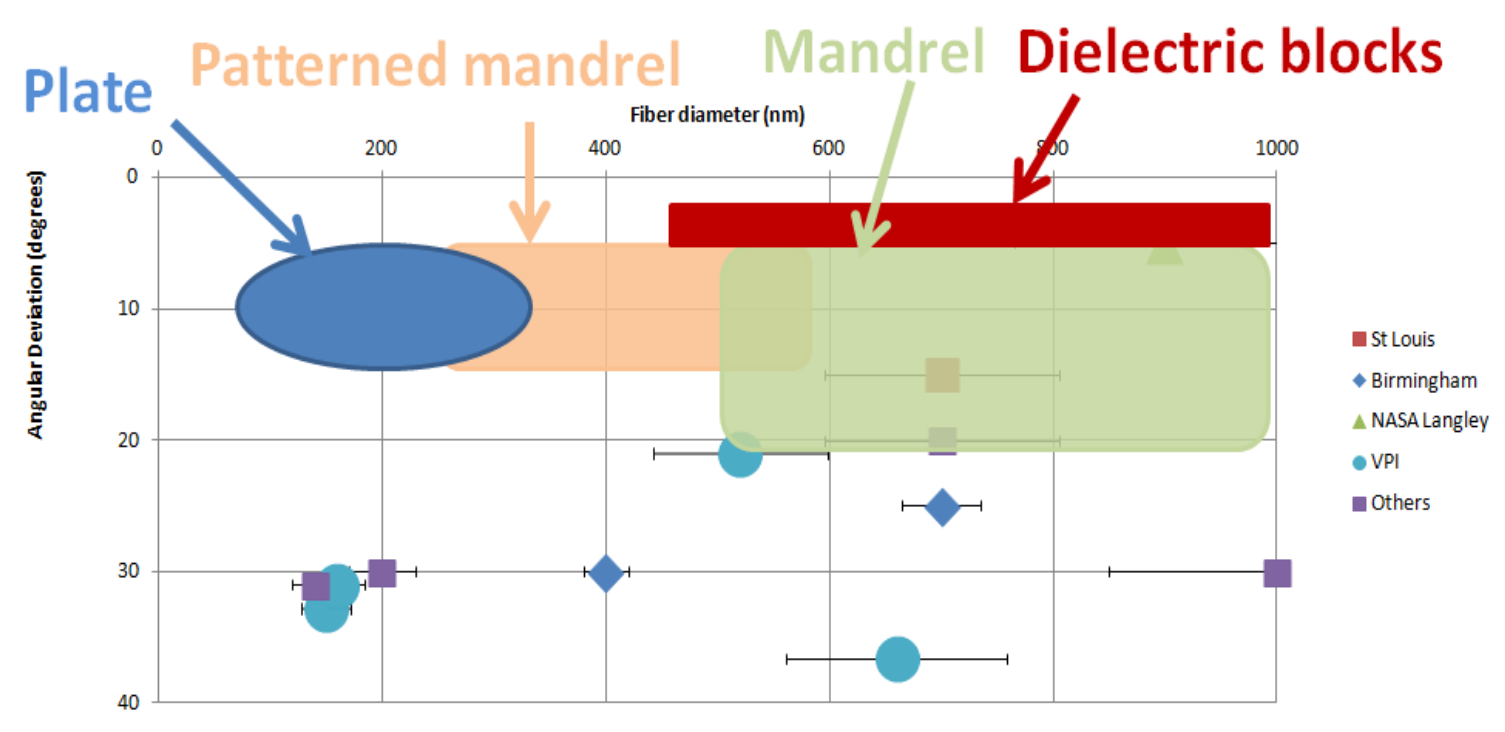

Figure 2.19: Overview of alignment technique required to grow well aligned nanofibers in comparison to researchers at other institutions. Adapted from Figures 2.3, 2.8, 2.9, and 2.14.

Smaller nanofibers require a greater stretching force to align well. This can be easily provided by lowering the gap width in patterned collectors. Once a fiber has been deposited across the low dielectric space, the fiber retains some of the charge it acquired from the high voltage source. This retained charge on each fiber acts to help align each additional fiber as it is deposited across the gap. The nanofibers are packed along in an aligned state because it is the most energetically favorable state.

In conclusion, we have found that fiber diameter is strongly dependent on the dielectric constant of the polymer solution. Using equation 2.4, any diameter nanofiber between about 100 and $1000 \mathrm{~nm}$ can be synthesized. TEAC can be added to fibers to decrease their diameter, regardless of the solvent used in the electrospinning process. Smaller nanofibers require smaller gap widths to align 
because of the increased spatial width over which the stretching electric field is applied in narrower gap collectors. The presence or absence of a magnetic field in the electrospinning process has no effect on the alignment of the nanofibers. Smaller fibers created with solvent dielectric constants greater than 17 do not align well on dielectric blocks regardless of the gap spacing. This misalignment is related to the residual charges present in the electrospun nanofiber mesh. We have also explained the importance of residual charges on an incoming nanofiber layer. By properly balancing all of these attributes, aligned nanofiber meshes of any diameter may be synthesized by tailoring the dielectric constant of the polymer solution and selecting the correct alignment technique as depicted in Figure 2.19.

\subsection{Summary of Chapter 2}

In this chapter we examined the dielectric and electrostatic properties of PLGA $85: 15$ during electrospinning. In the past, there was no systematic way to control fiber diameter. Now we have developed one. This formula allows us to make fibers of different diameters. We also applied our new formula to other polymer systems. The equations to vary nanofiber diameter through dielectric strength of the electrospinning solution will enable future researchers to produce their desired nanofiber diameters. We have expanded the state of the art by using the dielectric properties of the electrospinning solution to yield the desired nanofiber diameter as follows: 
Equation 2.4 can be used to tailor nanofiber diameter. The electrospinning process conditions required are: working distance of $10 \mathrm{~cm}$, voltage of $20 \mathrm{kV}, 21$ gauge needle, $0.3 \mathrm{~mL} / \mathrm{hr}$ flow rate, and $1 \mathrm{mg} / \mathrm{mL}$ TEAC in the polymer solution. The PLGA nanofibers presented in this chapter are smaller and better aligned than any other PLGA nanofibers that have been reported in literature, making future cell work on these nanofibers especially significant.

Finally, other alignment techniques such as mandrel and patterned mandrel collection were evaluated for fiber alignment. Improved nanofiber alignment can be achieved by tailoring the residual charge present in mandrel and patterned mandrel electrospun nanofibers. Finally, discussed in this chapter was dielectric electrospinning. This electrospinning technique was used to explore residual charges in nanofibers. Previous researchers attributed alignment of nanofibers to magnetic effects, but the dielectric collection explanation holds true and even better explains their results. Residual charges on aligned nanofibers play a crucial role in aligning the additional incoming nanofiber layers. 


\section{Chapter 3: polymer charge and ease of alignment}

\subsection{Introduction}

There are currently many polymers and techniques used to create aligned nanostructures $[47,69-72,81]$. These fibers range from highly biocompatible polymers such as collagen, to less compatible polymers such as polyethylene oxide (PEO) or polyvinylpyrrolidone (PVP) [85-86]. Alignment techniques range from using a rotating mandrel, to putting magnetic or conducting nanoparticles in nanofibers to help them align $[18,47,87]$. More promising techniques include: aligning fibers through an insulating layer or aligning them through iron or other particles in the fibers. Certain techniques, such as plate electrospinning, can align polymer sub-groups along the backbone of the polymers [37-38]. This alignment arises not solely because of the electrospinning process, but also because of the orienting electric field lines found between the gaps of plate collectors, as discussed in Chapter 2. For instance, PLGA nanofibers would have oriented $\mathrm{C}=\mathrm{O}$ and $\mathrm{C}-\mathrm{CH}_{3}$ groups when electrospun on plate and dielectric collectors, but not on the mandrel. One would expect there would be slight molecular orientation on patterned mandrel collectors.

The ease of alignment of polymers with subgroups of different sizes and charges has not yet been examined. In this chapter, we examine alignment with different polymer types: PPHOS PN GEG PhPh, PLGA, and PCL, each with a high, medium, or low charge respectively. We examine their alignments on dielectric wooden blocks, patterned gap collectors with different gap sizes, and finally on 
patterned and unpatterned mandrels. Our hypothesis is that with more charge on a polymer backbone, the electrostatic repulsion force between fibers $\left(E_{r}\right)$ will be stronger and the fibers will not align as well. $E_{r}$ will contain not only the residual charge left on nanofibers after electrospinning, but also the inherent charge from the molecular subgroups on the polymer's backbone. This theory is based upon our group's recent publication [63] in which the interplay of charges on nanofibers is discussed. According to this theory, PPHOS will have the best alignment in electrostatic or dielectric collection, followed by PLGA, and finally PCL. All nanofibers will align well on the mandrel, as their expected diameters were around 700nm. More information on these polymers is located in Appendix A. Ours is the first group to examine the effect varying residual charge has on alignment.

\subsection{Materials and Methods}

For this set of experiments, we used polymers with different inherent charges on their backbones and compared their alignment with electrostatic techniques: gap and dielectric collectors versus the mechanical mandrel. The highly charged polymer we will use is poly-glycine ethyl glycinato-phenyl phenoxy-phosphazene (PN GEG PhPh), a type of polyphosphazene (PPHOS). This is a novel polymer with applications in bone tissue engineering [32]. We compared this polyphosphazene to PCL, and PLGA 85:15. PLGA 85:15 nanofibers grown on gap collectors will be our reference sample. PPHOS has a high charge, PLGA 
$85: 15$ has a medium charge, and PCL has a low charge. The size and charge of molecular groups attached to backbones of the polymers give them these different charges. As discussed in Chapter 2, PLGA nanofibers have different angular deviations depending upon fiber size and gap width. We selected a single fiber size of $700 \mathrm{~nm}$ to keep constant throughout this set of experiments. We examined how varying the charge on the polymer backbone and alignment techniques affects the angular deviation of different polymer nanofibers. Nanofibers were aligned using plate gap, dielectric block, and mandrel techniques.

PLGA was electrospun with 3:1 THF:DMF at $20 \%$ weight per volume (milligram/milliliter * 100\%). PPHOS nanofibers were electrospun with 3:1 THF:DMF at $25 \%$ weight per volume blended with low molecular weight PLGA 50:50 [32]. PCL nanofibers were electrospun in THF:DMF as well as DCM at $12 \%$ weight per volume $[29,39]$. These nanofibers were then sputter coated and observed under SEM, and analyzed as described previously in Chapter 2. 


\subsection{Results and Discussion}

PLGA, GEG PhPh, and PCL aligned nanofibers were electrospun on dielectric wooden block collectors spaced at $1 \mathrm{~cm}$ as shown here.

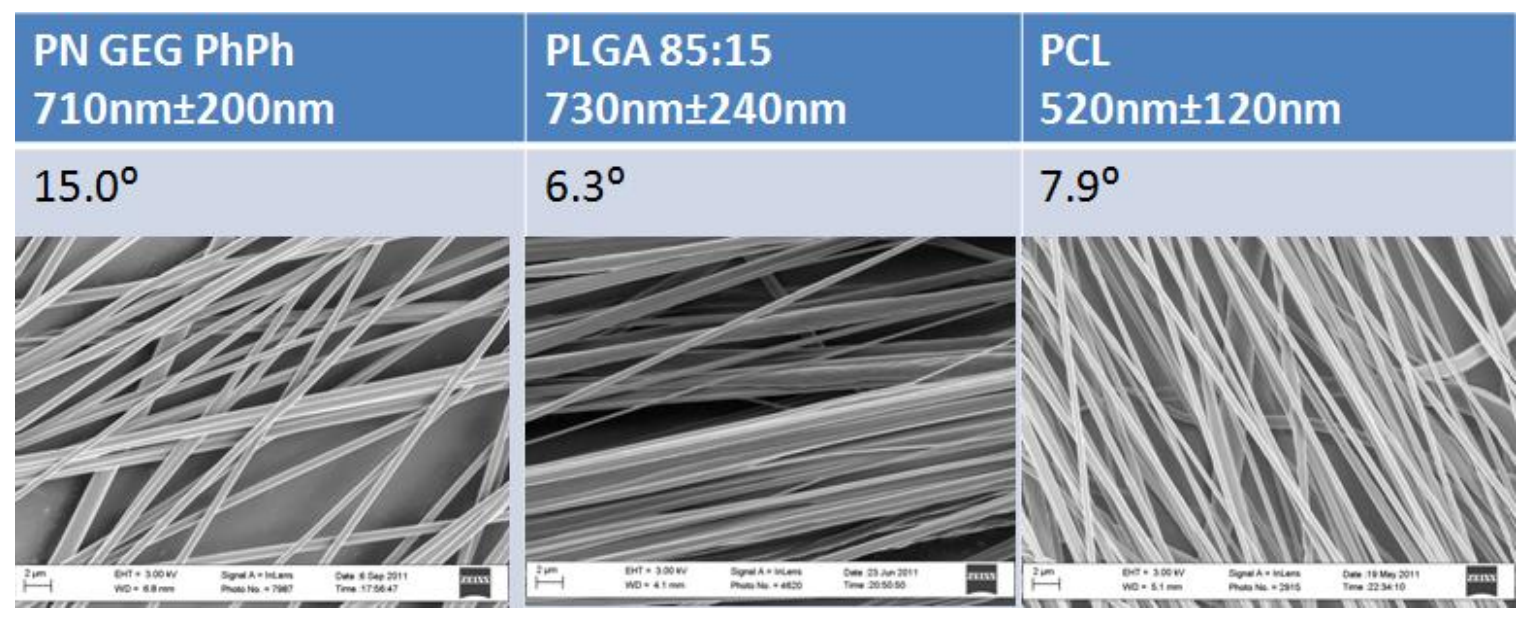

Figure 3.1: PN GEG PhPh, PLGA, and PCL electron fibers on dielectric block collectors.

In Figure 3.1 we see good alignment of PLGA but only fair alignment of PN GEG PhPh. PCL aligned well as expected. PN GEG PhPh is a highly charged polymer, with lots of charge groups on the polymer backbone. These charges on the polymer backbone increased the residual charge, as seen in Figure 3.2 to prevent $E_{x}$ from acting over enough of the spatial width of the dielectric block collector, necessary to yield well-aligned the nanofibers. Therefore, there exists a minimum spatial width over which Ex must exert a strong enough force on the nanofiber to align it. 


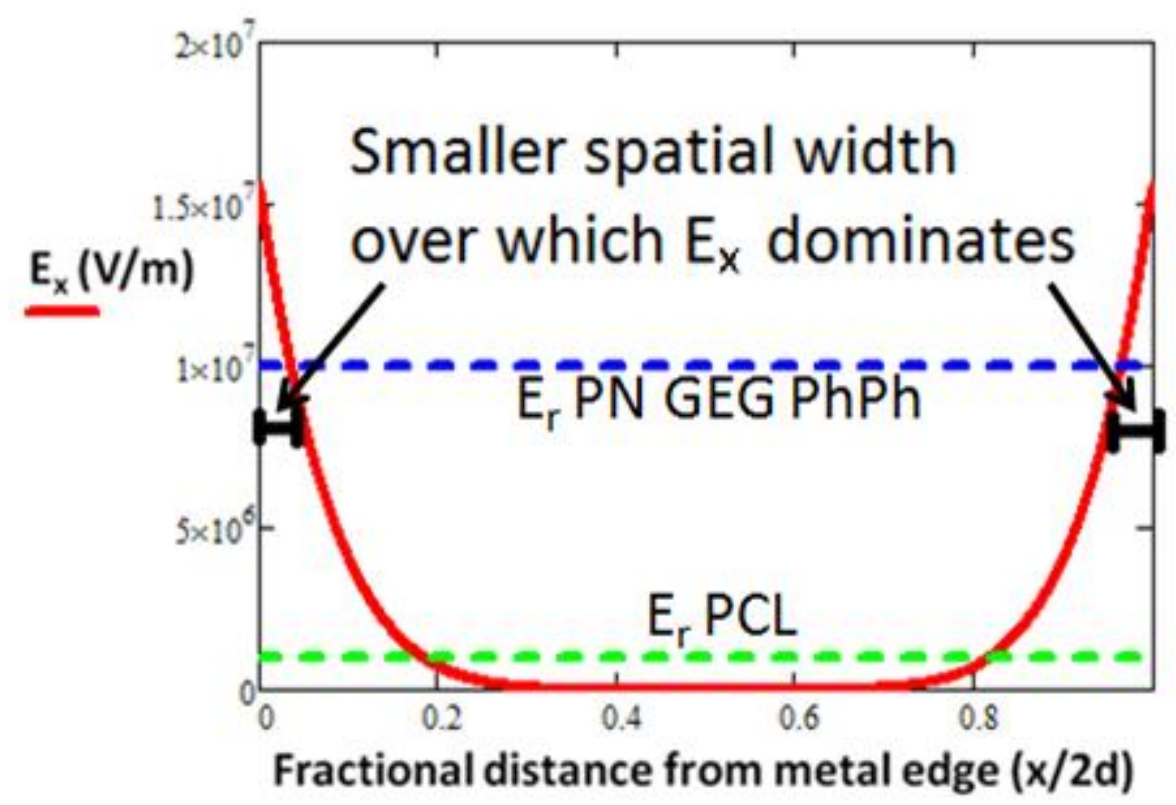

Figure 3.2: Change in residual charges for PN GEG PhPh and PCL nanofibers on dielectric block collectors. The charge on PLGA nanofibers in this case lies between the $E_{r}$ for PCL and PN GEG PhPh. It is omitted for clarity. Model and figure adapted from [63] and curve truncated to better illustrate interactions between the fields.

Next, $E_{x}$ was increased by using patterned Mylar ${ }^{\mathrm{TM}}$ collectors instead of dielectric block collectors.

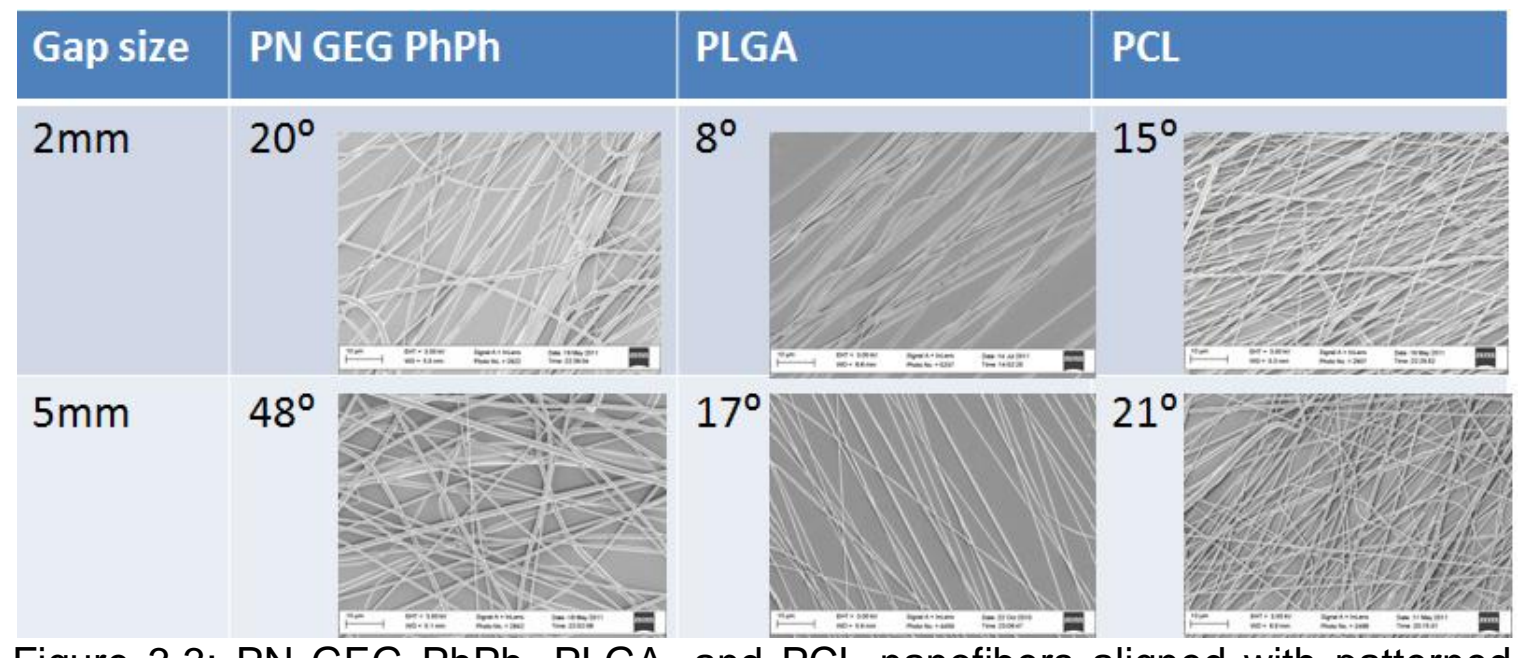

Figure 3.3: PN GEG PhPh, PLGA, and PCL nanofibers aligned with patterned 
Mylar ${ }^{\mathrm{TM}}$ collectors of different gap widths. Note the poor alignment of PN GEG $\mathrm{PhPh}$ and PCL.

In this experiment, the stronger $E_{x}$ actually damaged the alignment of PCL nanofibers because the fibers did not exhibit enough residual charge over a large enough spatial width to form a highly aligned mesh. Strengthening of $E_{x}$ by using patterned Mylar ${ }^{\mathrm{TM}}$ collectors sharpens the profile of the electric field. The lowcharged PCL did not have enough residual charge to align incoming fibers. Fibers were simply stretched by $E_{x}$ and deposited on the collector.

PN GEG PhPh nanofibers did not align well in this case again because the high residual charge still prevented the stretching electric field from sufficiently acting on the fibers as they were deposited. Our model correctly predicted that the highly charged fibers aligned better on smaller gap widths $(2 \mathrm{~mm})$ than larger (5mm) gaps, and that decreasing the gap size in patterned Mylar ${ }^{\mathrm{TM}}$ collectors improves alignment. This model was not able to explain the increase in angular deviation of nanofibers when switching from dielectric block collectors to patterned Mylar ${ }^{\mathrm{TM}}$ collectors. We expected to find that as gap width decreased from dielectric block collectors to patterned Mylar ${ }^{\mathrm{TM}}$ gap collectors alignment would improve. Instead, we found that alignment for PN GEG PhPh on Mylar ${ }^{\mathrm{TM}}$ collectors was worse than it was for patterned dielectric block. The most likely explanation is the differences in alignment techniques. Patterned Mylar ${ }^{\mathrm{TM}}$ collectors have a very shallow collector depth (tens of nanometers of gold deposited on Mylar ${ }^{\mathrm{TM}}$ film) whereas dielectric block collectors have a depth of 
$2.5 \mathrm{~cm}$. Future research is needed to incorporate collector depth into our model. Our model correctly predicted that to improve alignment of nanofibers smaller gap sizes are needed.

All the tested polymers electrospun and aligned very well $\left(A D<10^{\circ}\right)$ using mechanical alignment techniques. This experiment proved that residual charges on nanofibers play a major role in electrostatic or dielectric alignment, and that large diameter nanofibers with large residual charges can still align well through mechanical alignment techniques.

\subsection{Conclusions}

We have examined the effect of polymers with high and low charge density of the aligned with different techniques. Our earlier theory on residual and stretching electric fields had to be modified in part, because it did not explain why alignment was not improved in the transition from $1 \mathrm{~cm}$ dielectric block to $0.5 \mathrm{~cm}$ gap patterned Mylar ${ }^{\mathrm{TM}}$ collectors. We have shown that the patterned Mylar ${ }^{\mathrm{TM}}$ collectors do not align fibers with very high or very low charge. Our model predicted that polymers with high residual charge would align well. PN GEG $\mathrm{PhPh}$ has such a high charge it is able to overcome the stretching electric field and collect misaligned. PCL nanofibers also do not align well because of their lack of strong residual charge. Our results on dielectric blocks show us that if the charge density of a polymer is too high, the nanofibers will not align well. 


\subsection{Summary of Chapter 3}

In this chapter we discussed the behavior of polymers with different charges. The charge density on the fibers is related to the molecular structure of the polymer. We examined fibers with high, medium, and low charge, and determined that fibers with high charge do not align well with electrostatic alignment techniques. We also discussed the ability of the residual charges on aligned fibers to improve the alignment of any incoming nanofibers. Polymers with too low or too high residual charge cannot align well on patterned Mylar ${ }^{\mathrm{TM}}$ collectors because the stretching electric field is too insignificant or too dominant over the field from residual charge. This work also led to modification of the theory about the stretching and residual electric fields because dielectric block collectors did not follow the current model. Future models will incorporate the transition from dielectric block collectors to patterned Mylar ${ }^{\mathrm{TM}}$ collectors. Without residual charge, nanofibers do not align. This work is the first which examines nanofiber alignment by directly varying the charge on the polymer backbones. 


\section{Chapter 4: Enhancing cell adhesion and alignment through the use of aligned PLGA nanofibers}

\subsection{Abstract}

In this chapter, we will utilize the aligned nanofibers of varying size grown with alignment techniques discussed in Chapter 2 to modulate cell behavior. There are no reports of synthesized nanofibers of successively smaller size range and angular deviations $<15^{\circ}$ utilized to examine cellular response to these fibers, therefore this series of experiments will advance the state of the art research in this area. Although it is widely accepted that molecular orientation exists in electrospun nanofibers [37-38,76-80], cellular response to molecular level orientation has not yet been thoroughly documented [46,76-80]. Molecular orientation is a localized high degree of charged dipole orientation along the geometric alignment axis of the nanofiber. This molecular orientation forms a uniform distribution of charges along the polymer surface charges thereby reducing repulsive interactions of the molecular subgroups within the functional groups of the polymer [76-80]. Our previous work has shown that NIH-3T3 cells will align better on electrostatically aligned nanofiber meshes with oriented subgroups than on mandrel or patterned mandrel aligned nanofibers. Therefore, we are going to study the effect of oriented molecular subgroups on Schwann cell morphology.

This work advances the current state of the art by determining which diameter nanofibers will best align Schwann and PC12 cells, and what effect molecular 
orientation of polymer subgroups has on Schwann cells. PC12 cells will be cultured on poly-d,I-lysine coated nanofibers to determine the optimal fiber diameter for maximal PC12 neurite extension. This coating is required to assist in cellular adhesion [8].

\subsection{Fibroblasts on molecularly oriented nanofibers}

Our previous research on NIH-3T3 fibroblasts has shown that mechanically aligned larger nanofibers align fibroblasts more effectively than smaller nanofibers, because fibroblasts will stop elongating when they encounter a misaligned smaller nanofiber, as evidenced in Figures 4.1 and 4.2. This shows that fibers of smaller size must be very highly aligned when compared to large fibers with similar alignment to provide equal cell directionality cues. These figures suggest that on larger fibers the lamellipodia follows one or two fibers, while on smaller fibers the lamellipodia no longer continues along the small nanofibers once the filopodia encounter a misaligned nanofiber. 


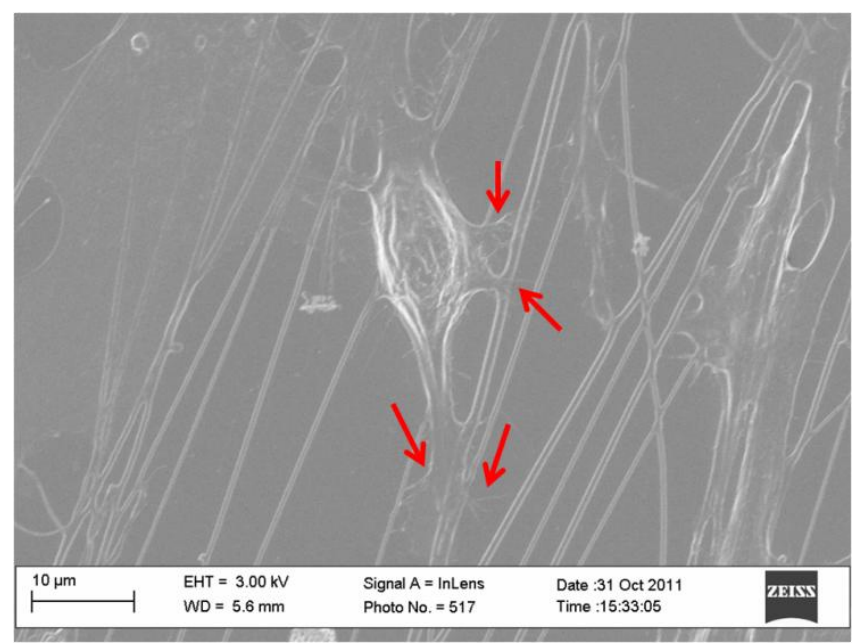

Figure 4.1: NIH-3T3 fibroblast on aligned $740 \pm 280 \mathrm{~nm}$ nanofibers. Note the filopodia indicated by the arrows.

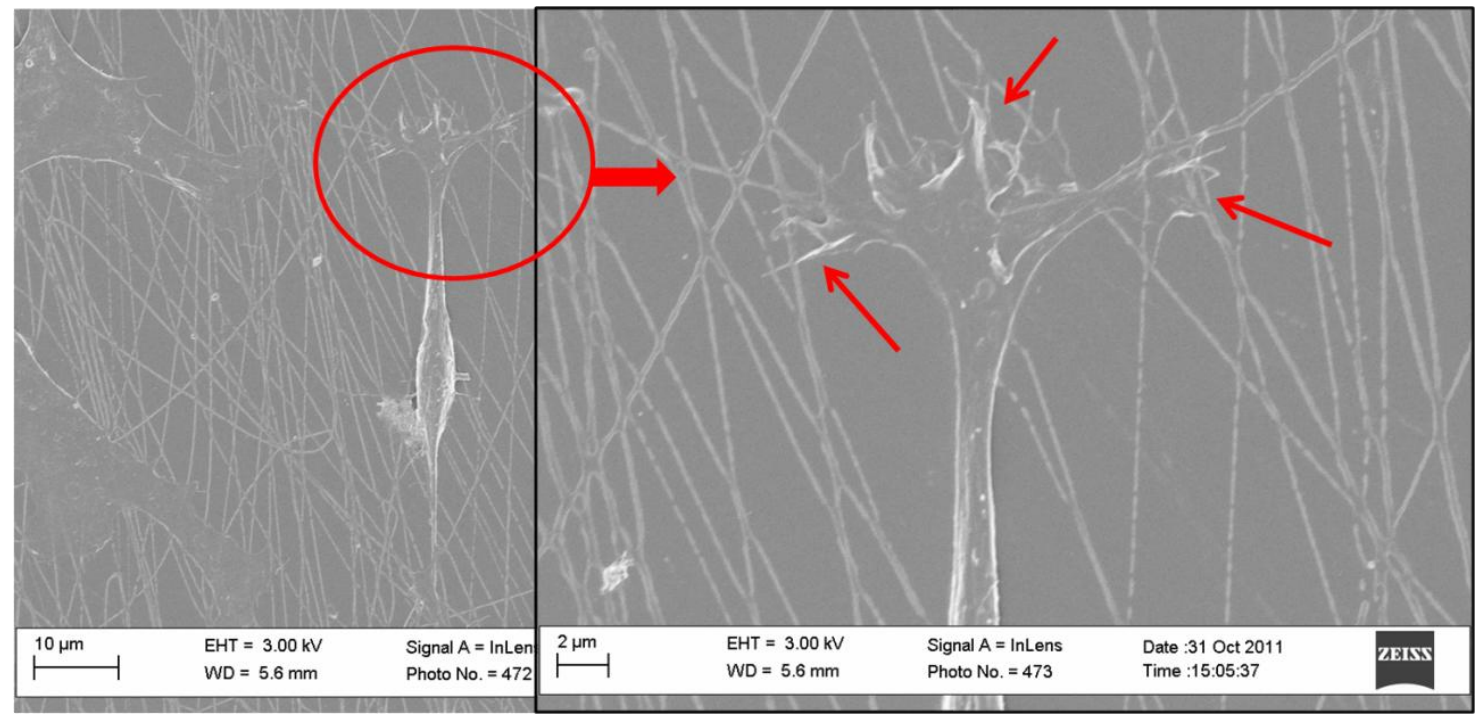

Figure 4.2: NIH-3T3 fibroblast on aligned $140 \pm 40 \mathrm{~nm}$ nanofibers. Note the filopodia indicated by the arrows and cessation of elongation once the cell encounters the horizontally misaligned nanofiber.

Previous research has also shown that depending upon which fiber alignment technique was utilized, nanofibers may have aligned subgroups within their polymer backbones [76-80]. Our previous research has also shown cells favor 
plate aligned nanofibers over unpatterned and patterned mandrel techniques. The reason is that the molecularly oriented subgroups on the nanofibers can cause highly uniform surface chemistries on the surface of the nanofiber, making these oriented hot-spots ideal for the adsorption of adhesion proteins [46].

\subsection{Schwann cell interaction with highly aligned, molecularly oriented nanofibers of varying diameter}

There have been many previous studies on the influence of topographic cues to Schwann cell behavior and nerve fiber alignment [25,88-89]. Many of these studies used aligned nanofibers as a substitute for the ECM. Previous work has been performed on a range of larger aligned fibers, from $200 \mu \mathrm{m}$ to $1.3 \mu \mathrm{m}$ in diameter [25,90]. Results from these studies indicated that the $1.3 \mu \mathrm{m}$ fibers produce better aligned Schwann cells [90]. Research performed on fibers less than a micron either have poor angular deviations of 19.5 degrees or worse [69] or poor control of fiber diameter (variation of $\pm 50 \%$ of average diameter) $[69,91$ 92]. There exists a major shortcoming in the literature: no study has evaluated Schwann cells on well aligned $(A D<15)$ nanofibers of successively smaller sizes, nor has any study examined the effects of molecular level orientation on Schwann cell area, alignment, or elongation. 


\subsubsection{Schwann cell Materials and Methods}

PLGA 85:15 nanofibers were synthesized as described in Chapter 2. Schwann cells were obtained from the University of Virginia Tissue Culture Facility and maintained in DMEM with 10\% FBS and 1\% pen-strep. Penicillin-streptomycin was added to prevent bacterial growth. Cells were seeded on $1 \mathrm{~cm}^{2}$ nanofiber samples of similar alignments at a density of 2500 cells $/ \mathrm{cm}^{2}$. Nanofiber density, or number of fibers per unit area, was kept constant between the samples. Further information on our Schwann cell culture procedures can be found in Appendix C. Three sets of fiber samples (patterned Mylar ${ }^{\mathrm{TM}}$ gap, mandrel, patterned mandrel) were used for Schwann cell evaluation with three different diameters of $740 \pm 280 \mathrm{~nm}, 245 \pm 85 \mathrm{~nm}$, and $140 \pm 40 \mathrm{~nm}$ (nine samples total). Fibers were grown using patterned Mylar $^{\mathrm{TM}}$ electrostatic plate gap, mandrel, and patterned mandrel alignment techniques. Three nanofiber samples consisting of large and small plate aligned nanofibers and a large mandrel fiber sample were coated with poly-d,I-lysine to block any molecular level orientation sites on plate nanofiber samples. This procedure was done to rule out the size effects versus molecular orientation of underlying nanofibers. These samples were compared to uncoated fibers to observe any cell morphological differences. Schwann cells were fixed, stained for f-actin, and imaged. Images were analyzed for cell area, alignment, and elongation using ImageJ. Detailed procedures can be found in Appendix C. Multiple runs of each sample were performed, and over 1000 Schwann cells were analyzed per sample point. For this experiment, we will use 
the null hypothesis assuming all nanofiber samples will produce statistically indistinguishable cell area, alignment, and elongation.

\subsubsection{Schwann cell Results and Discussion}

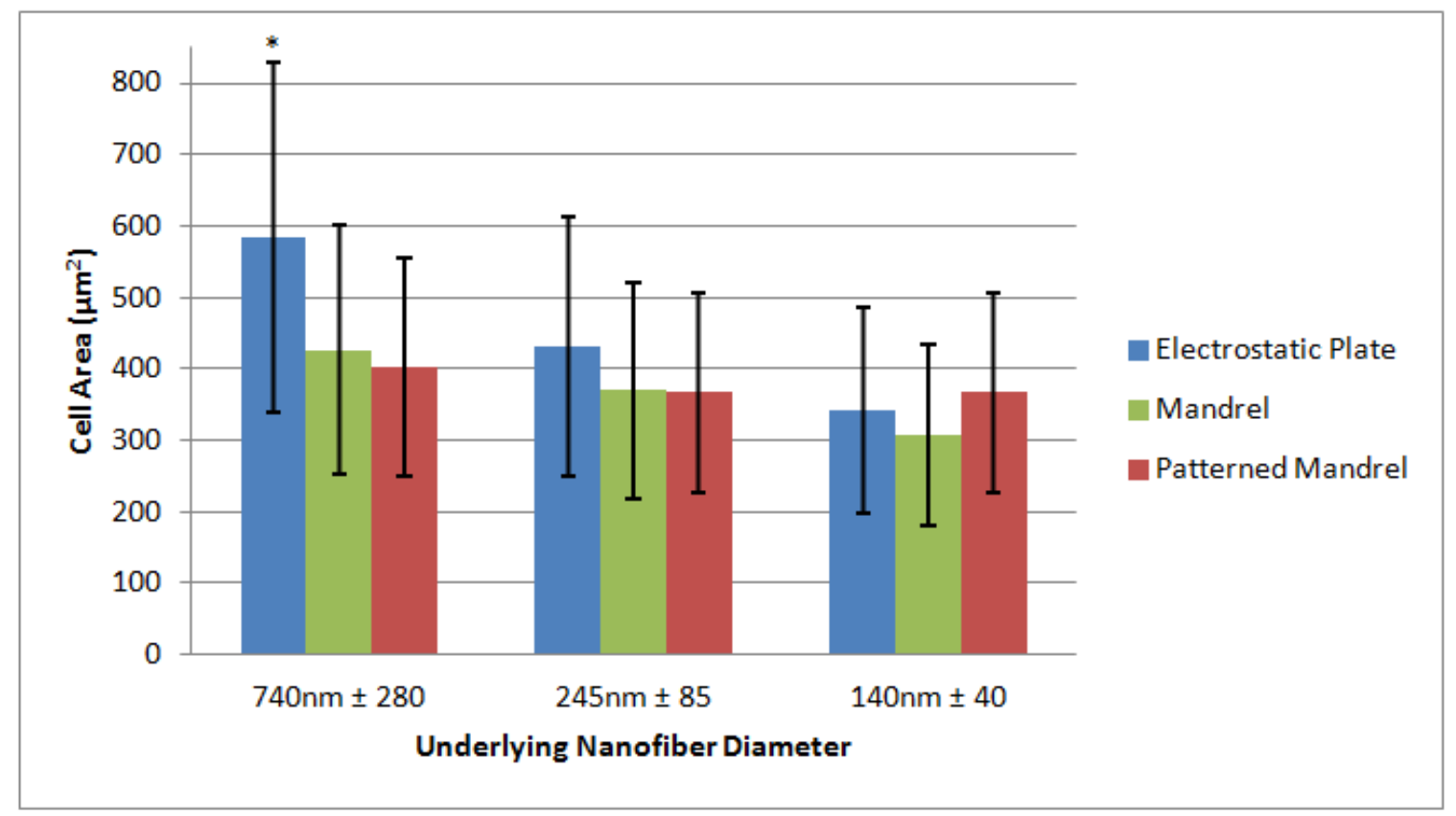

Figure 4.3: Schwann cell area on aligned uncoated nanofibers. Note underlying fiber alignment was held at $10^{\circ} \pm 3^{\circ}$ and fiber density was kept constant for each diameter range. Note the very high cell area in large plate fibers. ${ }^{*}$ is statistically significant with $\mathrm{P}<.01$. 
No statistically significant difference was observed between the mandrel and patterned mandrel samples. This was in disagreement with our null hypothesis. Cells only experienced large spreading on the largest electrostatically aligned nanofiber samples. Cell major length was around 52 microns for each sample, except it was slightly shorter $(33 \mu \mathrm{m})$ for the smallest mandrel nanofiber sample. Cell elongation (major axis/minor axis) was at least 5.9 and statistically indistinguishable in all cases, except for the smallest mandrel aligned nanofiber sample.

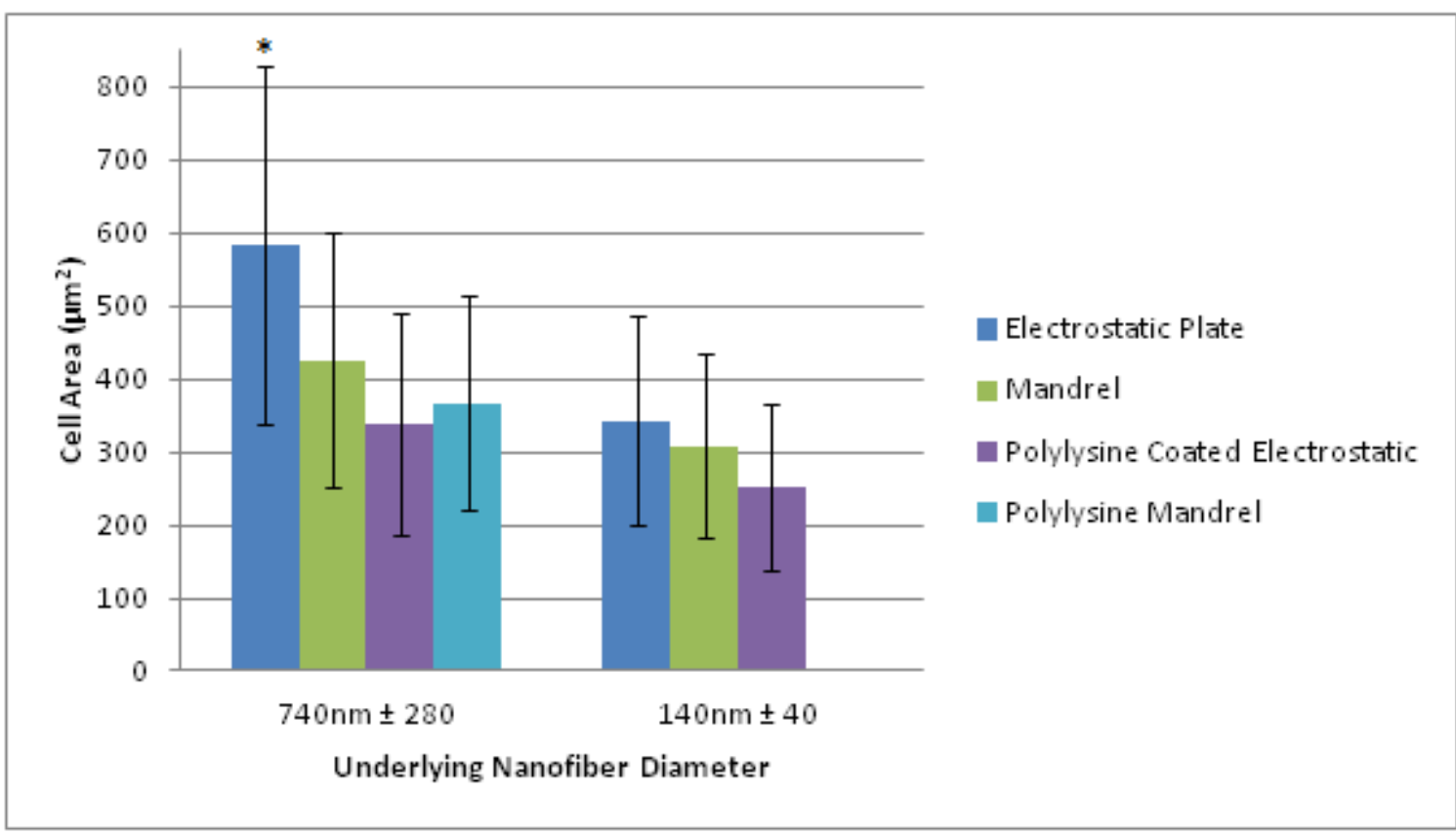

Figure 4.4: Differences between poly-D,L-lysine coated fibers and original uncoated fibers. Note the decrease in area in all samples and the decrease in length of the major axis in the smallest fibers. Note the decrease in area in all samples, but smaller decrease in the large mandrel samples. ${ }^{*}$ is statistically significant with $\mathrm{P}<.01$. 
Adding the polylysine coating had no effect on the major lengths of the cells, their elongation, or their directionality. Adding the polylysine only reduced the cell area, bringing it in line with other similarly sized samples. Coating the electrostatically aligned plate nanofibers covered up the molecularly oriented hot spots, thus lowering cell area. Adding the coating had no effect on the cell major length on the largest nanofibers which remained around 52 microns, but Schwann cells on the smallest nanofiber samples had their lengths reduced to 43 microns. Cell elongation (major axis/minor axis) improved slightly to 6.5. This finding is in line with the concept that polylysine removes any molecularly oriented zones, as the fiber area decreases. Cells adhere well and therefore elongate more on polylysine coated nanofiber samples. 


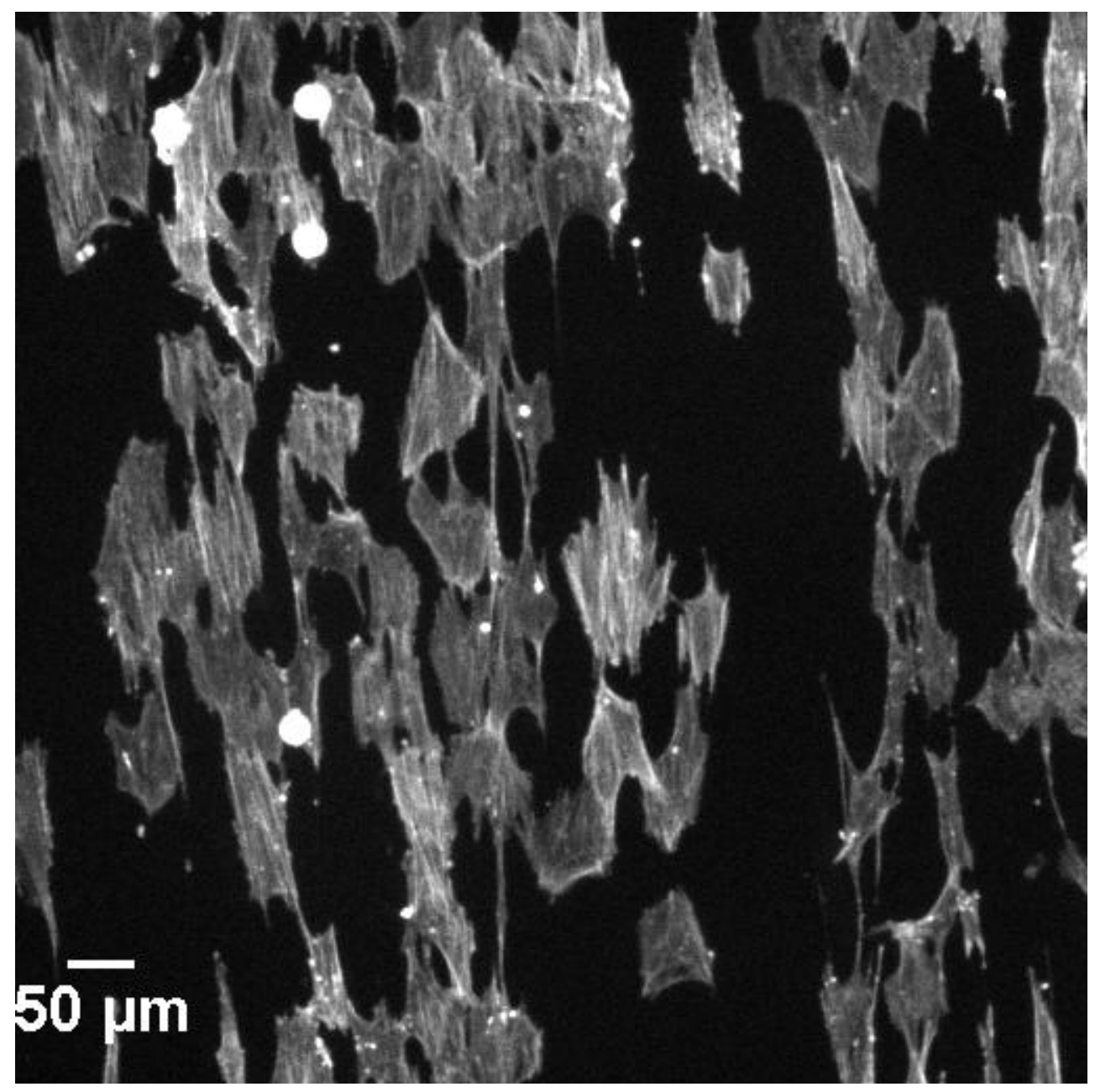

Figure 4.5: Schwann cells arranged as bands of Büngner on large $(740 \mathrm{~nm})$ plate aligned fibers. 


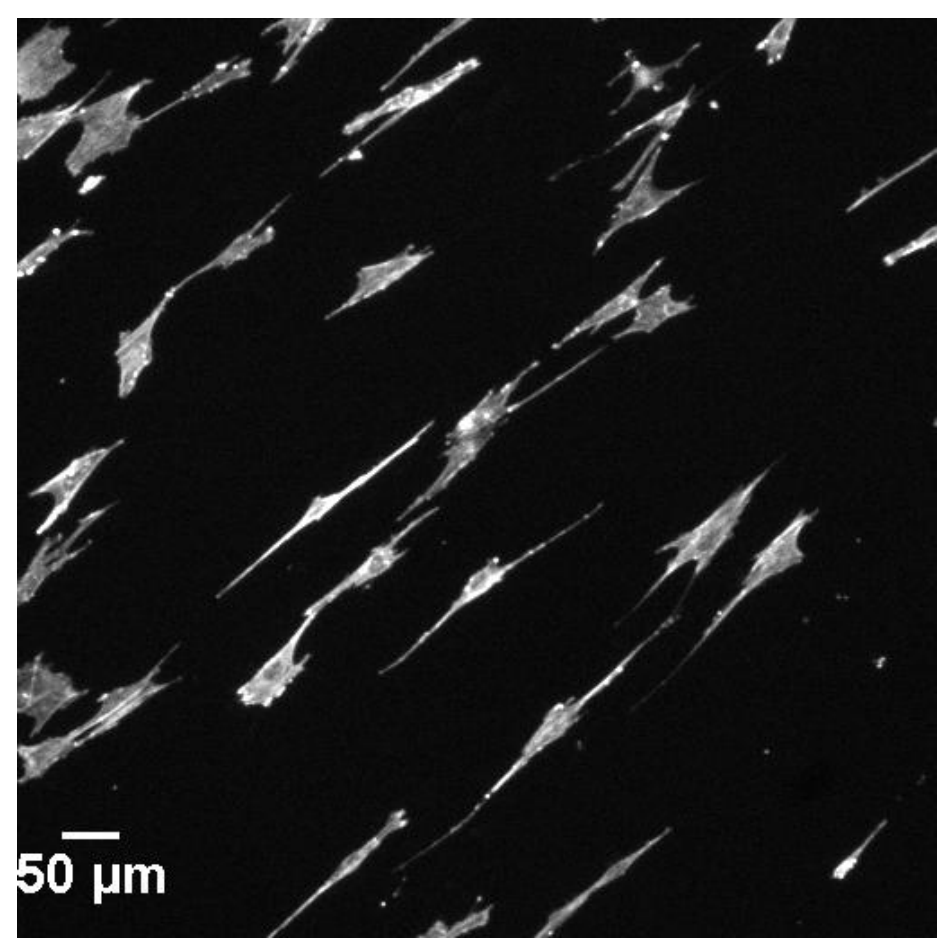

Figure 4.6: Schwann cells on large mandrel fibers. Note the significant cell alignment and elongation.

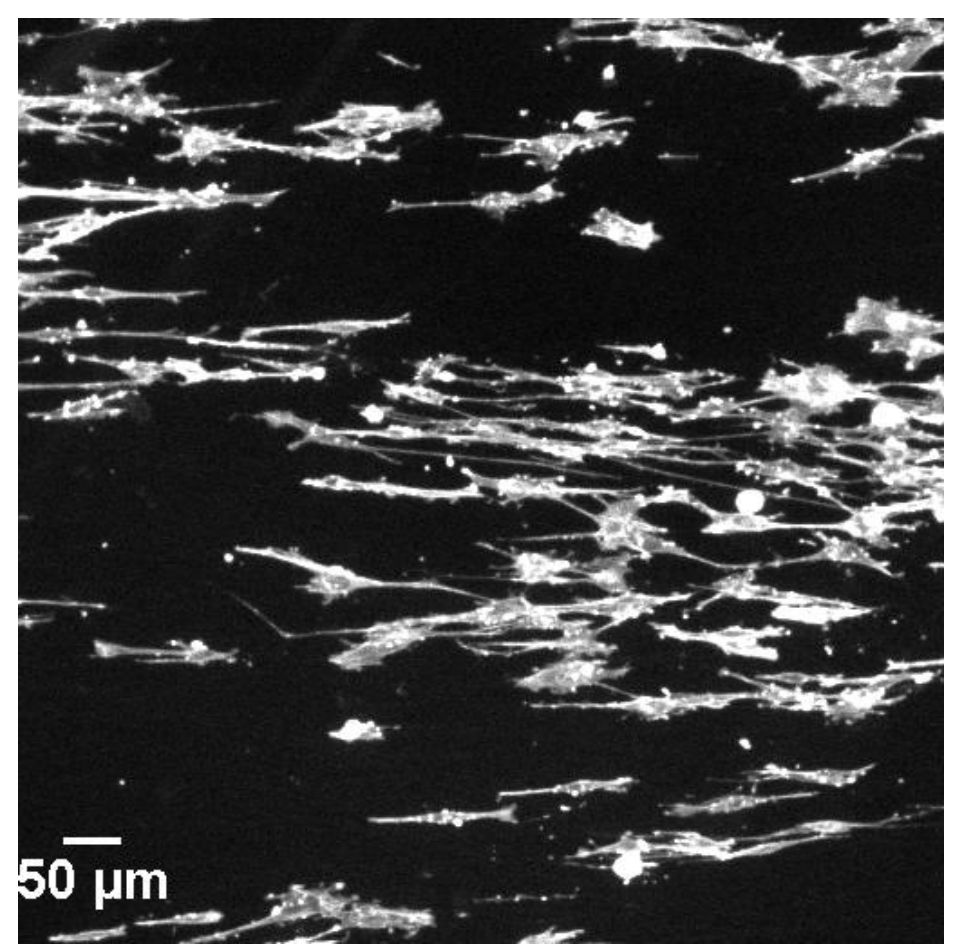

Figure 4.7: Schwann cells on large poly-D,L-lysine coated 740nm plate nanofibers. Note the decrease in area of the cells from Figure 4.5. 
These results, as seen in Figure 4.3, show that Schwann cells exhibited drastically different cell spreading on the largest plate nanofibers as compared to other nanofiber sizes and alignment techniques. The Schwann cell area was almost twice as large on the large plate fibers compared to all other fibers, thus disproving the null hypothesis. Molecular level alignment of the polymer groups in the plate aligned samples explains this phenomenon $[27,37-38,76-80]$. Cells were able to find additional good adhesion sites for attachment. Figure 4.4 shows that by coating the fibers with poly-D,L-lysine we are able to block these molecularly oriented adhesion sites and reduce cell spreading. More cell spreading in Schwann cells is ideal, because the next step in peripheral nerve regeneration is for the Schwann cells to myelinate an axon as it reinnervates its target [93, regen boil medicine]. When Schwann cells prepare to remyelinate a damaged neuron, they form bands of Büngner. These bands are indicated by increased Schwann cell area, alignment of the Schwann cells, alignment of Schwann cell nuclei, and the increased presence of the cell adhesion molecule L1 [93]. 


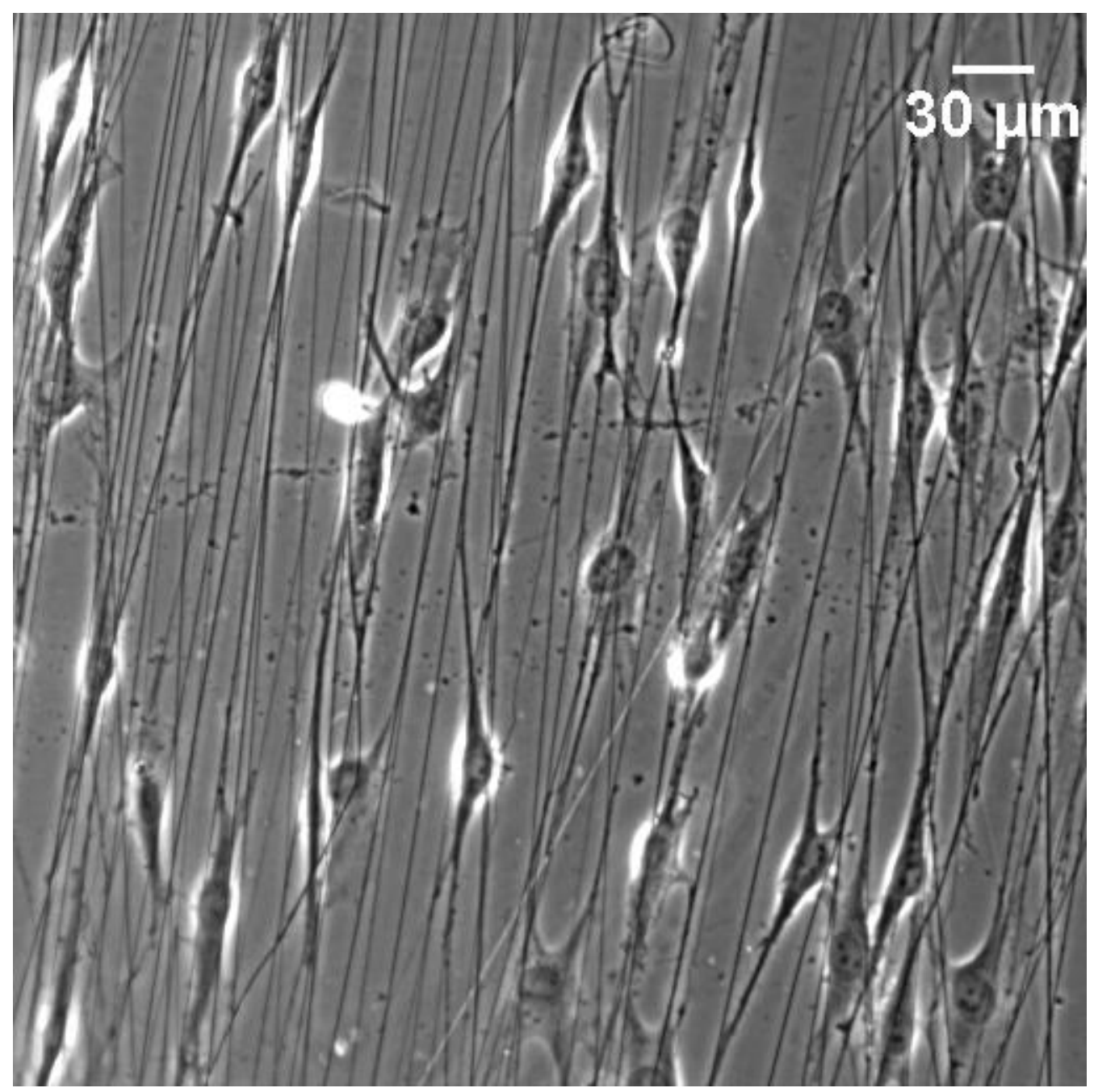

Figure 4.8: 20X magnification of Schwann cells on large fibers. Note the alignment of the nuclei in these cells. The nuclei have an angular deviation of $14.4^{\circ}$.

The first indicator for the formation of bands of Büngner is the alignment of cell nuclei. Nuclei alignment is visible in Figure 4.8. Note the large cell area in Figure 4.6. This spreading is also indicative of the formation of bands of Büngner [9394]. Note the similarities in Schwann cells between Figures 4.9 and 4.6, Schwann cells on electrostatically aligned large nanofibers. Schwann cells in both cases show arrangement into cords, significant cell spreading, nuclei 
alignment, and definitive directionality. Other groups have been able to show bands of Büngner, although not with significant cell spreading [93].

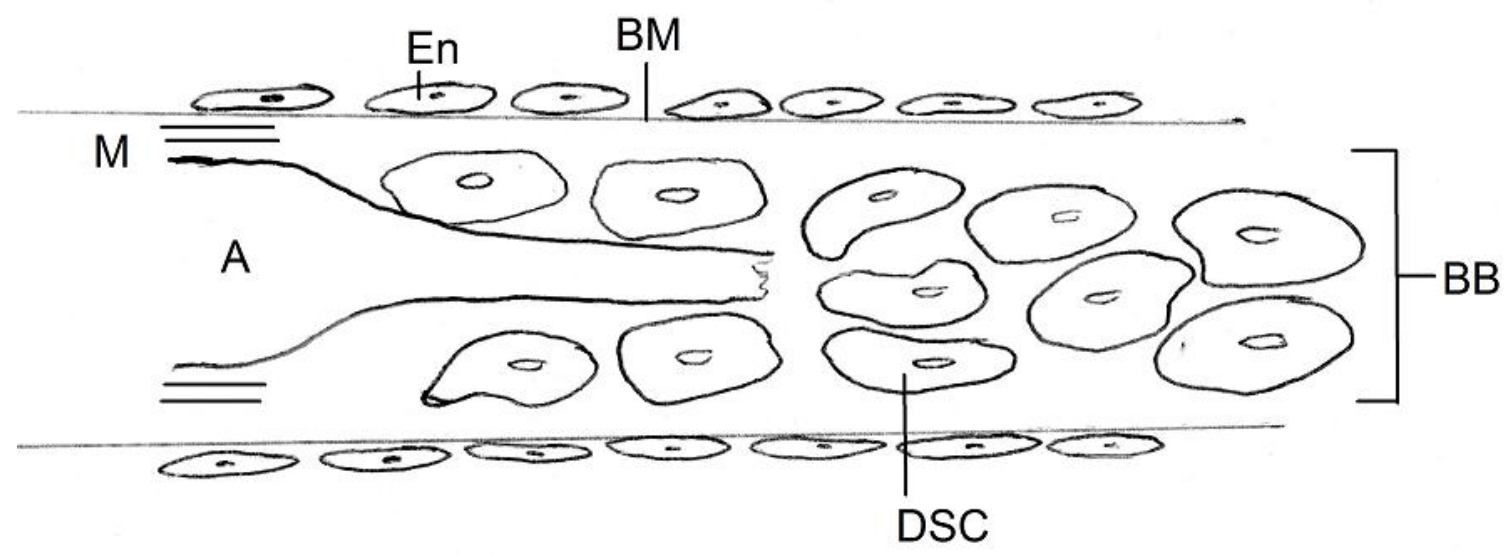

\begin{tabular}{|l|l|}
\hline A & axon \\
\hline BB & Bands of Büngner \\
\hline DSC & Dedifferentiated Schwann cell \\
\hline En & endoneurium \\
\hline BM & Basement membrane \\
\hline M & Myelin \\
\hline
\end{tabular}

Figure 4.9: Bands of Büngner [94]. 


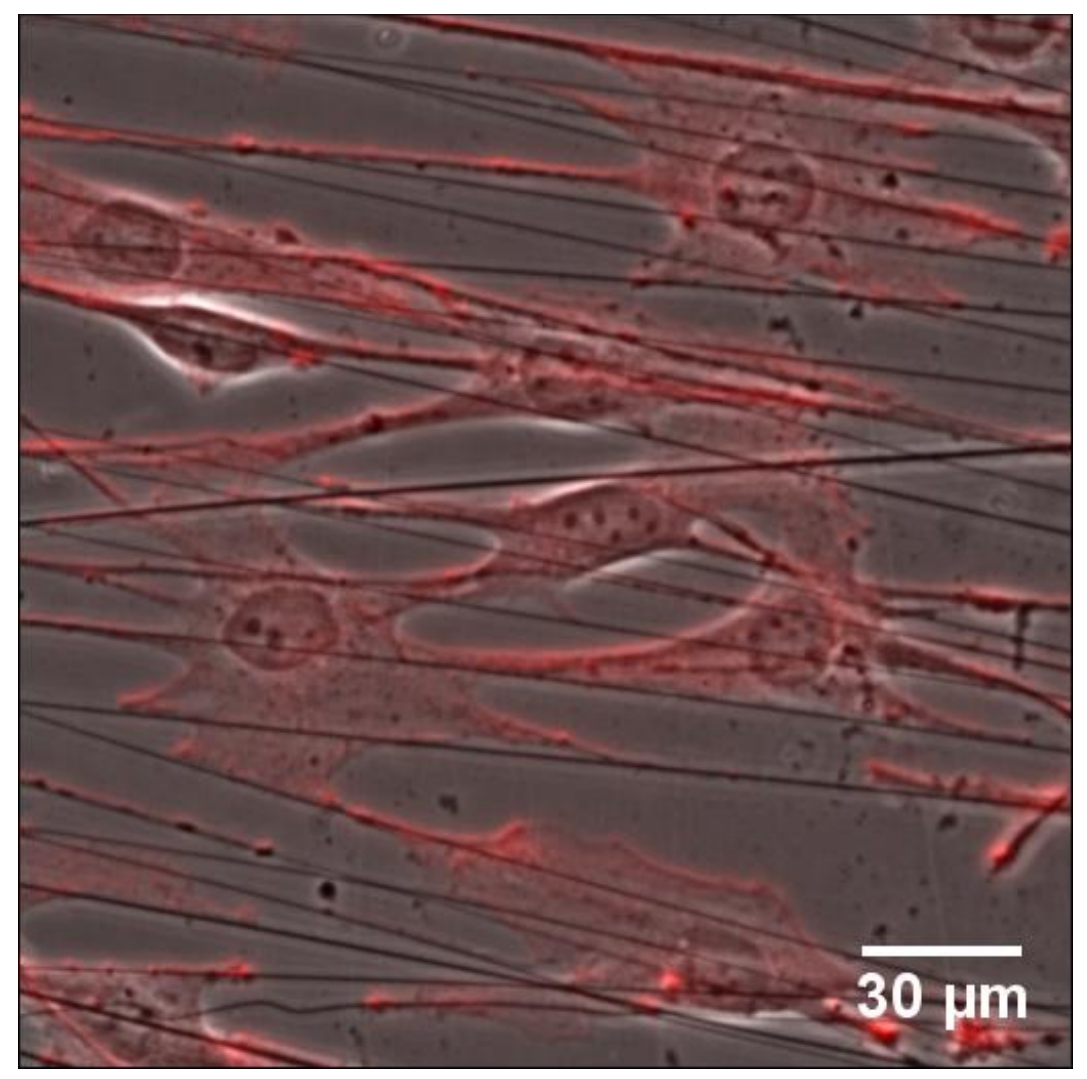

Figure 4.10: 40X magnification image of Schwann cells on large plate nanofibers. Note the large cell area indicated by the cells spreading between several fibers.

Higher magnification images, such as Figure 4.10 show that the cells are spread amongst many adhesion sites in the larger plate nanofibers. Schwann cell seeding on these samples was repeated to verify reproducibility and with different nanofiber mesh densities. All large nanofiber plate samples resulted in the same significant cell spreading.

There is also a significant decrease in length in the cells on the smallest nanofiber samples. This finding provides further evidence that Schwann cells do not adhere well to the smallest nanofibers. Initial evidence for this finding is provided in Figure 4.3, where the length and elongation of the cells is greatly 
subdued. Figure $4.5-4.8$ show that the morphological differences in Schwann cells depend upon fiber alignment methodology. Cells on other samples elongated, aligned, and had aligned nuclei, but did not have as large an area or similar cell morphology as the cells on the large plate nanofibers. These findings suggest that plate aligned large nanofibers could be used as a successful platform for peripheral nerve regeneration in humans.

\subsection{PC12 cell response to highly aligned sets of PLGA nanofibers}

Currently, researchers have used PC-12, or pheochromacytomia-12 cells, an immortalized cell line derived from rat adrenal gland tumors for a good model of primary neurite extension. These cells express neurite-like processes when exposed to Neural Growth Factor (NGF). Research has shown a size dependence to the amount of NGF required to trigger neurite formation [95]. One group showed that nanoscale topographic cues less than $1 \mu \mathrm{m}$ in size triggered neurite expression when exposed to sub-optimal levels of NGF, with the best size for triggering neurite outgrowth between $70 \mathrm{~nm}$ and $400 \mathrm{~nm}$ [95]. This study was performed with perfectly aligned, non-implantable substrates [95]. Others have researched the PC-12 cell adhesion on blended laminin with PCL, noting that neurites formed regardless of the presence of NGF [8]. Additionally the effect of aligned PCL nanofibers between $400 \mathrm{~nm}$ and $1.3 \mu \mathrm{m}$ and respective neurite outgrowths has been explored, but none has examined the optimal diameter for 
maximal PC12 neurite length with highly aligned nanofibers less than 400nm $[8,96-97]$.

\subsubsection{PC12 cell Materials and Methods}

PC12 cell neurite extension was also examined on each of the three fiber diameter ranges. PC12 cells requires poly-D,L-lysine coating for good adhesion to the nanofibers in cell culture, therefore molecular orientation experiments could not be performed. The fibers are coated with polylysine immediately before seeding [98]. Again, we will use the null hypothesis: PC12 cells will have identical alignment and process length across the diameter range. For SEM images, cells were fixed with glutaraldehyde, dehydrated, coated with gold/palladium and imaged. PC12 seeding and cell maintenance procedures can be found in Appendix E. 


\subsubsection{PC12 cell Results and Discussion}

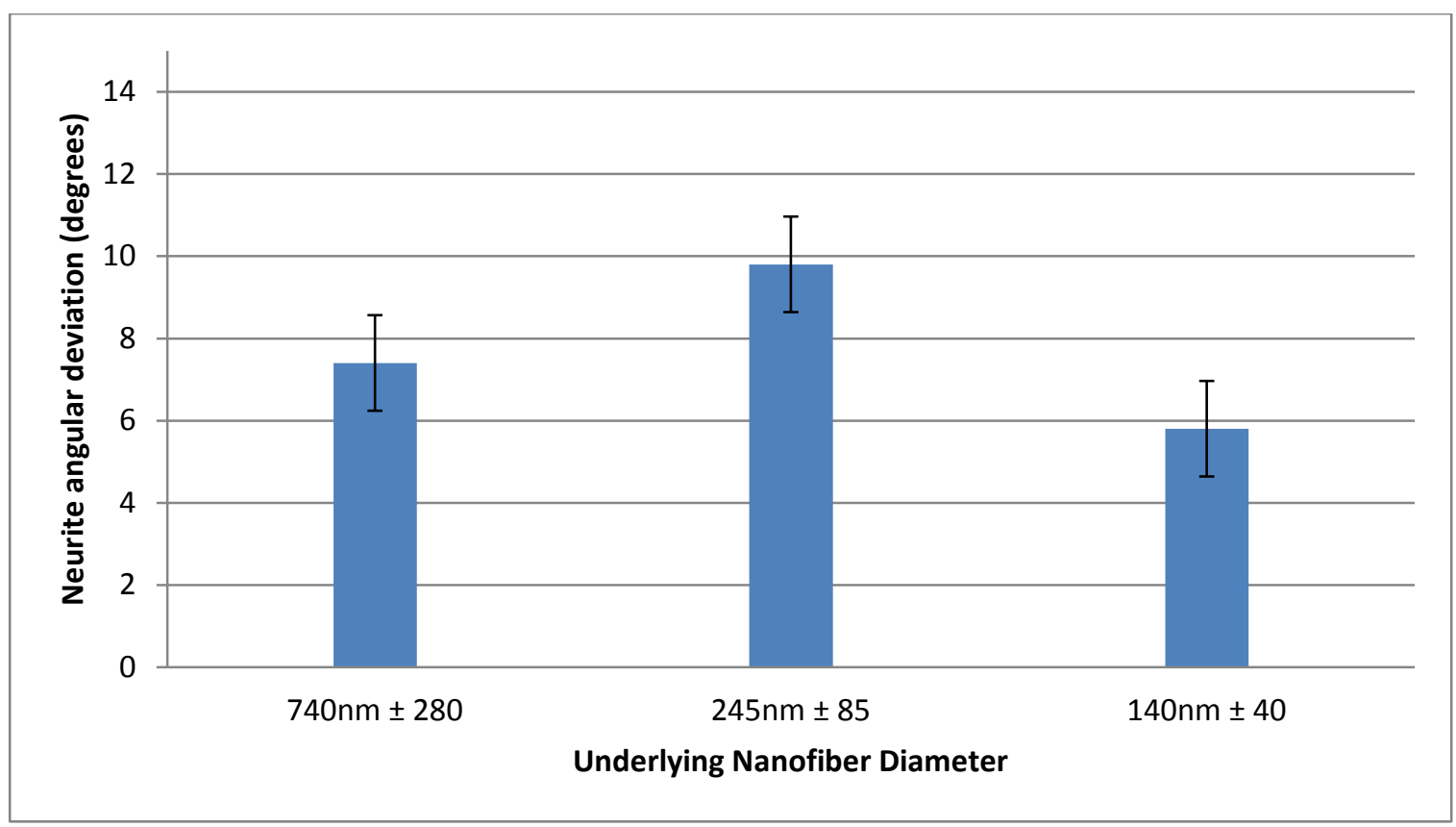

Figure 4.11: PC12 angular deviation. Note how angular deviation is poorer for smaller nanofibers. Angular deviation of underlying nanofibers is $<12^{\circ}$. 


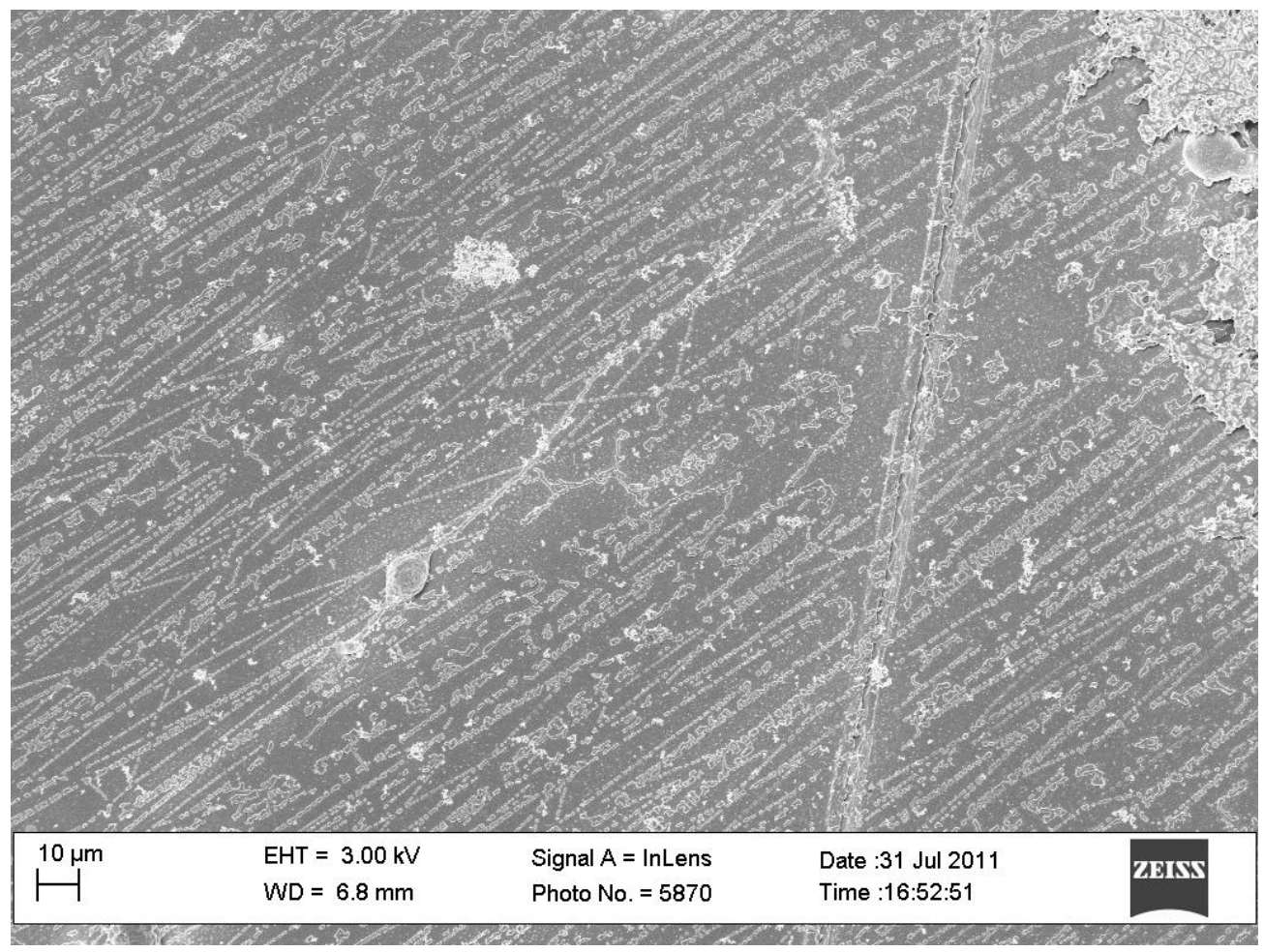

Figure 4.12: PC12 cells on aligned 245nm nanofibers.

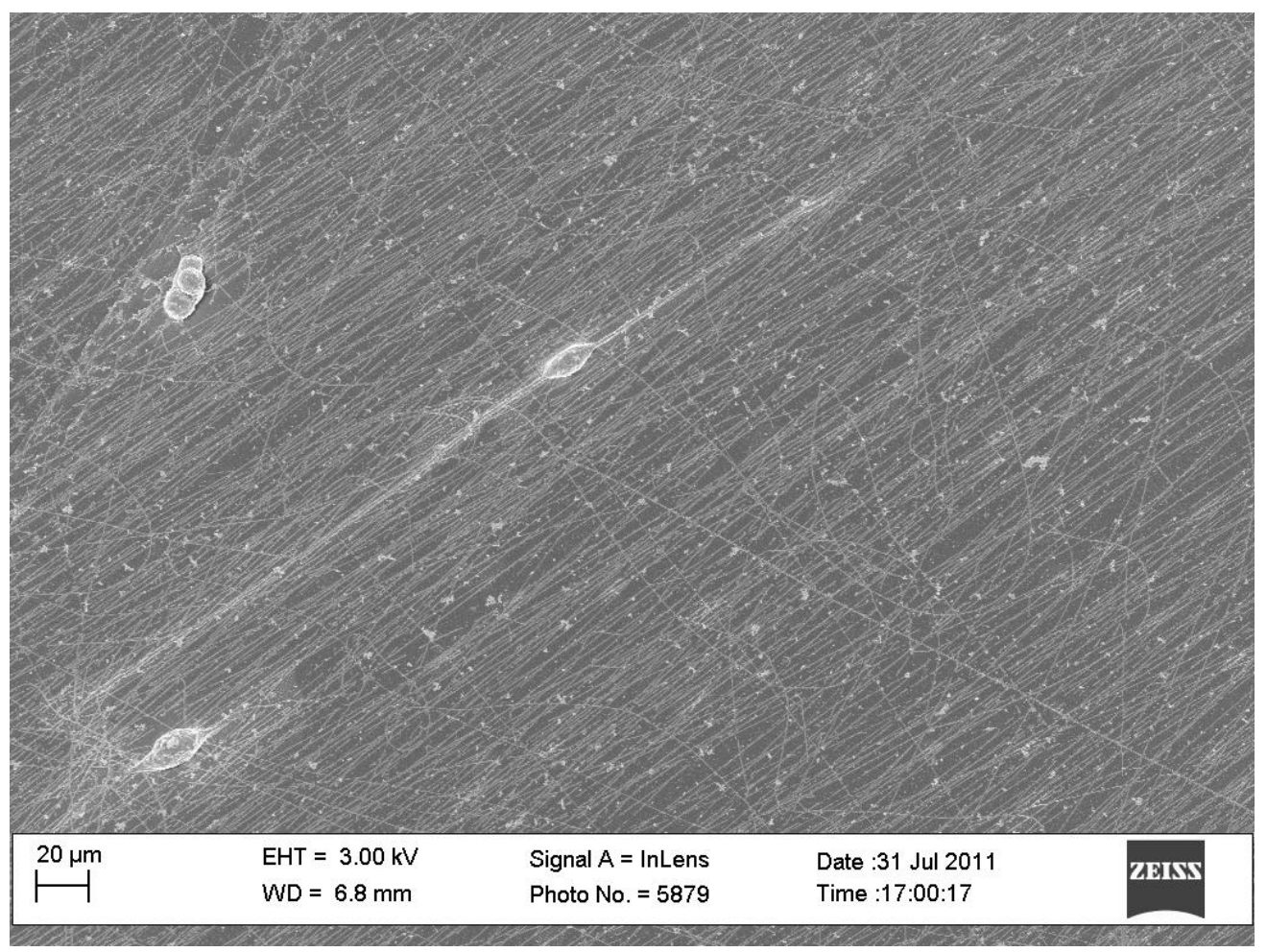

Figure 4.13: PC12 cells on 140nm nanofibers. Note the process elongation. 
These results show that PC-12 cells also preferred the large $(740 \mathrm{~nm})$ and medium $(285 \mathrm{~nm})$ nanofibers over the small $(140 \mathrm{~nm})$ fibers. Cells exhibited poorest alignment on the small (140nm) nanofiber samples, with an angular deviation of $19.9^{\circ}$. The neurite lengths in each case were statistically the same, $70 \mu \mathrm{m} \pm 30$. Percent neurite formation was $29 \%$ for medium and large fibers, but only $20 \%$ for small fibers. This finding is surprising as the literature points to PC12 cells sprouting additional neurites and aligning favorably when presented with smaller nanotopographic cues [95]. PC12 cells had a slightly higher density and neurite density on the medium nanofibers, although this finding was not statistically significant.

\subsection{Conclusions}

In conclusion, Schwann cells exhibited greatest cell spreading on the large plate grown nanofibers. The presumed reason is that the molecular level orientation present in all plate aligned nanofibers. Schwann cells had greater area on the larger $(740 \mathrm{~nm})$ nanofibers versus the smaller (140nm) fibers. PC12 cells also exhibited better alignment on the larger and medium sized nanofibers. Therefore, we expect that nanofiber scaffolds between $285 \mathrm{~nm}$ and $740 \mathrm{~nm}$ in diameter would make the best nervous tissue regeneration conduits, because both the Schwann and PC12 cell response is ideal within this fiber range. This set of experiments advances state of the art because ours is the first group to report Schwann and PC12 cell behavior on small highly aligned nanofibers. We are also the first to 
achieve Schwann cell behavior, in particular, band of Büngner formation on molecularly oriented nanofibers.

\subsection{Summary of Chapter 4}

In this chapter, we seeded Schwann and PC-12 cells on different sized nanofiber samples. In the case of Schwann cells, these fiber samples were grown with different alignment techniques. Schwann cells had larger area on the larger (740nm) plate aligned nanofibers because of the molecular level orientation and additional adhesion sites associated with fibers aligned with plate collectors. The Schwann cells exhibited behavior indicative of PNS injury repair: formation of bands of Büngner. Fibers provided guidance for PC12 cell neurite outgrowth. PC12 cells preferred larger and medium-sized nanofibers. This is the first study to compare highly aligned PLGA nanofibers of sizes from small (140nm) to large (740nm) to determine optimal nanofiber diameter. This study confirmed the work

of $[9,90], \quad 700 \mathrm{~nm}$ nanofibers are better for neural tissue engineering applications. Large plate aligned nanofibers show great promise in neural tissue regeneration because of the better Schwann cell spreading. 


\section{Chapter 5: Coaxial fibers}

\subsection{Introduction to coaxial fibers}

In our previous chapters, we discussed the sensitivity of Schwann and PC12 cells to nanotopographic cues. When exposed to aligned nanofibers, the cells elongated. Another way to produce morphological changes in cells is by using growth factors [19]. Existing scaffolds have examined the release of a single drug, however sequential drug release has not been studied thoroughly [1719,99]. Sequentially released drugs could be very beneficial for regenerating tissues which require more than one signaling factor to trigger growth in the tissue. For instance, first Vascular endothelial growth factor (VEGF), a drug which promotes formation of blood vessels, could be released followed later by $\mathrm{S} 1 \mathrm{P}$, a lipid mediator which promotes the maturation of newly formed blood vessels [100-101].

A coaxial nanofiber is a nanofiber typically composed of two different materials, one on the outside of the nanofiber, referred to as the "sheath" and the other on the inside of the fiber, called the "core." These nanofibers are unusual because they can have different mechanical, physical, and degradation properties in the core versus the sheath. These nanofibers can also provide an unusual drug delivery system in which a drug is in the sheath and a different drug is in the core. The sheath drug will be eluted first as the sheath degrades. Later, the core drug will be eluted from the core after the sheath of the nanofiber has degraded. 
For this research, we will focus on PLGA nanofibers. PLGA nanofibers degrade by hydrolysis which breaks down the polymer chain into their lactic and glycolic acid components. The body then clears these acids. The rate at which they degrade is dependent upon the local $\mathrm{pH}$ (lower $\mathrm{pH}$ will cause the fibers to degrade faster) and how fast water can enter the polymer chain [99]. PLGA with more lactic acid degrades more slowly, because of the hydrophobic methyl group. This phenomenon is discussed in further detail in Chapter 2.

A localized, sequential release could be very useful in tissue regeneration applications, as a sustained release beyond the initial release of a single drug is already very useful for many drug delivery applications [102-103]. The major advantage of using nanofibers over drug eluting nanobeads is that the nanofibers also provide structure and guidance to the tissue.

\subsubsection{Coaxial electrospinning}

To electrospin a polymer coaxially, all one needs in addition to the normal electrospinning setup, is a coaxial needle apparatus. As in standard electrospinning, a high voltage supply drives the collection of polymer onto a grounded collector. In the case of coaxial electrospinning, an additional core solution is pumped into the sheath solution though the use of a coaxial needle. Two different types of polymers can be used, one in the core and one in the

sheath. When selecting core and sheath material, the solvents used in each must be immiscible, and the sheath must be highly electrospinnable [102]. The sheath 
viscosity must be higher than the core, to promote good core formation $[102,104]$.

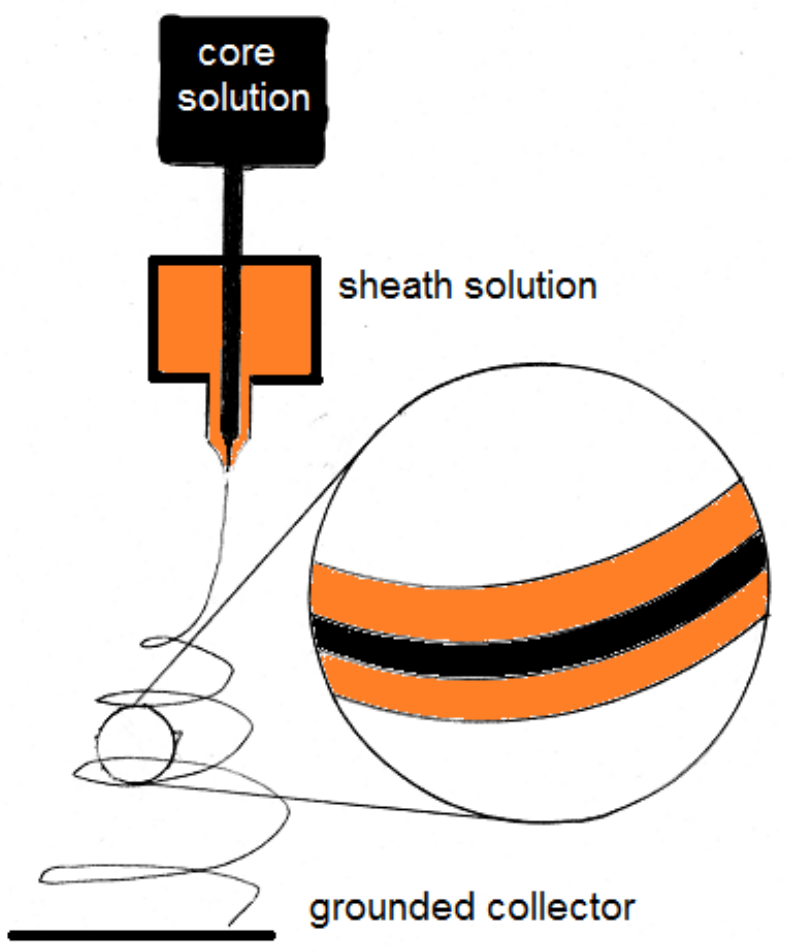

Figure 5.1: Coaxial electrospinning needle and nanofibers.

Coaxial nanofibers are collected on the grounded collector. These fibers can then be used for cell studies, or their drug delivery properties can be examined as they degrade. The voltage and working distance used must also be optimized for the coaxial electrospinning process. Too low a voltage prevents good nanofiber formation; too high a voltage results in separation of the core and sheath materials [102,104]. 


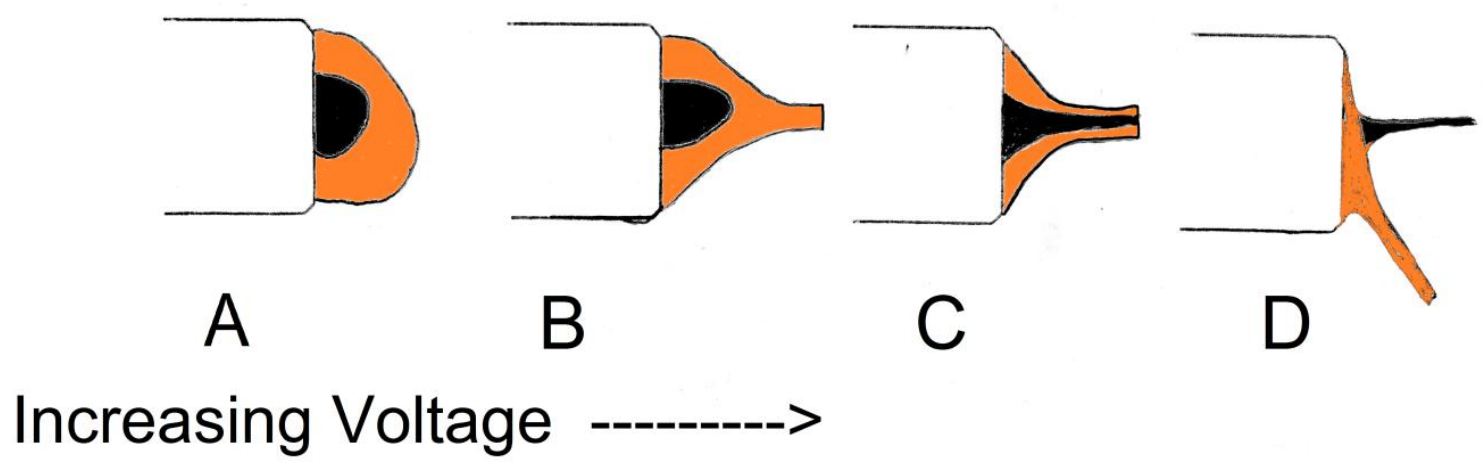

Figure 5.2: Taylor cone formation in coaxial nanofibers with increasing voltage. Note that sub-threshold voltage, as seen in A, prevents good core formation. At $B$, the sub-threshold voltage has been reached and a non-coaxial nanofiber is formed. At $\mathrm{C}$, the voltage is ideal for forming coaxial nanofibers. At $\mathrm{D}$ the voltage is too high and the core and sheath are spinning separately [102].

\subsubsection{Characterizing coaxial nanofibers}

There are many techniques for verifying that the electrospun nanofibers are coaxial. These include freeze-fracture SEM, TEM, and fluorescence microscopy. Freeze-fracture SEM freezes the nanofibers in liquid nitrogen. The fibers are then fractured with a scalpel and an SEM is taken along the fracture, that is, a crosssection of the fiber [102,105-106]. Transmission Electron Microscopy (TEM) can be performed if the two polymers have different enough molecular conductivity properties [28]. Fluorescent dyes can also be used to verify that the nanofibers are coaxial, by dissolving one dye in the core and another in the sheath, or dissolving a dye in the sheath [104-105,107]. Two common dyes that one could use are called Dil and DiO. These dyes fluoresce in the orange and green spectra respectively. One can also verify that the nanofibers are coaxial by a drug elution study [99,108-109], in which materials enveloped in the fibers are 
slowly released as the sheaths of the fibers degrade. Most fibers as reported in the literature are proved to be coaxial nanofibers by freeze fracture SEM. Fibers examined in the literature using fluorescence microscopy never appear truly coaxial. They only appear to have fluorescent material discontinuously incorporated in the nanofiber mesh, as seen in Figure 5.3.
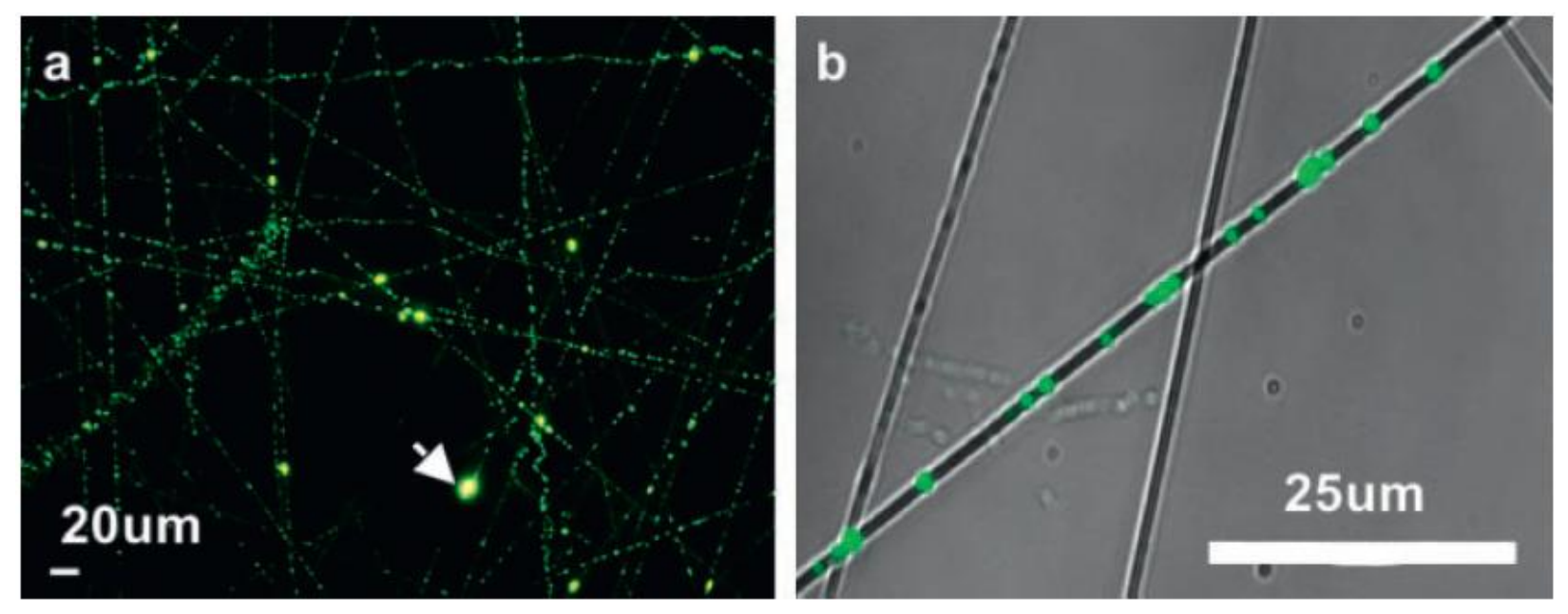

Figure 5.3: Fluorescent particles in PCL core/sheath coaxial fibers. Note the discontinuous nature of the nanoparticles in these fibers. This is from improper mixing of the dye in the polymer solution. Note the fluorescent bead indicated by the arrow in image a. Adapted from [99].

\subsubsection{Drug delivery with coaxial nanofibers}

A nanofiber scaffold that provides both mechanical signals for cellular growth and chemical signals to modify cell behavior would be very useful in tissue engineering. Current research has examined coaxial nanofibers eluting a single drug $[18,106,111]$, as well as two drugs [99]. Coaxial nanofibers could provide a unique drug delivery system by locally delivering two different drugs sequentially, 
without the need for injections or second site implantation surgery $[99,106,109]$. Polymers used in these previous studies are PCL sheath with gelatin or chitosan in the core or PCL and PEG $[28,99,104-105]$. Most groups synthesize coaxial nanofibers examine two different polymers, one in the core and one in the sheath $[28,105,109]$, while few research groups synthesize core/sheath fibers from the same polymer [112].

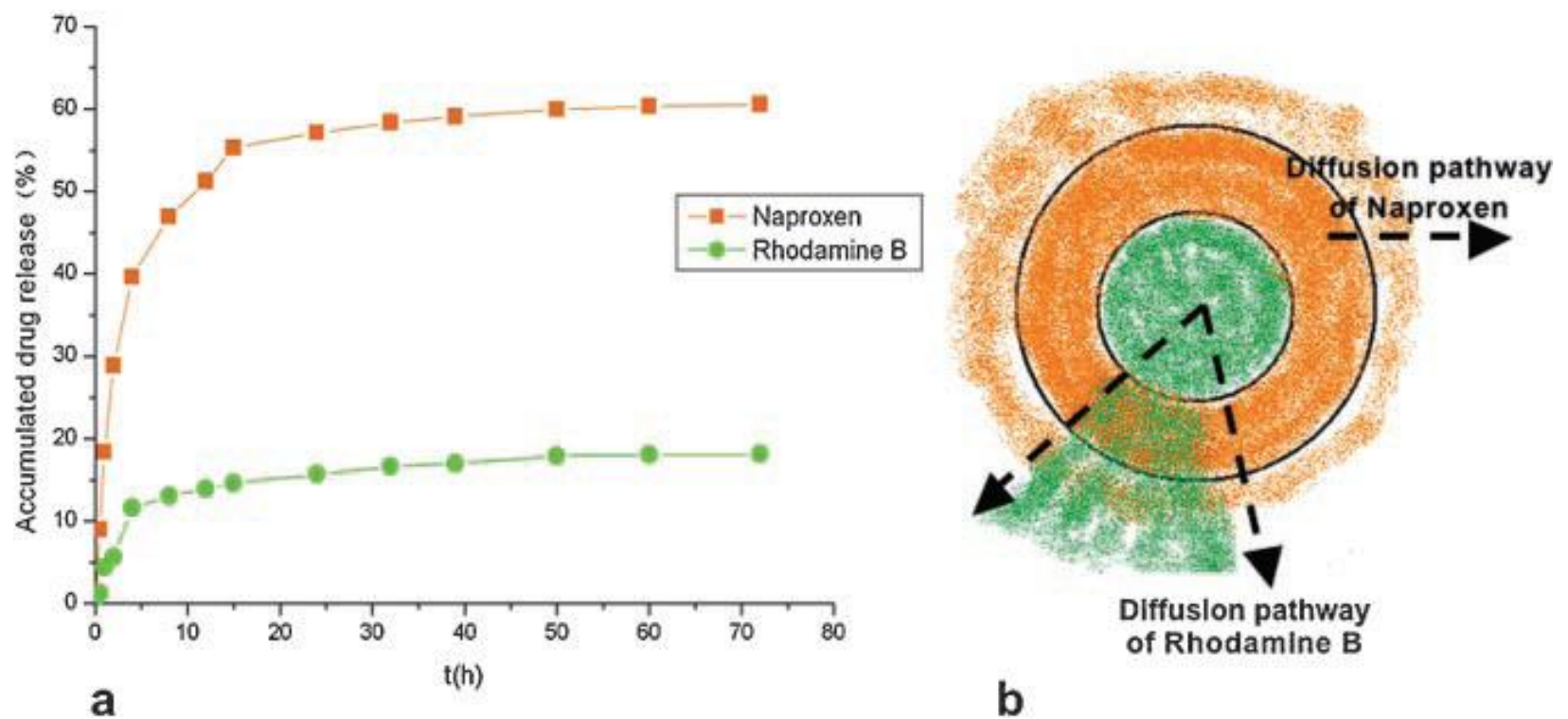

Figure 5.4: Coaxial release profile of two drugs released simultaneously. Adapted from [99].

In our proposed application, the coaxial nanofibers would need to degrade over time as they elute each drug. A common way to interpret results from a drug elution study is by high-performance liquid chromatography (HPLC), in which the nanofibers degrade in aqueous media and samples of the media are taken periodically and analyzed. One could also tag one or both drugs with radioactive atoms. An alternative approach would be to fluorescently tag each drug with a 
different fluorescent dye, and observe the amount of drug present in the supernatant released from the fibers every few hours.

The goals of this research are to create a coaxial fiber platform technology using PLGA core and sheaths for dual drug delivery. First, we will verify formation of coaxial fibers using fluorescent microscopy. This approach has not been convincingly presented in the literature. Fibers of a few microns in size are required, as one cannot resolve nanoscale objects using optical microscopy. Next, we will observe the degradation kinetics of these fibers with respect to a non-coaxial fiber made from 85:15 and 50:50 blended PLGA. Finally, we will deliver two drugs to verify the presence of drugs in both the core and sheath. This work will advance the state of the art because no group has published PLGA core/sheath coaxial dual drug eluting fibers. PLGA is a good candidate for drug delivery applications because of its biocompatibility and selectable biodegradation properties. This research provides a stepping stone for same material core/sheath coaxial electrospinning and future research in dual drug delivery.

\subsection{Materials and Methods}

For the first set of fibers, PLGA 50:50 and 85:15 were dissolved in pure THF in the sheath and DCM in the core. The dyes Dil and DiO were used to fluorescently label the core or sheath. Different solvents were used to prevent mixing of the solutions during the coaxial electrospinning process. These 
solvents, when loaded with polymer, are immiscible. Fibers were electrospun using a 18 and 25 gauge coaxial needle setup.

\begin{tabular}{|l|l|l|l|l|l|}
\hline Location & Polymer & $\%$ Polymer & Solvent & Dye & Flow rate \\
\hline Core & PLGA 85:15 & $40 \%$ & DCM & & $0.1 \mathrm{~mL} / \mathrm{hr}$ \\
\hline Sheath & PLGA 50:50 & $20 \%$ & THF & Dil & $1 \mathrm{~mL} / \mathrm{hr}$
\end{tabular}

Figure 5.5: PLGA 85:15 coaxial fiber electrospinning conditions for the coaxial fiber.

\begin{tabular}{|l|l|l|l|l|l|}
\hline Location & Polymer & $\%$ Polymer & Solvent & Dye & Flow rate \\
\hline Core & PLGA 50:50 & $40 \%$ & DCM & Dil & $0.1 \mathrm{~mL} / \mathrm{hr}$ \\
\hline Sheath & PLGA 50:50 & $20 \%$ & THF & DiO & $1 \mathrm{~mL} / \mathrm{hr}$ \\
\hline
\end{tabular}

Figure 5.6: PLGA 50:50 nanofiber electrospinning conditions for the coaxial nanofiber.

Care was taken to ensure proper Taylor cone formation by frequent cleaning of the coaxial needle and careful monitoring of the voltage. Fibers were collected on glass slides for observation under fluorescent microscopy.

For the third set, fibers were synthesized for an elution study. These fibers were coaxially electrospun with a PLGA 50:50 sheath and Inulin-FITC in PLGA 85:15 or PLGA 50:50 as a fluorescent additive in the core using the conditions listed in Figures 5.5 and 5.6. Inulin-FITC was chosen because it is very inexpensive, and typically several $\mathrm{mL}$ of core solution are required to create coaxial nanofibers. Elution studies were performed on these fibers by placing identical weights of fibers into dark vials. Deionized (DI) water was added to each vial. The vials were 
kept at 37 degrees $C$ and agitated over the course of the 100 hour study. Supernatant was removed every few hours, with fresh DI added to replace the amount removed. Care was taken to ensure that the samples were not exposed to excess light. Change in fluorescence from the supernatant was observed using a NanoDrop 100 fluorescent reader (Thermo Scientific, Wilmington, DE).

For the last set of fibers, PLGA 85:15 core PLGA 50:50 sheath nanofibers were loaded with fluorescently labeled heparin in the core and fluorescently labeled BSA in the sheath.

\begin{tabular}{|l|l|l|l|l|l|}
\hline Location & Polymer & $\%$ Polymer & Solvent & $\begin{array}{l}\text { Fluorescent } \\
\text { material }\end{array}$ & Flow rate \\
\hline Core & PLGA 85:15 & $40 \%$ & DCM & Heparin-AF647 & $0.1 \mathrm{~mL} / \mathrm{hr}$ \\
\hline Sheath & PLGA 50:50 & $20 \%$ & THF & BSA-FITC & $1 \mathrm{~mL} / \mathrm{hr}$
\end{tabular}

Figure 5.7: coaxial fiber electrospinning conditions for the dual drug eluting coaxial fiber.

A degradation study was performed on these fibers as described previously with a 72 hour timeframe to observe the release of these two materials.

\subsection{Results}

Fluorescent images of coaxial fibers are depicted below. 


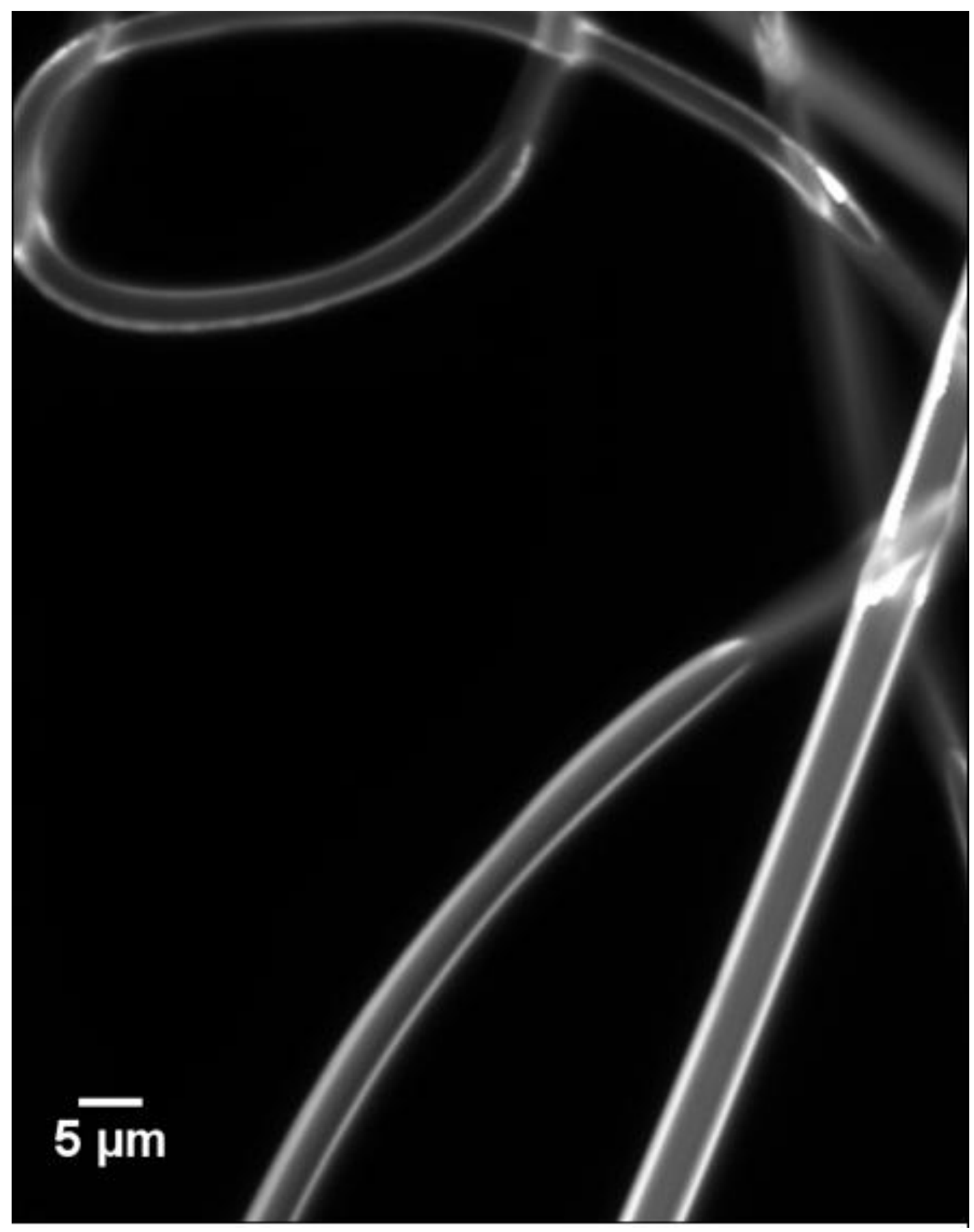

Figure 5.8: PLGA 85:15 core and PLGA 50:50 sheath coaxial fibers. White color is the fluorescent dye. Note that only the sheath is labeled with fluorescent dye. Therefore these fibers appear to have a hollow core. 


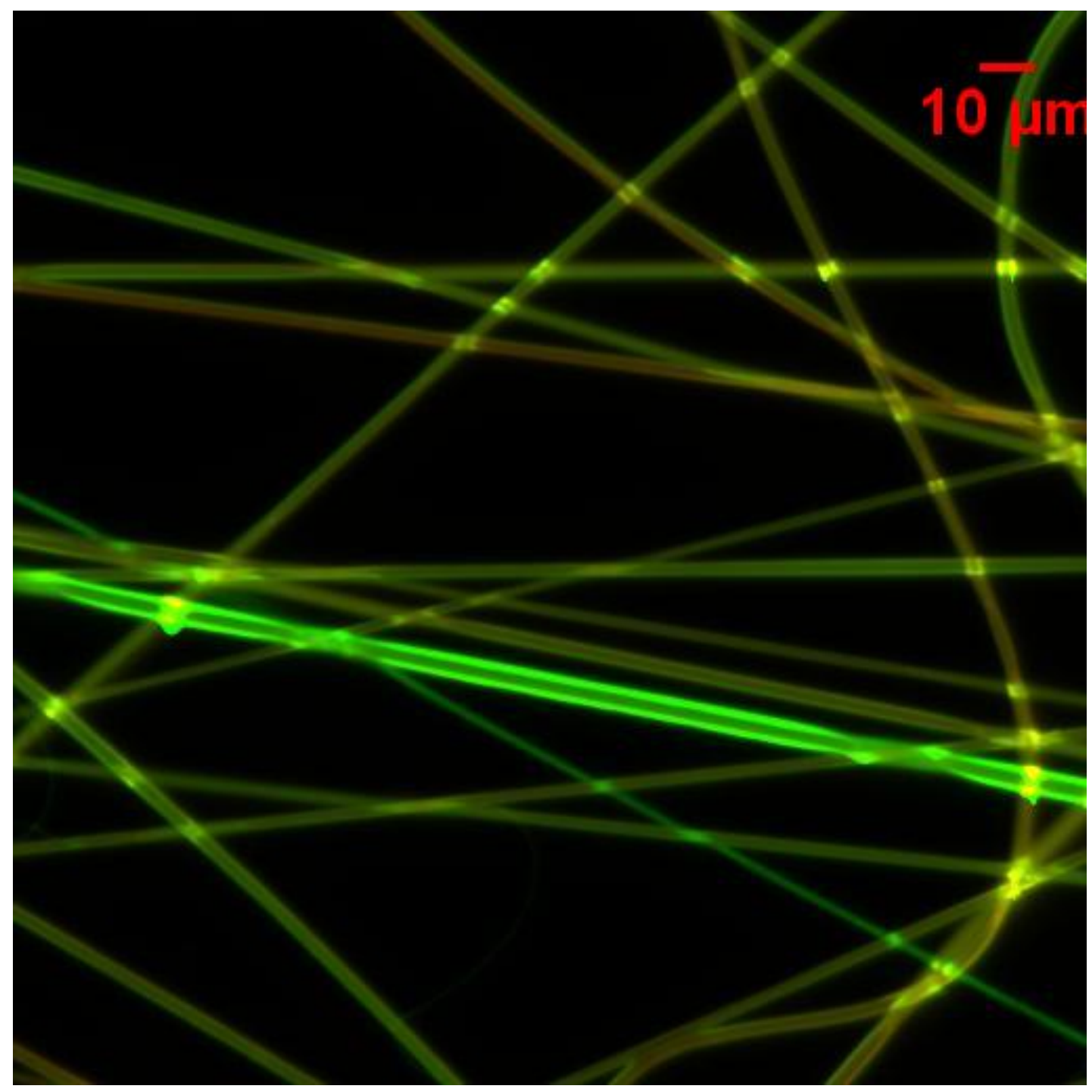

Figure 5.9: PLGA 50:50 coaxial core and sheath fibers. Note the red core and green sheath visible in larger micron-sized fibers. The smallest fibers in this image are nanofibers, approximately $700 \mathrm{~nm}$ in diameter. 

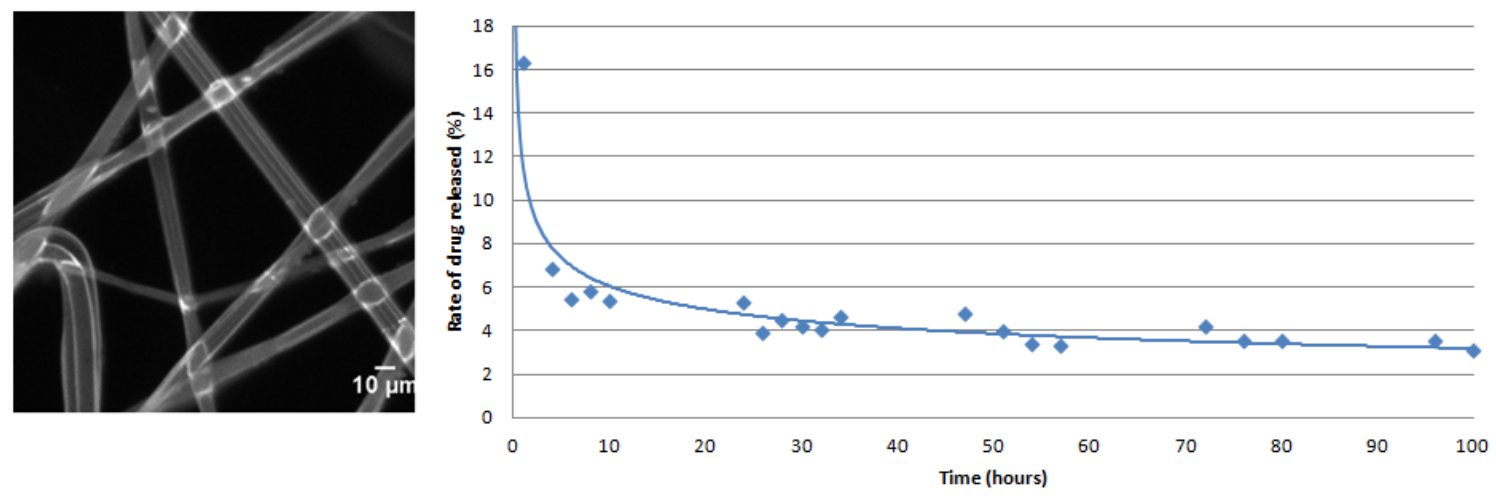

Figure 5.10: PLGA 85:15 Inulin-FITC core fibers and release profile. Y-axis in graph is the rate of release of total percent of drug released. Note how after the initial burst release a sustained release is achieved.
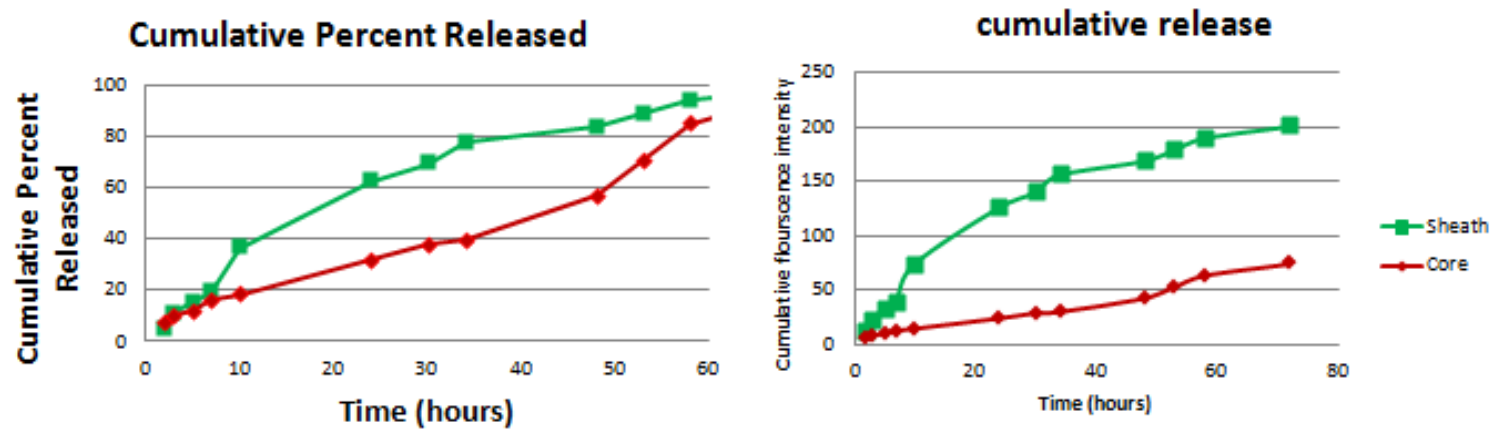

Figure 5.11: Release profile from PLGA 85:15 with AF-647 labeled heparin in the core and PLGA 50:50 with FITC labeled BSA in the sheath. 


\subsection{Discussion}

From these results, we can see that we have optimized the electrospinning parameters to yield coaxial fibers a few microns in diameter. PLGA 85:15 was successfully encapsulated in the core. PLGA 50:50 core and sheath microfibers were also synthesized, as seen in Figure 5.8. Several fibers were on the nanoscale.

PLGA 85:15 core fibers performed very well with respect to the control when releasing inulin-FITC. PGLA 50:50 fibers released inulin-FITC in an uncontrolled manner, and therefore were not selected for future experiments. PLGA 85:15 core fibers exhibited a very controlled, sustained release as evident by the smoothness in the curve in Figure 5.10. The blended fibers released the inulinFITC via diffusion dominated release kinetics, but the release profile from the 85:15 core fibers was uniform after the initial burst release.

Because of this highly controlled release profile in Figure 5.10, a dual release study was performed on PLGA 85:15 core 50:50 sheath fibers. These fibers released the drug in the sheath first and had a sustained release of the heparin-

alexafluro 647 after 48 hours, as evidenced in Figure 5.11. The maximum release from the sheath occurred within a 30 hour timeframe. The maximum rate of molecular release for the sheath occurred at 24 hours, while the maximum rate of molecular release from the core occurred at 48 hours. This proves that the core had a sustained release dampened by the sheath. The both the core and sheath followed Higuchi release kinetics with $r$ values of 0.9882 and 0.9953 
respectively. Higuchi release kinetics indicate a diffusion controlled reaction. The reason for the slightly delayed release of heparin is it must first diffuse from the core through the sheath before its release.

These fibers can be used as a platform technology for many drug delivery applications. This is the first work to present PLGA core/shell coaxial fibers for drug delivery applications.

\subsection{Conclusion}

In conclusion, we were able to synthesize coaxial fibers with PLGA 85:15 in the core and 50:50 in the sheath. PLGA 50:50 coaxial nanofibers were synthesized, however they did not release inulin-FITC in a controlled manner. Because PLGA $85: 15$ core $50: 50$ sheath fibers performed the best compared to control, a dual drug elution study was performed. PLGA 85:15 coaxial fibers eluted both drugs in a controlled manner, with maximum release of BSA occurring after 24 hours and maximum release of the heparin occurring after 48 hours. The process developed in this research can be used as a platform technology to create dual drug releasing materials utilizing our $85: 15$ core $50: 50$ sheath coaxial fibers. 


\subsection{Summary of Chapter 5}

We were able to successfully synthesize PLGA 85:15 core with PLGA 50:50 sheath coaxial fibers. This is advancing state of the art as no other research group has reported dual drug delivery from same material core/sheath coaxial fibers. PLGA 85:15 core and PLGA 50:50 sheath fibers had a very controlled release profile. These fibers eluted the encapsulated molecules in a very controlled fashion through diffusion. These fibers were used to verify that one can release two drugs coaxially with a PLGA 85:15 fiber core and 50:50 fiber sheath. We were also able to successfully and convincingly use fluorescent labeling for the sheath and core to verify our coaxial fibers. 


\section{Chapter 6: Conclusions}

In this work, we began by exploring the relationship between nanofiber diameter and alignment. In chapter 2, we explored synthesizing fibers of different diameter. We found that through altering the dielectric constant of the polymer solution we were able to synthesize smaller and smaller nanofibers. We derived an equation (eq. 2.4) which can be used to tailor nanofiber diameter through modification of the polymer's dielectric constant. The PLGA 85:15 nanofibers presented in are smaller and better aligned than any others that have been reported in literature. The deliverable created from this chapter was an equation that relates dielectric strength of the solvent to nanofiber diameter. We found that these nanofibers of smaller size required smaller gap widths to align well, as following our group's model. We also explored dielectric electrospinning, and discovered challenges associated with aligning fibers of smaller size on dielectric block collectors, as well as solutions to many of these problems.

In chapter 3, alignment of differently charged polymers was examined. We found that for electrostatic techniques, polymers of higher charge do not align as well as lesser-charged polymers. We found that polymers of medium charge followed our developed model well, stating and thus they required smaller gap widths to align well, and had alignment directly dependent upon gap width. Large nanofibers align well with mechanical techniques regardless of their charge. We were the first group to explore how these charges effect nanofiber alignment. 
In chapter 4, Schwann and PC12 cells were seeded on nanofibers synthesized in this research. We determined that Schwann cells exhibited different morphologies based upon fiber alignment technique. Schwann cells had the most spreading on the largest diameter nanofiber samples that were synthesized with plate electrospinning techniques. These Schwann cells resembled the bands of Büngner, an important initial step in peripheral nerve regeneration. PC12 cells exhibited good directionality and elongation on medium and large sized nanofibers. These experiments suggested that large plate aligned nanofibers should be used for future in vivo peripheral nerve regeneration experiments. We advanced state of the art by determining the nanofiber diameter and alignment technique at which the formation of bands of Büngner is triggered. We were the first group to trigger band of Büngner formation on nanofibers.

In chapter 5, coaxial nanofibers composed entirely of PLGA were synthesized. We observed diffusion mediated release kinetics from coaxial nanofibers and were able to develop coaxial PLGA 85:15 core and 50:50 sheath nanofibers as a platform technology for future drug delivery applications. Ours is the first group to achieve coaxial PLGA in PLGA micro and nanofibers and thoroughly document them using fluorescent microscopy. Dual drug releasing fibers show great promise in tissue engineering because one could initially release a drug to improve cell behavior during the early healing phase and a later release a drug to promote cellular maturation. 


\begin{tabular}{|c|c|c|}
\hline Chapter & Previous state of the art & New state of the art \\
\hline 2 & $\begin{array}{l}\text { - Fibers }<200 \mathrm{~nm} \text { aligned with } 30^{\circ} \\
\text { angular deviations without the use of } \\
\text { nanoparticles }[9-10,26,35,39] \\
\text { - No systematic way to make nanofibers } \\
\text { of given diameter }[42,47]\end{array}$ & $\begin{array}{l}\text {-Small }(140 \mathrm{~nm}) \text { PLGA nanofibers aligned } \\
\text { with AD's }<15^{\circ} \\
\text {-Developed formula which allows } \\
\text { modulation of fiber diameter by } \\
\text { dielectric constant } \\
\text { - Well documented methodology for } \\
\text { aligning nanofibers of a given diameter }\end{array}$ \\
\hline 3 & $\begin{array}{l}\text {-No research on polymer inherent } \\
\text { charge [40] }\end{array}$ & $\begin{array}{l}\text {-Studied the effects of internal and } \\
\text { residual charge on nanofiber alignment }\end{array}$ \\
\hline 4 & $\begin{array}{l}\text { - } \mathrm{NIH}-3 \mathrm{~T} 3 \text { cell response evaluated on } 30^{\circ} \\
\text { angular deviation nanofibers of smaller } \\
\text { size [9-10] } \\
\text { - Band of Büngner formation with small } \\
\text { cell area triggered on polylysine coated } \\
22 \mu \mathrm{m} \text { PCL microgrooves [93] } \\
\cdot \mathrm{PC} 12 \text { neurite formation evaluated on } \\
1.3 \mu \mathrm{m} \text { and } 400 \mathrm{~nm} \mathrm{PCL} \text { nanofibers [96- } \\
97]\end{array}$ & $\begin{array}{l}\text {-Schwann cell response evaluated on } \\
\text { small, highly aligned nanofibers } \\
\text {-Band of Büngner formation with large } \\
\text { cell area triggered on molecularly } \\
\text { aligned uncoated PLGA 740nm } \\
\text { nanofibers } \\
\text { •PC12 neurite formation evaluated on } \\
\text { highly aligned (700-140nm) PLGA } \\
\text { nanofibers }\end{array}$ \\
\hline 5 & $\begin{array}{l}\text {-Coaxial micro and nanofibers formed } \\
\text { with PCL in core and/or sheath }[12,107] \\
\text {-Chitosan/PCL core/sheath dual drug } \\
\text { eluting fiber [99] } \\
\text {-Inconclusive fluorescent sheath and } \\
\text { core }[99,107-109]\end{array}$ & $\begin{array}{l}\text { - Coaxial micro and nanofibers formed } \\
\text { with PLGA in core and sheath } \\
\text {-PLGA/PLGA core/sheath dual drug } \\
\text { eluting fibers } \\
\text { - Conclusive fluorescent sheath and core }\end{array}$ \\
\hline
\end{tabular}

Figure 6.1: Advances in the state of the art. 


\section{Chapter 7: Future work}

Technology developed from this research can be taken in a number of directions. This section on future work aims to directly expand work in this document.

As described in Chapter 2, electrospinning can be used to synthesize nanofibers for tissue engineering. The important factors to consider for these nanofibers are their diameter and angular deviation. Future work to improve both attributes is needed, although as our work has shown, smaller is not always better.

First, one could continue to explore the diameter and solvent dielectric relationship by using different polymers for the electrospinning process, such as poly-methyl methacrylate or additional polyphosphazenes with desirable mechanical characteristics.

Further research must be done in the polymer/solvent interaction model. The current model states that the solvent enters the bulk polymer and provides lubrication between the long chains of the polymer. This solvent action allows the polymer to enter into solution and later be electrospun. This model should be verified in its entirety.

To aid in alignment one could apply reeling technology to electrospinning. One of the main requirements in winding wire, yarn, or fishing line on a spool is an oscillating fiber source. This could be implemented in electrospinning by placing the syringe pump on a moving stage. The speed at which the syringe pump travels could then be altered to achieve ideal fiber coverage and alignment. 
Another thought for improving fiber alignment is utilizing developments in fishing rod technology. Zebco revolutionized the fishing rod industry in the 1950's with the invention of the Zebco fishing reel. The Zebco reel worked differently from any other reels available at that time. Engineers there figured out that if one were able to rotate the reel $90^{\circ}$ from the direction of the line, then the reel would collect the line very much more smoothly [113].

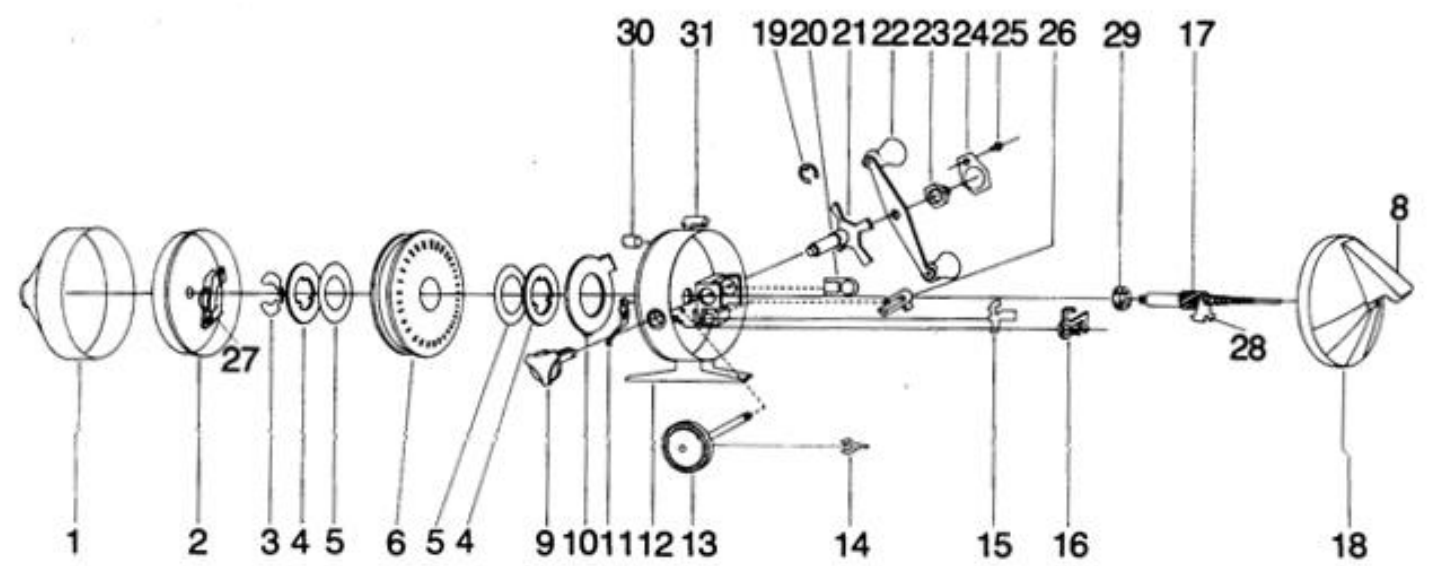

Figure 7.1: Breakdown view of a Zebco 1 classic fishing reel. Line enters the reel from the left through the hole in the front cover (1). The line is then rotated $90^{\circ}$ by the pickup arm (27) before it is wound onto the reel (6). When the button (8) is pressed, the pickup arm (27) moves out of the way, allowing the line to be cast free of impediment. Adapted from [111].

This technology could be adapted to electrospinning by collecting fibers on the mandrel, rotated $90^{\circ}$ from the syringe pump. The fibers could be repelled by a positively charged rod or bar towards the rotating mandrel. Alternatively, circular, positively charged eyelets could be used to "take up the slack" in smaller nanofibers, and approach which should result in better fiber alignment. 
Further testing of the relationship between dielectric constant and nanofiber diameter is required, in particular, apply the formula to other polymers.

Additional polymers with different charge densities can be evaluated to further examine the charge on the polymer backbone with degree of alignment. For instance, EG Methyl, a different polyphosphazene could be investigated for its charge properties. Gap widths on dielectric collectors could also be altered to determine the role that gap width of dielectric collectors plays on highly charged nanofiber alignment.

PC12 / Schwann cell co-cultures could be performed on these plate aligned nanofibers. Primary neuronal studies should also be performed. Additional cells of larger and smaller native cell diameter could be examined on nanofibers of different size. Gene expression studies could be performed on Schwann cells to look for L1 expression, the adhesion molecule associated with the formation of bands of Büngner [93]. PC12 cells could be examined for protein 43 (GAP-43) and synapsin I upregulation, both genetic markers associated with PC12 regeneration. Finally, these plate aligned nanofibers can be used in animal and human models to evaluate any improvement in healing time achieved by the nanofiber scaffold. For instance, rabbit ulnar nerves could be severed and the repair time with and without implanted aligned nanofiber meshes could be evaluated. 
Furthermore, one could expand the successful use of two-dye systems in other micro and nanofibers. Further development of materials which could sequentially deliver two different drugs (drug A for a period of time followed by drug B) would be very desirable. For instance, one could first release Vascular endothelial growth factor to trigger initial angiogenesis, and then later could release S1P to promote maturation of the newly formed blood vessels. 


\section{Acknowledgements}

Prof. Ron Williams

Prof. Nathan Swami

Prof. Ed Botchwey

Prof. Tim Allen

Prof. Shayn Peirce-Cottler

Gerrit Van Gilst

Rachel Su

Vasudha Chaurey

Brian Schmidt

Christoph Wilhelm

Brian Mulchaey

Trevor Wesolowski

Anusuya Das

Frank E. Block, Jr.

Stephanie Chen

Richard White 


\begin{abstract}
Alex Lobo
Mikiyas Tsegaye

Matthew Peach

Damond Cromer
\end{abstract}

Rebekah Neal

Meng Deng

Prof. Lakshmi Nair

Prof. Yusuf Khan

Richard Block 


\section{Publication}

This dissertation has been submitted for publication as follows:

- Chaurey, V., Block, F. Su, Y., Chiang, P., Botchwey, E., Swami, N. Sensitivity of fibroblast cell directionality to surface charge uniformities on aligned electrospun nanofibers. Biomaterials. Submitted for publication, February 2012.

- Block, F. Chaurey, V., Swami, N. Influence of residual charge on alignment of electrospun nanofibers at insulator and dielectric gap collectors. Manuscript in preparation

- Block, F. Van Gilst, G., Swami, N. PLGA core/sheath microfibers for drug delivery applications. Manuscript in preparation 


\section{Bibliography}

1. Clarke, A. C. Profiles of the future. Henry Holt \& Co. 1984.

2. Genesis 2:21 New International Version

3. Atala, A. and Nyberg, L. Tissue engineering. World Journal of Urology. 2000. 18:1.

4. Viola, J. et al. The Emergence of Tissue Engineering as a Research Field. Abt Associates Inc. 2003.

5. Wolter, J. \& Meyer, R. Sessile Macrophages Forming Clear Endothelium-like Membrane on Inside of Successful Keratoprosthesis. Transactions of the American Ophthalmological Society. LXXXII. 1984.

6. MacArthur, B. \& Oreffo, R. Bridging the gap: Tissue Engineering: mathematical models are helping to take tissue engineering from concept to reality. Nature. 2005. 443 (7021), 19.

7. Gabriel, A. et al. Wound Healing, Growth Factors. Medscape Reference. http://emedicine.medscape.com/article/1298196-overview 2009.

8. Neal RA, Tholpady SS, Foley PL, Swami N, Ogle RC, Botchwey EA. 2012. Alignment and composition of laminin-polycaprolactone nanofiber blends enhance peripheral nerve regeneration. J Biomed Mater Res Part $A$ 2012:100A:406-423.

9. Bashur, C., Dahlgren, L. Goldstein, A. Effect of fiber diameter and orientation of fibroblast morphology and proliferation on electrospun poly(D,L-lactic-coglycolic acid) meshes. Biomaterials. 2006. 27. 5681-5688.

10. Bashur, C. Effect of electrospun mesh diameter, mesh alignment, and mechanical stretch on bone marrow stromal cells for ligament tissue engineering. Ph.D. Thesis. Virginia Polytechnic Institute. 2009.

11. Bini, T. et al. Electrospun poly(L-lactide-co- glycolide) biodegradable polymer nanofiber tubes for peripheral nerve regeneration. Nanotechnology. 15. 14591464. 2004.

12. Felten, D. and Jozefowicz, R. Netter's Atlas of Human Neuroscience, $2^{\text {nd }}$ edition. Saunders. 2009.

13. Hadlock, T., et. al. A Tissue-Engineered Conduit for Peripheral Nerve Repair. Arch otolaryngol head neck surg. 1998. 124.

14. Zanker, K. and Entschladen, F. Neuronal Activity in Tumor Tissue. S Karger Pub. 2007.

15. Butler, D. Mobilization of the Nervous System. Churchill Livingstone. 1991.

16. Sircar, S. Principles of Medical Physiology. Thieme. 2008.

17. Ratner, B. and Hoffman, A. Biomaterials Science. Academic Press. 2004.

18. Ramakrishna, S. An Introduction to Electrospinning and Nanofibers. World Scientific Publishing Company. (2005). 
19. Chu, P. and Liu, X. Biomaterials Fabrication and Processing Handbook. CRC Press. 2008.

20. Yoon, J. et al. Dexamethasone-releasing biodegradable polymer scaffolds fabricated by a gas-foaming/salt-leaching method. Biomaterials 2003. 24 2323-2329.

21. Laurencin, C. et al. Structural assessment of a tissue engineered scaffold for bone repair. Engineering in medicine and Biology Society, 2001. Proceedings of the 23rd Annual International Conference of the IEEE. 3. 2975-2978. 2001.

22. Zhang, S. Fabrication of novel biomaterials through molecular self-assembly. Nature Biotechnology. 21. 10. 1171-1178. 2003.

23. Gratzer, W. Giant Molecules. Oxford University Press. 2011.

24. Taylor, G. Disintegration of water drops in an electric field. Proceedings of the Royal Society of London. Series A. 280 (1382) 383-397. 1964.

25. Wang, H. et al. Creation of highly aligned electrospun poly-L-lactic acid fibers for nerve regeneration applications. Journal of Neural Engineering. 2009.

26. Lingaiah, S., Shivakumar, K., Sadler, R., Sharpe, M. Polyacrylonitrile-based Carbon Nanofibers Prepared by Electrospinning. SAMPE-06. Long Beach , CA. 2006.

27. Lee, K. et al. Polarized FT-IR Study of Macroscopically oriented Electrospun Nylon 6 Nanofibers. Macromolecules. 2008. 41. 1494-1498.

28. Jiang, $\mathrm{H}$. et al. A facile technique to prepare biodegradable coaxial electrospun nanofibers for controlled release of bioactive agents. Journal of Controlled Release. 2005. 108. 237-243.

29. Choi, J. et al. The influence of electrospun aligned poly( $\varepsilon$ caprolactone)/collagen nanofibers meshes on the formation of self-aligned skeletal muscle myotubes. Biomaterials. 2008.29. 2899-2906.

30. Matthews, J. et al. Electrospinning of Collagen Nanofibers. Biomacromolecules. 2002. 3. 232-238.

31. Tian, F. et al. Quantitative analysis of cell adhesion on aligned micro-and nanofibers. Journal of Biomedical Materials Research Part A. 2007. 291-299.

32. Deng, M. Novel Biocompatible Polymeric Blends for Bone Regeneration: Material and Matrix Design and Development. Ph.D. Thesis. University of Virginia. 2010.

33. Lakeshore Biomaterials. SurModics Pharmaceuticals. http://www.surmodicsbiomaterials.com/ 2009.

34. Frey, M. \& Li, L. Electrospinning and Porosity measurements of Nylon6/Poly(ethylene oxide) Blended Nonwovens. Journal of Engineered Fibers and Fabrics. 2007. 2. 1. 31-37.

35. Teo, W. et al. Porous tubular structures with controlled fiber orientation using a modified electrospinning method. Nanotechnology. 2005. 16. 918-924. 
36. Srivastava, Y. et al. Electrospinning of hollow and core/sheath nanofibers using a microfluidic manifold. Microfluid Nanofluid.2007. 4. 3. 245-250.

37. Sheng, X. \& Zhang, J. Superhydrophobic Behaviors of Polymeric Surfaces with Aligned Nanofibers. Langmuir. 2009. 25. 12. 6916-6922.

38. Kakade, M. et al. Electric Field Induced Orientation of Polymer Chains in Macroscopically Aligned Electrospun Polymer Nanofibers. Journal of American Chemical Society. 2007. 129. 2777-1782.

39. McClure, M. et al. Electrospinning-aligned and random polydioxanonepolycaprolactone-silk fibroin-blended scaffolds: geometry for a vascular matrix. Biomedical Materials. 2009. 4. 0550101.

40. Liu, L. \& Dzenis, Y. Analysis of the effects of the residual charge and gap size on electrospun nanofiber alignment in a gap method. Nanotechnology. 2008. 19.

41. Xie, J. et al. Aligned-to-random nanofiber scaffolds for mimicking the structure of the tendon-to-bone insertion site. Nanoscale. 2010. 2. 923-926.

42. Chung, S. et al. Nanofibrous scaffolds electrospun from elastomeric biodegradable poly(L-lactide-co- $\varepsilon$-caprolactone) copolymer. Biomedical Materials. 2009. 4.

43. Quin, X., Yang, E., Wang, S. Effect of Different Salts on Electrospinning of Polyacrylonitrile (PAN) Polymer Solution. Journal of Applied Polymer Science. 2006.3865-3870.

44. Hwang, N., Varghese, S. Elisseeff, J. Controlled differentiation of stem cells. Advanced Drug Delivery Reviews. 2008. 60. 199-214.

45. Corey, J. et al. Aligned electrospun nanofibers specify the direction of dorsal root ganglia neurite growth. Journal of Biomedical Materials Research Part A. 2006. 83. 3. 636-645.

46. Lee, J. H.; Jung, H. W.; Kang, I.-K.; Lee, H. B. Cell behaviour on polymer surfaces with different functional groups. Biomaterials 1994.15 (9). 705-711.

47. Liu, Y. et al. Magnetic-Field-Assited Electrospinning of Aligned Straight and Wavy Polymeric Nanofibers. Advanced Materials. 2010. 22. 2454-2457.

48. Yang, F. et al. Electrospinning of nano/micro scale poly(L-lactic acid) aligned fibers and their potential in neural tissue engineering. Biomaterials. 2005. 26. 2603-2610.

49. Lee, C. et al. Nanofiber alignment and direction of mechanical strain affect the ECM production of human ACL fibroblast. Biomaterials. 2005. 26. 1261-1270.

50. Wang, H. et al. Fabrication of aligned ferrite nanofibers by magnetic-fieldassisted electrospinning coupled with oxygen plasma treatment. Materials Research Bulletin. 2009. 44. 1676-1680. 
51. Li, D., Wang, Y., Xia, Y. Electrospinning Nanofibers as Uniaxially Aligned Arrays ad Layer-by Layer Stacked Films. Advanced Materials. 2004. 16. 4. 361-366.

52. Wang, J. et al. The effects of electrospun TSF nanofiber diameter and alignment on neuronal differentiation of human embryonic stem cells. Journal of Biomedical Materials Research Part A. 2012. 100A. 3. 632-645.

53. Mobarakeh, L. et al. Electrospun Poly ( $\varepsilon$-caprolactone) nanofiber Mat as Extracellular Matrix. Yakhteh Medical Journal. 2008. 10. 3. 179-184.

54. Lowery, J., Datta, N., Rutledge, G. Effect of fiber diameter, pre size and seeding method on growth of human dermal fibroblasts in electrospun poly $(\varepsilon-$ caprolactone) fibrous mats. Biomaterials. 2010. 31. 491-504.

55. Selhuber-Unkel, C.; Erdmann, T.; Lopez-Garcia, M.; Kessler, H.; Schwarz, U. S.; Spatz, J. P. Cell Adhesion Strength Is Controlled by Intermolecular Spacing of Adhesion Receptors. Biophysical Journal 2010. 98. 543-551.

56. Carnell, L., et al. Aligned Mats from Electrospun Single Fibers. Macromolecules. 2008. 41. 5345-5349.

57. Badami, A., et al. Effect of fiber diameter on spreading, proliferation, and differentiation of osteoblastic cells on electrospun poly(lactic acid) substrates. Biomaterials. 2006. 27. 596-606.

58. Zhu, Y., et al. Macro-Alignment of Electrospun Fibers For Vascular Tissue Engineering. Journal of Biomedical Materials Research Part B. 2009. 508516.

59. Sarkar, S., et al. Biased AC Electrospinning of Aligned Polymer Nanofibers. Macromolecules Rapid Communication 2007. 28. 1034-1039.

60. Quiles, M., et al. Highly polarized luminescence from aligned conjugated polymer electrospun nanofibers. Applied Physics Letters. 2008. 92.

61. Acharya, M., et al. Dual Electric Field Induced Alignment of Electrospun Nanofibers. Macromolecular Materials and Engineering. 2008. 293. 666-674.

62. Yan, H., Liu, L., Zhang, Z. Alignment of electrospun nanofibers using dielectric materials. Applied Physics Letters. 2009. 95. 14.

63. Chaurey, V.; Chiang, P.-C.; Polanco, C.; Su, Y.-H.; Chou, C.-F.; Swami, N. S. Interplay of Electrical Forces for Alignment of Sub-100 nm Electrospun Nanofibers on Insulator Gap Collectors. Langmuir. 2010. 26 (24). 1902219026.

64. Lanza, R. Principles of Tissue Engineering. Academic Press. 2007.

65. Xiong, L., Jiang, H., Wang, D. Synthesis, characterization and degradation of poly (D,L-lactide) block polyvinylpyrrolidone-block-poly(D,L-lactide) copolymers. Journal of Polymer Research. 2009. 16. 191-197. 
66. Risbud, M. \& Bhat, S. Properties of polyvinyl pyrrolidone / $\beta$-chitosan hydrogel membranes and their biocompatibility evaluation by haemorheological method. Journal of Material Science: Materials in Medicine. 2001. 12. 75-79.

67. Wen, X., Tresco, P. Fabrication and characterization of permeable degradable poly poly(DL-lactide-co-glycolide) (PLGA) hollow fiber phase inversion membranes for use as nerve tract guidance channels. Biomaterials. 2006. 27. 3800-3809.

68. Huh, K. et al. PIGA-PEG Block Copolymers for Drug Formulations. Drug Delivery Technology. 3. 5. 2003.

69. Aviss, K., et al. Aligned Electrospun Polymer Fibers for Skeletal Muscle Regeneration. European Cells and Materials. 2009. 19. 193-204.

70. Jose, M. et al. Aligned PLGA/HA nanofibrous nanocomposite scaffolds for bone tissue engineering. Acta Biomaterialia.2009. 5. 305-315.

71. Bini, T. et al. Poly(I-lactide-co-glycolide) biodegradable microfibers and electrospun nanofibers for nerve tissue engineering: an in vitro study. $J$ Material Science. 2006. 41. 6453-6459.

72. Shang, S. The effect of electrospun fiber alignment on the behavior of rat periodontal ligament cells. European Cells and Materials. 2010. 19. 180-192.

73. Deitzel, J., et al. The effect of processing variables on the morphology of electrospun nanofibers and textiles. Polymer. 2001. 42. 261-272.

74. Pham, Q., et al. Electrospinning of Polymeric Nanofibers for Tissue Engineering Applications. Tissue Engineering. 2006. 12. 5.

75. Zhong, S., et al. An aligned nanofibrous collagen scaffold by electrospinning and its effects on in vitro fibroblast culture. Journal of Biomedical Materials Research Part A. 2006. 456-463.

76. Huang, J., et al. Quantifying the Effects of Molecular Orientation and Length on Two-dimensional Receptor-Ligand Binding Kinetics. Journal of Biological Chemistry. 2004. 279. 43. 44915-44923.

77. Kim, S., et al. Highly Oriented and Ordered Arrays from Block Copolymers via Solvent Evaporation. Advanced Materials. 2004. 16. 3.

78. Kimura, N., et al. Molecular Orientation and Crystaline Structure of Aligned Electrospun Nylon-6 Nanofibers: Effect of Gap Size. Macromolucular Material Engineering. 2010. 295. 1090-1096.

79. Chan, K., et al. Effect of Molecular Orientation on Mechanical Property of Single Electrospun Fiber of Poly[ ${ }^{\circledR}-3-h y d r o x y b u t y r a t e-c o-\circledR-3-$ hydroxyvalerate]. Journal of Physical Chemistry Part B. 2009. 113. 1317913185.

80. Yee, W., et al. Morphology, polymorphism behavior and molecular orientation of electrospun poly(vinylidene fluoride) fibers. Polymer. 2006. 48. 512-521. 
81. Teo, W. and Ramakrishna, S. A review on electrospinning design and nanofiber assemblies. Nanotechnology. 2006. 17. R89-R106.

82. Jouyban, A., et al. A simple relationship between dielectric constant of mixed solvents with solvent composition and temperature. International Journal of Pharmaceutics. 2004. 2. 28. 353-360.

83. Baca, A. and Thelen, D. "Electrospinning Process and Apparatus for Aligned Fiber Production.” US Patent \# 20110018174. January 27, 2011.

84. Melikyan, G. and Deravakian, A. Tetrahydrofuran as an one-electron donor. Journal of Organometallic Chemistry. 1997. 544. 1. 143-145.

85. Son, W., et al. The effects of solution properties and polyelectrolyte on electrospinning of ultrafine poly(ethylene oxide) fibers. Polymer. 2004. 45. 2959-2966.

86. Maheshwari, S. and Chang, H. Assembly of Multi-stranded Nanofiber Threads through AC Electrospinning. Advanced Materials. 2009. 21. 349-354.

87. Yang, D., et al. Fabrication of Aligned Fibrous Arrays by Magnetic Electrospinning. Advanced Materials. 2007. 19. 3702-3706.

88. Hurtado, A., et al. Poly (D,L-lactic acid) macroporous guidance scaffolds seeded with Schwann cells genetically modified to secrete a bi-functional neurotrophin implanted in the completely transected adult rat thoracic spinal cord. Biomaterials. 2006. 27. 430-442.

89. Hadlock, T., et al. A Polymer Foam Conduit Seeded with Schwann Cells Promotes Guided Peripheral Nerve Regeneration. Tissue Engineering. 2000. 6. 2.

90. Wen, X. and Tresco, P. Effect of filament diameter and extracellular matrix molecule precoating on neurite outgrowth and Schwann cell behavior on multifilament entubulation bridging device in vitro. Journal of Biomedical Materials Research Part A. 2006. 1. 76. 3. 626-637.

91. Subramanian, A., et al. Fabrication of uniaxially aligned 3D electrospun scaffolds for neural regeneration. Biomedical Materials.2011.

92. Gupta, D., et al. Aligned and random nanofibrous substrate for the in vitro culture of Schwann cells for neural tissue engineering. Acta Biomaterialia. 2009. 2560-2569.

93. Resende, V., et al. Strategies for inducing the formation of bands of Büngner in peripheral nerve regeneration. Biomaterials. 2009. 5251-5259.

94. Stocum, D. Regenerative Biology and Medicine. Academic Press. 2006.

95. Foley, J., et al. Cooperative modulation of neuritogenesis by PC12 cells by topography and nerve growth factor. Biomaterials. 2005. 3639-3644.

96. Lee, J., et al. Polypyrrole-Coated Electrospun PLGA Nanofibers for Neural Tissue Applications. Biomaterials. 2009. 30. 26. 4325-4335. 
97. Cooper, A., et al. Fabrication and cellular compatibility of aligned chitosanPCL fibers for nerve tissue regeneration. Carbohydrate Polymers. 2011. 85. 149-156.

98. Sitterley, G. Poly-L-Lysine Cell Attachment Protocol. Biofiles. 2008. 3. 8. 12. 99. Wang, Y., et al. A Novel Controlled release Drug Delivery System for Multiple Drugs based on Electrospun Nanofibers Containing Nanoparticles. Journal of Pharmaceutical Sciences. 2010. 99. 19.

100. Qi, X., et. al. Sustained delivery of sphingosine-1-phosphate using poly(lactic-co-glycolic acid)-based microparticles stimulates Akt/ERK-eNOS mediated angiogenesis and vascular maturation restoring blood flow in ischemic limbs of mice. European Journal of Pharmacology. 634. 2010. 121131.

101. Sefick, L., et. al. Sustained release of sphingosine 1-phosphate for therapeutic arteriogenesis and bone tissue engineering. Biomaterials. 2008. 29.

102. Zolnik, B. and Burgess, D. Effect of acidic pH on PLGA microsphere degradation and release. Journal of Controlled Release. 2007. 122. 338-344.

103. Moghe, A. and Gupta, B. Co-axial Electrospinnig for Nanofiber Structure: Preparation and Applications. Polymer Reviews. 2008. 48. 353-377.

104. Ranganath, S. and Wang, C. Local intracranial drug delivery using biodegradable PLGA-paclitaxel micro/nanofiber implants to treat malignant brain tumors. AlChE conference proceedings. 2008.

105. Chakraborty, S., et al. Electrohydrodynamics: A facile technique to fabricate drug delivery systems. Advanced Drug Delivery Reviews. 2009. 61. 1043-1054.

106. Han, D., et al. Versatile Core-Sheath Biofibers using Coaxial Electrospinning. Material Research Society Symposiam Proceedings Volume 1094. 2008.

107. Wang, C., et al. Biodegradable Core/Shell Fibers by Coaxial Electrospinning. Macromolecules. 2010. 43. 6389-6397.

108. Kim, G., et al. Coaxially electrospun micro/nanofibrous poly( $\varepsilon-$ caprolactone)/eggshell-protein scaffold. Bioinspiration \& Biomimetics. 2008. 3.

109. Li, M., et al. Co-electrospun poly(lactide-co-glycolide), gelatin, and elastin blends for tissue engineering scaffolds. Journal of Biomedical Materials Research Part A.2006. 963-973.

110. Zhang, H., et al. Electrospinning of ultrafine core/shell fibers for biomedical applications. Science China. 2010. 6. 1246-1254.

111. Leung, V. and Ko, F. Biomedical applications of nanofibers. Polymers advanced technologies. 2010. 
112. Hwang, C., et al. Microfluidic Chip-based fabrication of PLGA microfiber scaffolds for Tissue Engieering. Langmuir. 2008. 24. 6845-6851.

113. Zebco. America's Favorite Fishing Rods. www.zebco.com. 2011. 


\section{Appendix A: Guide to Polymers and Solvents}

Solvents for polymers are listed in the table below:

\begin{tabular}{lc}
\hline \multicolumn{1}{c}{ Solvent name } & Molecular Formula \\
\hline Water & $\mathrm{H}_{2} \mathrm{O}$ \\
Dichloromethane (DCM or methylene & $\mathrm{CH}_{2} \mathrm{Cl}_{2}$ \\
chloride) & \\
Chloroform & $\mathrm{CHCl}_{3}$ \\
Dimethylformamide (DMF) & $\mathrm{C}_{3} \mathrm{H}_{7} \mathrm{NO}$ \\
Tetrahydrofuran (THF) & $\mathrm{C}_{4} \mathrm{H}_{8} \mathrm{O}$ \\
Dimethyl sulfoxide (DMSO) & $\mathrm{C}_{2} \mathrm{H}_{6} \mathrm{OS}$ \\
Methanol (MetOH) & $\mathrm{CH}_{3} \mathrm{OH}$ \\
Ethanol (EtOH) & $\mathrm{C}_{2} \mathrm{H}_{5} \mathrm{OH}$ \\
Formic acid & $\mathrm{CH}_{2} \mathrm{O}_{2}$ \\
Hexafluoroisopropanol (HFIP) & $\mathrm{C}_{3} \mathrm{H}_{2} \mathrm{~F}_{6} \mathrm{O}$ \\
\hline
\end{tabular}

There are a wide variety of different biocompatible polymers used in tissue engineering. Some of the more common polymers are listed in the following table. These polymers are dissolved in a wide variety of various solvents, ranging from water to Dimethyl sulfoxide (DMSO).

Polymer concentration is typically expressed in the form of a weight (grams) over volume (milliliters or $\mathrm{mL}$ ) ${ }^{*} 100 \%$. For example, $200 \mathrm{mg}$ of PLGA dissolved in $1 \mathrm{~mL}$ of HFIP is $20 \%$ concentration.

$$
\frac{\text { grams }}{\text { milliliters }} * 100 \%=\text { concentration }
$$




\begin{tabular}{|c|c|c|c|c|c|}
\hline & $\begin{array}{c}\text { Full polymer } \\
\text { name }\end{array}$ & Chemical structure & $\begin{array}{c}\text { Solvents/typic } \\
\text { al } \\
\text { concentration } \\
s \\
\end{array}$ & $\begin{array}{c}\text { Unique } \\
\text { attributes }\end{array}$ & References \\
\hline $\begin{array}{c}\text { Nylon- } \\
6\end{array}$ & polycaprolactam & & formic acid & $\begin{array}{c}\text { Structure is } \\
\text { similar to } \beta \text { sheets } \\
\text { of natural } \\
\text { polypeptides }\end{array}$ & 11 \\
\hline PAN & $\begin{array}{c}\text { polyacrylonitrile } \\
\text { (acrylic) }\end{array}$ & & DMF $10-20 \%$ & Non-degradable & 15,30 \\
\hline PCL & polycaprolactone & & $\begin{array}{c}\text { DCM/MetOH } \\
\text { HFIP } \\
\text { Chloroform } \\
\text { THF } \\
\text { THF:DMF } \\
10-30 \%\end{array}$ & $\begin{array}{c}\text { FDA approved } \\
\text { Long degradation } \\
\text { times }\end{array}$ & $\begin{array}{c}14,18,24,28 \\
61,64\end{array}$ \\
\hline PEG & $\begin{array}{c}\text { Polyethylene } \\
\text { glycol }\end{array}$ & & Water & $\begin{array}{l}\text { Water soluble } \\
\text { Molecular mass } \\
<20000 \mathrm{~g} / \mathrm{mol}\end{array}$ & 13,17 \\
\hline PEO & $\begin{array}{c}\text { Polyethylene } \\
\text { oxide }\end{array}$ & & $\begin{array}{c}\text { EtOH } \\
\text { Water } \\
\text { Chloroform } \\
\text { DMF/Water }\end{array}$ & $\begin{array}{l}\text { Water soluble } \\
\text { conductive } \\
\text { Molecular mass } \\
>20000 \mathrm{~g} / \mathrm{mol}\end{array}$ & 56 \\
\hline PGA & $\begin{array}{l}\text { Polyglycolide or } \\
\text { Poly(glycolic } \\
\text { acid) }\end{array}$ & 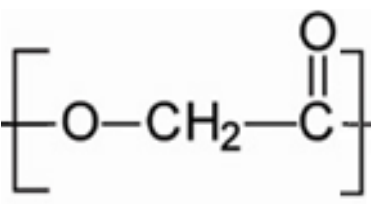 & $\begin{array}{c}\text { DCM } \\
\text { HFIP } \\
\text { Chloroform }\end{array}$ & $\begin{array}{c}\text { Short degradation } \\
\text { time } \\
\text { Degrades into } \\
\text { lactic acid }\end{array}$ & 20,24 \\
\hline
\end{tabular}




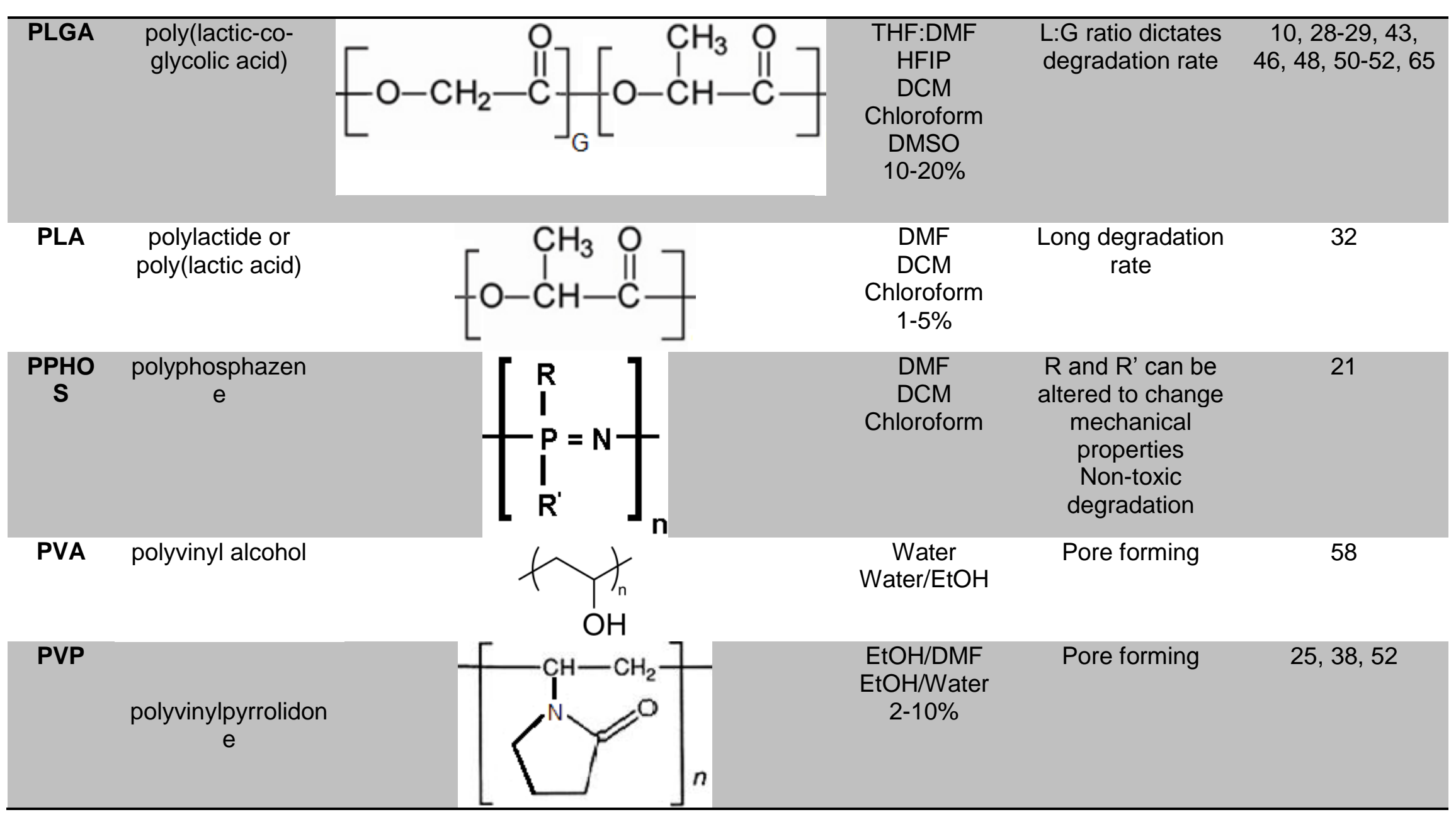




\section{Appendix B: Further information on electrospinning techniques}

\section{Section B.1 Nanofiber areas of best alignment}

When electrospinning nanofibers, our model shows that the stretching force, $E_{x}$, is strongest at the edge of the collector. Typically, nanofibers at the edges of collectors are poorly aligned. Nanofiber alignment is always best at the center of the patterned Mylar ${ }^{\mathrm{TM}}$ collector. This is because the residual charge responsible for aligning nanofibers is dominant at the center of each collector. Nanofiber collection is depicted in Figure B.1
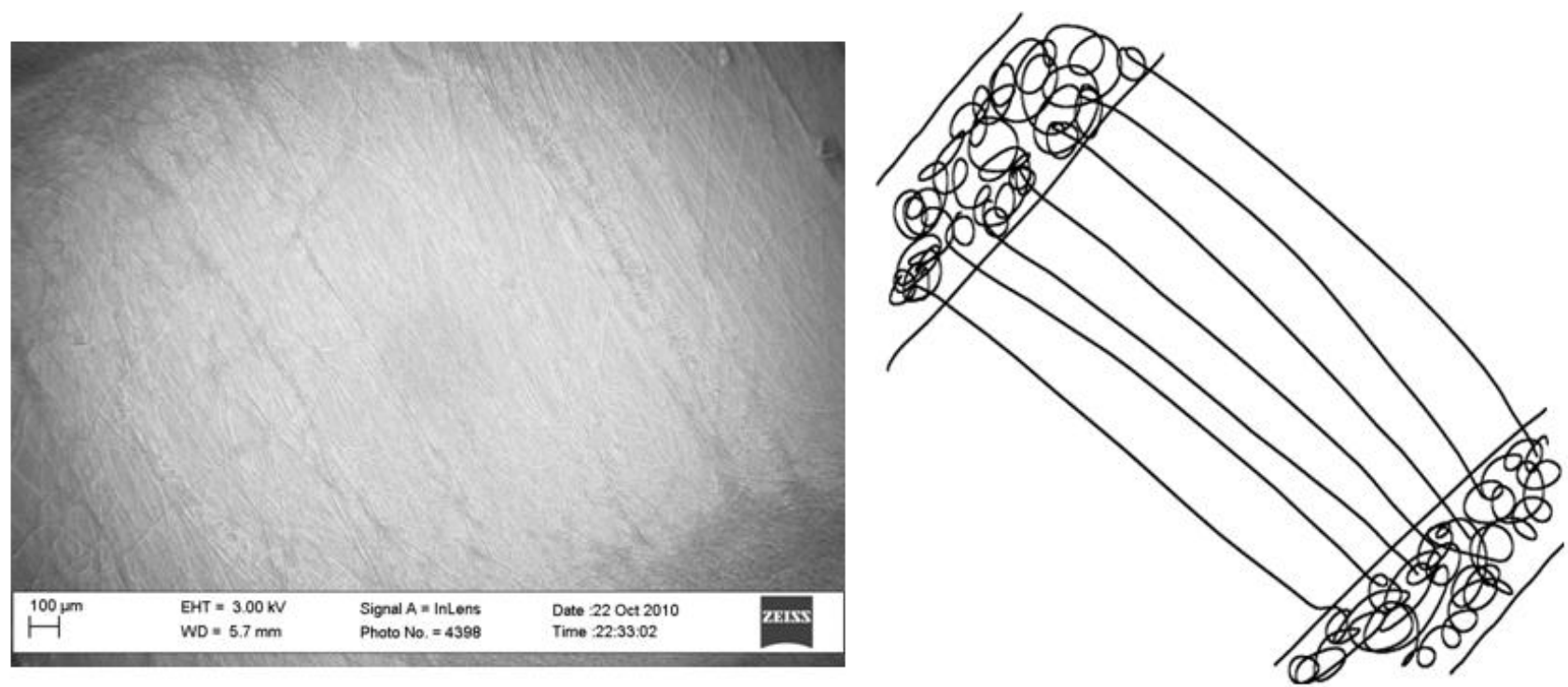

Figure B.1: Low magnification image and cartoon of nanofibers collecting on a dielectric gap. Note the aligned fibers in the center of the weak dielectric area and random fibers in the area with a higher dielectric.

In mandrel electrospinning, the area of best alignment is dependent on the distance from the central area of maximum nanofiber density. Typically the most aligned 
nanofibers are located a few centimeters away from densely collected nanofibers. This is depicted in Figure B.2

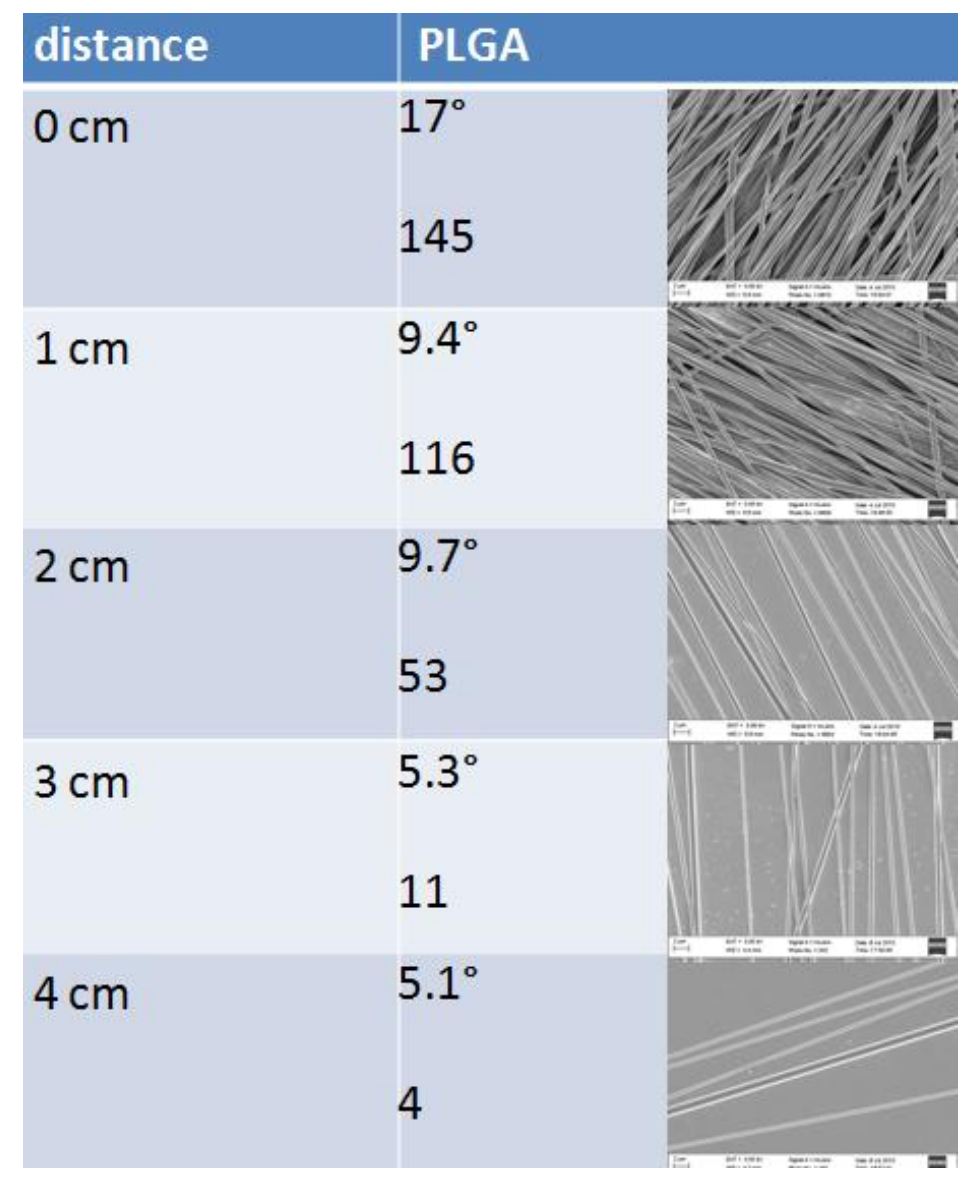

Figure B.2: fiber angular deviation and collection density as a function of distance from ground on a rotating mandrel. The bottom right number is fiber density. It is reported as the number of fibers per unit length of 35.9 microns.

Figure B.2 shows that it is very difficult to capture ideal collection density, which lies between 1 and 2 centimeters from ground. This collection density is ideal because the entire substrate is covered with well-aligned nanofibers. The density is also high enough to allow the fiber mesh to be removed from the mylar film for future applications. One hypothesis for why this spot $1.5 \mathrm{~cm}$ from the ground provides best fiber density and 
aligned collection is that the electrospinning cone of fibers is oriented perpendicularly or anti-perpendicularly to the rotating mandrel at this point.

\section{Section B.2 Fiber collection density}

Nanofiber collection density is a very difficult property to control. Time is the most crucial factor in determining fiber density. As Figure B.3 shows, density can be improved without decreasing alignment by utilizing a patterned mandrel. This phenomenon does not occur in other methods of nanofiber collection.

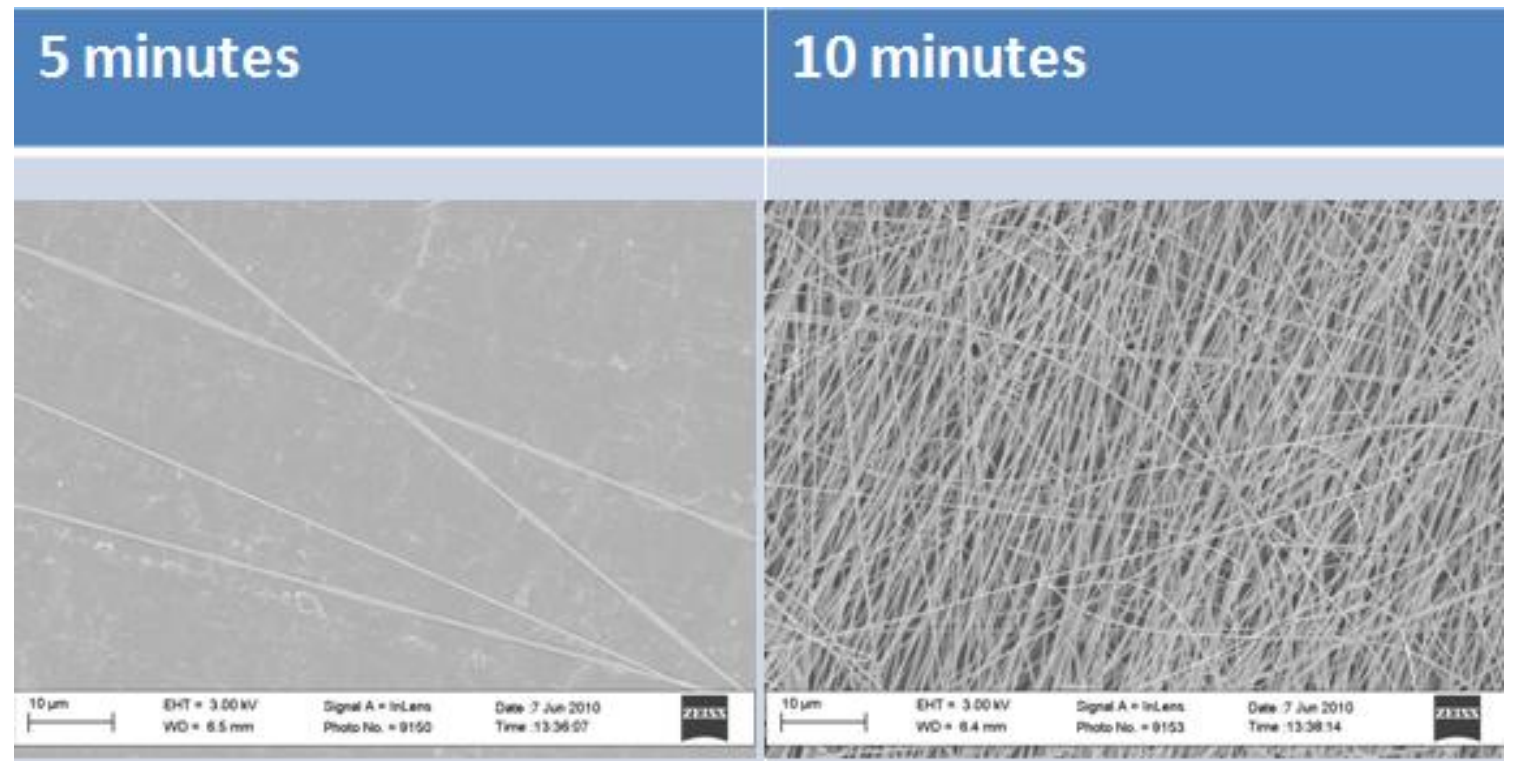

Figure B.3: time difference in nanofiber collection on mandrel. Note the drastic differences in density and alignment. 5 minutes yields low density and good alignment while 10 minutes gives dense misaligned nanofibers with $A D>30^{\circ}$.

Fiber density is highly variable across a single run. It is also interesting to note that fiber alignment is dependent upon the distance from ground. As fibers become less dense their angular deviation actually improves. Once too many fibers are deposited in a 
single area, the shear force of the mandrel is distorted from the retained positive charges in the thick nanofiber mesh.

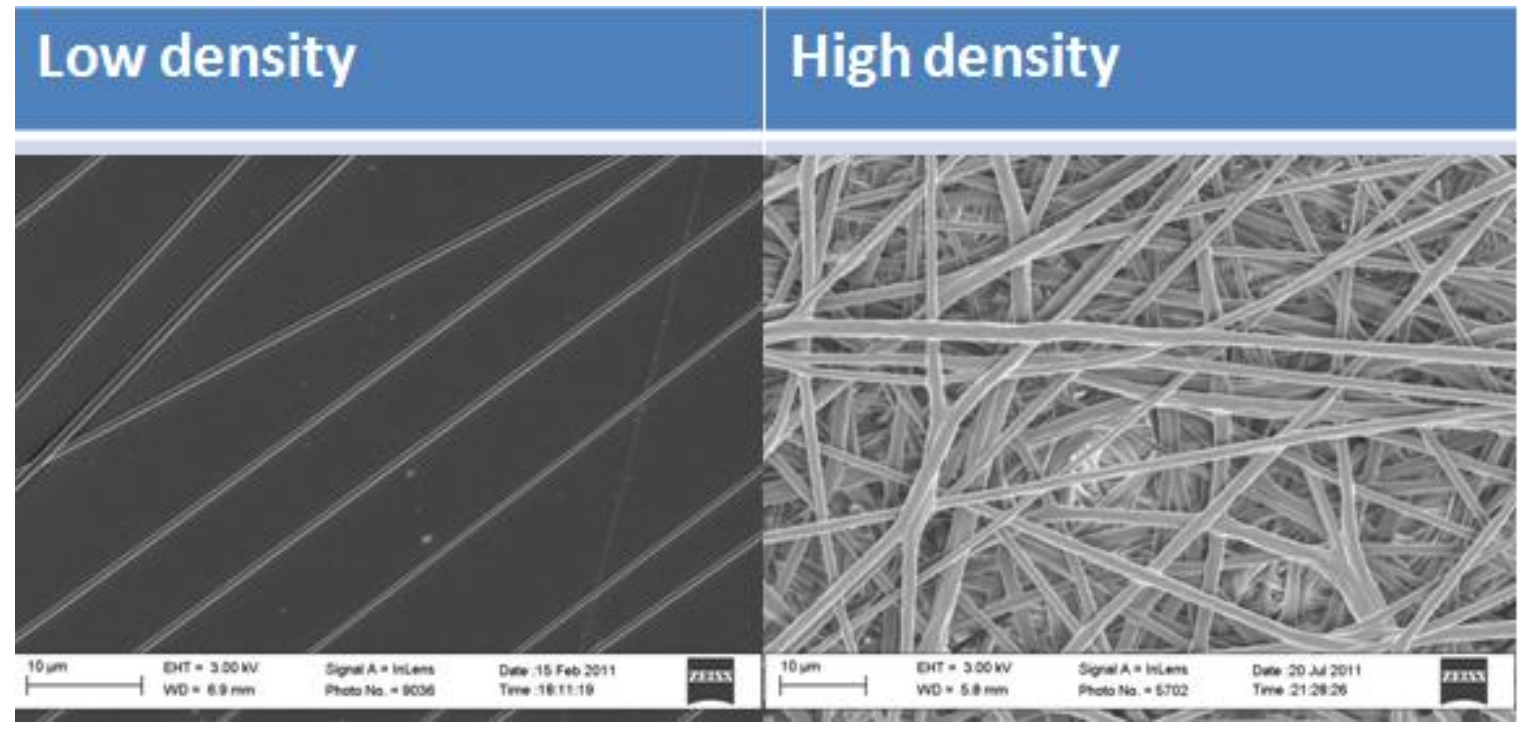

Figure B.4: high and low collection density on $4 \mathrm{~mm}$ Mylar ${ }^{\mathrm{TM}}$ patterned plate collectors. Fibers at low density were collected for 5 minutes and have good angular deviation, whereas high density fibers were collected for 10 minutes and are considered misaligned with $A D>30^{\circ}$.

From Figure B.4, it is apparent that fibers do not retain their alignment at high mesh thickness on plate collectors. A collection time between 5 and 10 minutes is required to form nanofibers with acceptable coverage and angular deviation. A major advantage of dielectric block and patterned mandrel techniques is thicker meshes can be created with good alignment. 


\section{Appendix C: Schwann cell culture, seeding, and staining procedures}

Schwann cells media

- 445 (500-55) ml DMEM

- $\quad 50 \mathrm{~mL}$ Fetal Bovine Serum - 10\% v/v

- $5 \mathrm{ml}$ Antibiotic/Antimycotic or pen-strp $-1 \% \mathrm{v} / \mathrm{v}$

\section{Splitting Cells}

1. Turn on water bath

a. Put in media, trypsin, PBS

2. Clean hood with ethanol and wipe

3. Take 2 new cell culture dishes out of pack and put in hood

a. Label dishes with cell type, passage, date, seeding density, and your name

4. Once liquids are warm, spray the bottles with $70 \%$ ethanol and wipe before putting in hood

a. Take cells out and check under microscope

5. Remove media (glass pipette)

6. Wash with PBS (5-6mL)

7. Remove PBS (new glass pipette).(wash/remove PBS twice) Save the glass pipette at this step

8. Add $1.5 \mathrm{~mL}$ Trypsin to each dish (large dishes)(make sure Tryspin covers all area of the dish) and wait for $30 \mathrm{sec}$ at room temp. and remove Trypsin.

9. Wait for 30-60 secs and tap sides to encourage cells to leave dish

10. Using a fresh pipette $(10 \mathrm{ml})$, add $10 \mathrm{ml}$ media to each new dish and $5 \mathrm{~mL}$ media to old dish

11. Aspirate cells to break up clumps (use pipette aid, at fast setting)

12. Add $0.5 \mathrm{~mL}$ to a new dish and $0.25 \mathrm{~mL}$ to another dish

13. Check the cells under microscope for proper density and no clumps and put the new dishes in incubator

14. Remove the remaining cell suspension from the old dishes (using the glass pipette). Bleach the dishes before throwing them in biohazard waste box.

15. Put all the bottles back in refrigerator.

16. Spray and clean the hood with cavicide

Cell seeding process may begin at Step 11. Cell seeding density is 2500 cells/cm2. Fix with f-actin stain after 24 hours. 


\section{F-actin stain procedure}

Materials:

- $4 \%$ PFA - 2 grams PFA in 50mL 1 X PBS with 100uL NaOH - dissolve 8 hours

- $.1 \%$ tritonX 100/PFA - 15uL triton in 15mL PFA solution

- $1 \%$ BSA in PFA - 200mg BSA in 20mL PBS

- Stain antibody 1:40 dilution - 400mL BSA solution + 400mL PBS

Procedure:

Fix with PFA - 15min

Wash with PBS once

Triton $X-10 \min$

Wash with PBS $3 \mathrm{X}$

$\mathrm{BSA}-15 \mathrm{~min}$

Wash with PBS $3 \mathrm{X}$

100uL drop of stain on parafilm turn sample upside down to soak- $15 \mathrm{~min}$

Wash with PBS $3 \mathrm{X}$

Take images. Dye becomes inactive after 6 hours. 


\section{Appendix D: Cellular SEM preparation procedure}

1. Fix cells with glutaraldehyde for 6 hours or overnight at room temperature

2. Dehydration wash:
a. 15 minutes $25 \% \mathrm{EtOH}$
b. 15 minutes $50 \% \mathrm{EtOH}$
c. 15 minutes $75 \% \mathrm{EtOH}$
d. 15 minutes $85 \% \mathrm{EtOH}$
e. 15 minutes $95 \% \mathrm{EtOH}$
f. 15 minutes $100 \% \mathrm{EtOH}$

3. Allow samples to dry overnight at room temperature

4. Mount samples on to SEM studs using carbon tape

5. Coat with $12 \mathrm{~nm}$ of gold/palladium

6. Take images 


\section{Appendix E: PC12 cell maintenance and seeding procedures}

PC 12 media formula

- $407.5(500-92.5) \mathrm{mL}$ of DMEM/F12(1:1)

- $5 \mathrm{~mL}$ Pen Strep - 1\% v/v

- $75 \mathrm{~mL}$ Horse Serum - 15\% v/v

- $12.5 \mathrm{~mL}$ Fetal Bovine Serum - 2.5\% v/v

Splitting Cells

1. Turn on water bath

a. Put in media

2. Clean hood with ethanol and wipe

3. Take 2 new cell culture dishes out of pack and put in hood

a. LABEL DISHES! (cell type, passage, date, seeding density and your name)

4. Once liquids are warm, spray the bottles with $70 \%$ ethanol and wipe before putting in hood

a. Take cells out and check under microscope

5. Remove media from the cell dish (use glass Pasteur pipette). Save the pipette at this step to be used in step 14. If you need a glass pipette before step 14, use a new one.

6. Using a new pipette $(10 \mathrm{ml})$ Add $5-7 \mathrm{ml}$ of media to each of the new dishes

7. Add $2 \mathrm{ml}$ of fresh media (volume can be changed depending on split ratio, eg. $2 \mathrm{ml}$ for $1: 2,3 \mathrm{ml}$ for $1: 3$ etc.) to the cells (old dish)

8. Use cell scraper to gently scrape cells towards you 
9. Rotate dish 180 degrees

10. Use cell scraper to gently scrape cells towards you

11. Aspirate cells to break up clumps (use pipette aid the fast setting)

12. Add $1 \mathrm{~mL}$ of the cell suspension to new dish

13. Check the cells under microscope for proper density and no clumps and put the new dishes in incubator

14. Remove the remaining cell suspension from the old dishes (using the glass pipette). Bleach the dishes before throwing them in biohazard waste box.

15. Put all the bottles back in refrigerator.

16. Spray and clean the hood with cavicide

Cells may be seeded on samples at step 11 . Seeding density is $2.5 \times 10^{4}$ cells $/ \mathrm{cm}^{2}$

Cells require $50 \mathrm{ng} / \mathrm{mL}$ NGF 24 hours after seeding. Image cells on day 5 by adding $1 \mu \mathrm{g} / \mathrm{mL}$ Calcein AM as a fluorescent stain.

Changing Media

1. Turn on water bath

a. Put media in

2. Clean hood with ethanol and wipe

3. Once liquids are warm, spray the bottles with $70 \%$ ethanol and wipe before putting in hood

a. Take cells out and check under microscope

4. Remove media from the cell dish ( use glass Pasteur pipette)

5. Using a new pipette $(10 \mathrm{ml})$ add $5-7 \mathrm{ml}$ of fresh media to the cells

6. Put all the bottles back in refrigerator.

7. Spray and clean the hood with cavicide 